\title{
A Viscous Fluid Flow through a Thin Channel with Mixed Rigid-Elastic Boundary: Variational and Asymptotic Analysis
}

\author{
R. Fares, ${ }^{1}$ G. P. Panasenko, ${ }^{1}$ and R. Stavre ${ }^{2}$ \\ ${ }^{1}$ Institut Camille Jordan UMR, CNRS 5208, PRES University of Lyon/University of Saint-Etienne, \\ 23 Rue Paul Michelon, 42023 Saint-Etienne, France \\ 2 Institute of Mathematics "Simion Stoilow", Romanian Academy, P.O. Box 1-764, \\ 014700 Bucharest, Romania
}

Correspondence should be addressed to G. P. Panasenko, grigory.panasenko@univ-st-etienne.fr

Received 23 January 2012; Accepted 29 February 2012

Academic Editor: D. O’Regan

Copyright (C) 2012 R. Fares et al. This is an open access article distributed under the Creative Commons Attribution License, which permits unrestricted use, distribution, and reproduction in any medium, provided the original work is properly cited.

\begin{abstract}
We study the nonsteady Stokes flow in a thin tube structure composed by two thin rectangles with lateral elastic boundaries which are connected by a domain with rigid boundaries. After a variational approach of the problem which gives us existence, uniqueness, regularity results, and some a priori estimates, we construct an asymptotic solution. The existence of a junction region between the two rectangles imposes to consider, as part of the asymptotic solution, some boundary layer correctors that correspond to this region. We present and solve the problems for all the terms of the asymptotic expansion. For two different cases, we describe the order of steps of the algorithm of solving the problem and we construct the main term of the asymptotic expansion. By means of the a priori estimates, we justify our asymptotic construction, by obtaining a small error between the exact and the asymptotic solutions.
\end{abstract}

\section{Introduction}

A few years ago we began to publish several papers dealing with an asymptotic analysis for an interaction problem between a viscous fluid and an elastic structure. Problems involving the interaction between a fluid and a deformable structure have been studied extensively in the last years due to their applications in many areas such as: engineering, biomechanics, biology, and hydroelasticity.

In order to model the blood flow through a vessel we considered in [1] a non steady viscous flow in a thin rectangle with elastic walls, when at the ends of the flow domain periodicity conditions are imposed. The asymptotic approach was then extended for 
the nonperiodic case in [2], when the inflow and outflow velocities are given functions. Generalizations of the previous cited papers were obtained in [3-5]. In the first two papers we extended the results of [1] to a-three dimensional case, by considering a periodic, axisymmetric flow in a thin cylinder with elastic lateral boundary, while in [4] we studied two different cases: when the inertial term of the equation for the wall displacement is much smaller than the stress term and when these two terms are of the same order. Moreover, [5] deals with the asymptotic analysis for a fluid with variable viscosity.

In all these papers, the fluid flow was described by the Stokes equations and the behavior of the elastic structure was simulated either by the Sophie Germain's or by the Koiter's equation. The fluid-structure interaction was mathematically expressed by the equality of the fluid velocity at the boundary and the time derivative of the wall displacement. We constructed an asymptotic solution and we proved that it represents a good approximation for the exact solution, by obtaining a small error between them.

In this paper we consider the nonsteady Stokes flow in a thin tube structure. In twodimensional case, a tube structure is some connected union of thin rectangles, having a dimension much smaller than the other one. The main difference between this paper and our previous works $[2,5]$ is that now the boundary of the flow domain contains elastic parts and rigid parts, as well.

The flow domain consists of two thin rectangles with elastic boundary. The junction between the rectangles is realized by means of rigid boundaries. The interaction between the viscous fluid and the elastic boundaries produces normal displacements. The elastic boundaries behavior is described by the Sophie Germain's equation.

This domain models a vessel structure where a stent was placed. In fact, for treating arterial stenoses or occlusions, percutaneous angioplasty is indicated. It uses small inflatable balloons, single-use, that help dilate the artery at the site of narrowing. During angioplasty, a small wire mesh tube called a stent may be permanently placed in the newly opened artery or vein to help it remain open.

There are two types of stents: bare stents (wire mesh) and covered stents (also commonly called stent grafts). The first are in use as part of the expansion of retrecissements vessels, the second impermeable and can be used to prevent or treat hemorrhage. Stents are used for arteries in the heart, the kidney, the arm, or the leg. They are also used for the aorta in the abdomen or chest (see Figures 1 and 2).

We assimilate then the part of the vessel where the stent is placed to a domain with rigid boundaries.

We suppose that the viscous fluid has a variable viscosity depending on a longitudinal variable for each rectangle. This situation models a blood flow in a vessel structure where the viscosity depends on the concentration of some substances diluted in blood or some blood cells. Indeed, the asymptotic analysis of the convection-diffusion equation set in such domains $[6,7]$ shows that in the case of the Neumann (impermeability) condition at the lateral boundary and small Reynolds numbers, the concentration is asymptotically close to the one-dimensional description, that is, the convection-diffusion equation set on the graph. The solution of the problem on the graph is the leading term of the asymptotic expansion, and it evidently depends on the longitudinal variable. On the other hand, the viscosity often depends on the concentration of the diluted substances or distributed cells, and so, it depends on the longitudinal variable. Of course, the fluid motion equation is coupled with the diffusion-convection equation in this case. However, if the velocity is small (in our case, it is of order $\varepsilon^{2}$ ), then neglecting the convection, in comparison with the diffusion term or iterating with respect to the small term,we get the steady state diffusion equation; in absence of 


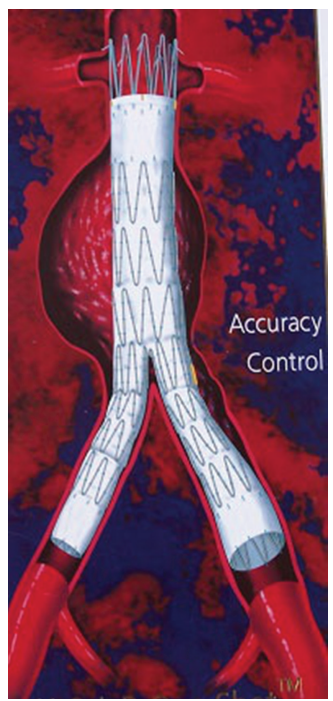

Figure 1: Bifurcation stenting.

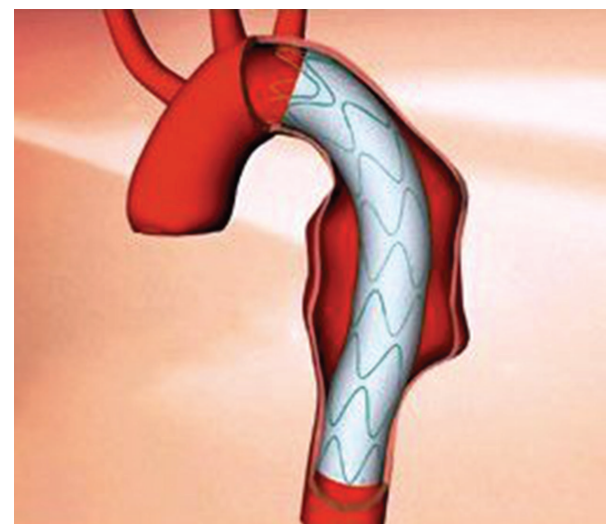

Figure 2: Stenting.

the source term in the right-hand side, it has a piecewise-linear asymptotic solution on the graph for the concentration. So, in this simplified situation, the diffusion equation can be solved before the fluid motion equation, and we obtain for the flow, the Stokes or NavierStokes equation with a variable viscosity depending (via concentration) on the longitudinal variable.

There are, of course, many other practical problems involving fluids with variable viscosity. For example, the presence of bacteria in suspension (see [8]) may change locally the viscosity.

The outline of the paper is as follows. In Section 2 we give a description of the flow domain and of the coupled system which models our problem. The next section presents the variational formulation of the problem. The literature contains an important number of papers dealing with a variational approach of fluid-structure interaction problems. For instance, results concerning the existence of weak or strong solutions when the fluid domain is 
either fixed or depending on time can be found in [9-12]. We establish in this section results such as: existence, uniqueness, regularity and a priori estimates. In Section 4 we construct the asymptotic solution. As in our previous papers [1-5], the problem depends on two small parameters. The first small parameter, $\varepsilon$, is defined as the ratio of the dimensions of the thin rectangles; the second one, $\delta$, corresponds to the softness of the wall. For various values of the small parameters $\varepsilon$ and $\delta$, an asymptotic expansion of the solution is constructed; the parameter $\delta$ is taken of the form $\delta=\varepsilon^{\gamma}$, with $\gamma \in \mathbb{N}, \gamma \geq 3$. The asymptotic expansion is different for the cases: $\gamma>3$ and $\gamma=3$. The asymptotic solution contains three types of terms: the regular part, defined as in [1], the boundary layer correctors corresponding to the boundary conditions and the boundary layer correctors which realize the junction between the motion in the two rectangles. The first two types of correctors have already been introduced in $[1,2]$. The third type is characteristic for structures with junction regions. The asymptotic solution is more complicated in this case since it contains also some truncation functions introduced in order to restrict the influence of the boundary layer correctors to the regions to which they correspond. We present and solve the problems for all the components of the asymptotic solution. For the two cases $\gamma>3$ and $\gamma=3$ the order of solving the problems is presented and the leading term of the asymptotic expansion is described. In the last section, we establish the error between the exact solution and the asymptotic one, by means of the $a$ priori estimates obtained in Section 3. The small error between the two solutions justifies our asymptotic expansion.

\section{The Physical Problem}

We consider an incompressible, viscous fluid, with variable viscosity, occupying a thin domain, $\mathbb{B}^{\varepsilon}$. The flow domain is a thin tube structure, composed by two thin rectangles with lateral elastic boundaries, connected by a region with rigid boundaries. We introduce the first small parameter of our problem, $\varepsilon, \varepsilon=1 / q, q \in \mathbb{N}$ in connection with the ratio of the two dimensions of the rectangles, as below. The thin rectangles are given by:

$$
\begin{aligned}
& D_{\varepsilon}^{1}=\left\{\left(x_{1}, x_{2}\right) \in \mathbb{R}^{2}: 0<x_{1}<1,-\varepsilon<x_{2}<\varepsilon\right\}, \\
& D_{\varepsilon}^{2}=\left\{\left(x_{1}, x_{2}\right) \in \mathbb{R}^{2}:-\varepsilon<x_{1}<\varepsilon, 0<x_{2}<1\right\},
\end{aligned}
$$

and the junction region is

$$
\begin{aligned}
& D_{\varepsilon}^{r}=\left\{\left(x_{1}, x_{2}\right) \in \mathbb{R}^{2}:-\varepsilon<x_{1}<2 \varepsilon,-\varepsilon<x_{2}<2 \varepsilon\right\} \\
& \backslash\left(\left\{\left(x_{1}, x_{2}\right) \in \mathbb{R}^{2}:-\varepsilon<x_{1}<0,-\varepsilon<x_{2}<0, x_{1}^{2}+x_{2}^{2} \geq \varepsilon^{2}\right\}\right. \\
&\left.\cup\left\{\left(x_{1}, x_{2}\right) \in \mathbb{R}^{2}: \varepsilon<x_{1}<2 \varepsilon, \varepsilon<x_{2}<2 \varepsilon,\left(x_{1}-2 \varepsilon\right)^{2}+\left(x_{2}-2 \varepsilon\right)^{2} \leq \varepsilon^{2}\right\}\right) .
\end{aligned}
$$

The flow domain $\mathcal{B}^{\varepsilon} \subset \mathbb{R}^{2}$ is given by $\left(D_{\varepsilon}^{1} \cap\left\{x_{1} \geq 2 \varepsilon\right\}\right) \cup\left(D_{\varepsilon}^{2} \cap\left\{x_{2} \geq 2 \varepsilon\right\}\right) \cup D_{\varepsilon}^{r}$, as shown in Figure 3. 


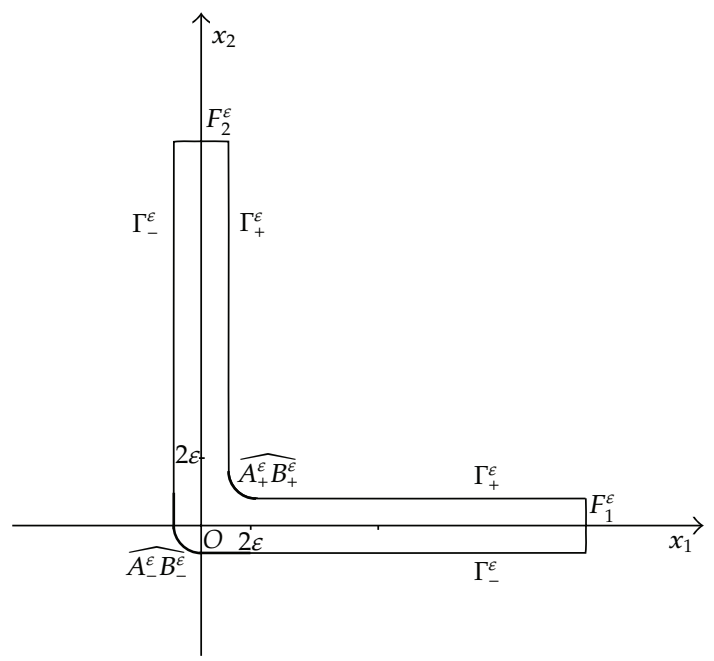

Figure 3: The domain $\mathfrak{B}^{\varepsilon}$.

The main difference between this paper and our previous works $[2,5]$ is that now the boundary of the flow domain $B^{\varepsilon}$ contains elastic parts and rigid parts as well. Let $\widehat{A_{+}^{\varepsilon} B_{+}^{\varepsilon}}$ and $A_{-}^{\varepsilon} B_{-}^{\varepsilon}$ be the rigid parts of the boundary of $B^{\varepsilon}$ defined as follows:

$$
\begin{aligned}
\widehat{A_{+}^{\varepsilon} B_{+}^{\varepsilon}=} & \left\{\left(x_{1}, x_{2}\right) \in \mathbb{R}^{2}: \varepsilon<x_{1}<2 \varepsilon, \varepsilon<x_{2}<2 \varepsilon,\left(x_{1}-2 \varepsilon\right)^{2}+\left(x_{2}-2 \varepsilon\right)^{2}=\varepsilon^{2}\right\}, \\
\widehat{A_{-}^{\varepsilon} B_{-}^{\varepsilon}=} & \left\{\left(x_{1}, x_{2}\right) \in \mathbb{R}^{2}: x_{1}=-\varepsilon, 0 \leq x_{2}<2 \varepsilon\right\} \cup\left\{\left(x_{1}, x_{2}\right) \in \mathbb{R}^{2}: 0 \leq x_{1}<2 \varepsilon, x_{2}=-\varepsilon\right\} \\
& \cup\left\{\left(x_{1}, x_{2}\right) \in \mathbb{R}^{2}:-\varepsilon<x_{1}<0,-\varepsilon<x_{2}<0, x_{1}^{2}+x_{2}^{2}=\varepsilon^{2}\right\}
\end{aligned}
$$

the elastic parts of $\partial B^{\varepsilon}$ are given by:

$$
\Gamma_{ \pm}^{\varepsilon}=\left\{\left( \pm \varepsilon, x_{2}\right): 2 \varepsilon<x_{2}<1\right\} \cup\left\{\left(x_{1}, \pm \varepsilon\right): 2 \varepsilon<x_{1}<1\right\} .
$$

Let $F_{1}^{\varepsilon}=\left\{\left(1, x_{2}\right):-\varepsilon<x_{2}<\varepsilon\right\}$ and $F_{2}^{\varepsilon}=\left\{\left(x_{1}, 1\right):-\varepsilon<x_{1}<\varepsilon\right\}$ be the inflow and outflow parts of the boundary of $\boldsymbol{B}^{\varepsilon}$. Denoting $F_{ \pm}^{\varepsilon}=\Gamma_{ \pm}^{\varepsilon} \cup A_{ \pm}^{\varepsilon} B_{ \pm}^{\varepsilon}$, we can write $\partial B^{\varepsilon}=F_{+}^{\varepsilon} \cup F_{-}^{\varepsilon} \cup F_{1}^{\varepsilon} \cup F_{2}^{\varepsilon}$.

We study the nonsteady, slow flow of the viscous fluid in the domain $B^{\varepsilon}$ previously described, when the fluid interacts with the elastic boundaries, $\Gamma_{ \pm}^{\varepsilon}$. The interaction between the fluid and the elastic boundaries produces the normal displacements $d_{ \pm}=d_{ \pm}\left(x_{1}, x_{2}, t\right)$. We neglect the tangential displacements and we consider that the elastic boundaries are clamped. We study the problem for $t \in(0, T)$, with $T$ an arbitrary positive constant independent on $\varepsilon$ and we assume that the membranes are not very elastic so that the displacement of the boundaries is small enough. Consequently, at each time $t$, we approximate the position of the elastic membranes by its initial position and, hence, the fluid flow equations are considered in 
the initial configuration. We suppose that the displacements $d_{ \pm}: F_{ \pm}^{\varepsilon} \mapsto \mathbb{R}$ have the following form:

$$
d_{ \pm}\left(x_{1}, x_{2}, t\right)= \begin{cases}d_{ \pm}\left(x_{1}, t\right) & \text { on } \bar{D}_{\varepsilon}^{1} \cap \Gamma_{ \pm}^{\varepsilon} \\ d_{ \pm}\left(x_{2}, t\right) & \text { on } \bar{D}_{\varepsilon}^{2} \cap \Gamma_{ \pm}^{\varepsilon} \\ 0 & \text { on } F_{ \pm}^{\varepsilon} \backslash \Gamma_{ \pm}^{\varepsilon}\end{cases}
$$

The problem described above, with nonhomogeneous boundary conditions for the velocity, is modeled by the following coupled system:

$$
\begin{aligned}
& \rho_{f} \frac{\partial \mathbf{u}}{\partial t}-2 \operatorname{div}_{x}\left(\mathcal{v}(x) D_{x} \mathbf{u}\right)+\nabla_{x} p=\mathbf{f} \quad \text { in } 乃^{\varepsilon} \times(0, T), \\
& \operatorname{div}_{x} \mathbf{u}=0 \text { in } 乃^{\varepsilon} \times(0, T), \\
& \rho h \frac{\partial^{2} d_{ \pm}}{\partial t^{2}}+\frac{h^{3} E}{12} \frac{\partial^{4} d_{ \pm}}{\partial x_{i}^{4}}+\mu \frac{\partial^{5} d_{ \pm}}{\partial x_{i}^{4} \partial t}=g_{ \pm} \pm p_{\left.\right|_{\bar{D}_{\varepsilon}^{i} \cap \Gamma_{ \pm}^{\varepsilon}} ^{\varepsilon}} \quad \text { on }\left(\bar{D}_{\varepsilon}^{i} \cap \Gamma_{ \pm}^{\varepsilon}\right) \times(0, T), i=1,2, \\
& d_{+}=0 \quad \text { on } A_{+}^{\widehat{\varepsilon}} B_{+}^{\varepsilon} \times(0, T), \\
& d_{-}=0 \text { on } \widehat{A_{-}^{\varepsilon}} B_{-}^{\varepsilon} \times(0, T), \\
& \mathbf{u}=\boldsymbol{\psi}_{\varepsilon} \quad \text { on }\left(\partial \boldsymbol{B}^{\varepsilon} \backslash\left(\Gamma_{+}^{\varepsilon} \cup \Gamma_{-}^{\varepsilon}\right)\right) \times(0, T), \\
& d_{ \pm}(1, \pm \varepsilon, t)=\frac{\partial d_{ \pm}}{\partial x_{1}}(1, \pm \varepsilon, t)=0 \quad \text { in }(0, T) \\
& d_{ \pm}(2 \varepsilon, \pm \varepsilon, t)=\frac{\partial d_{ \pm}}{\partial x_{1}}(2 \varepsilon, \pm \varepsilon, t)=0 \quad \text { in }(0, T), \\
& d_{ \pm}( \pm \varepsilon, 1, t)=\frac{\partial d_{ \pm}}{\partial x_{2}}( \pm \varepsilon, 1, t)=0 \quad \text { in }(0, T) \\
& d_{ \pm}( \pm \varepsilon, 2 \varepsilon, t)=\frac{\partial d_{ \pm}}{\partial x_{2}}( \pm \varepsilon, 2 \varepsilon, t)=0 \quad \text { in }(0, T), \\
& \mathbf{u} \cdot \mathbf{n}= \pm \frac{\partial d_{ \pm}}{\partial t}, \quad \mathbf{u} \cdot \boldsymbol{\tau}=0 \quad \text { on } \Gamma_{ \pm}^{\varepsilon} \times(0, T), \\
& \mathbf{u}(x, 0)=\mathbf{0} \text { in } \mathbb{B}^{\varepsilon}, \\
& d_{ \pm}(x, 0)=\frac{\partial d_{ \pm}}{\partial t}(x, 0)=0 \quad \text { on } \Gamma_{ \pm}^{\varepsilon},
\end{aligned}
$$

with $\mathbf{n}$ the outer unit normal on the boundary of $\boldsymbol{B}^{\varepsilon}$ and $\boldsymbol{\tau}$ the unit tangent vector to $\partial \mathbb{B}^{\varepsilon}$. The given data contained by the previous system are: some material constants and some given functions. $\rho_{f}, \rho, \mu, E$ are positive given constants in connection with the properties of materials representing the density of the fluid, the density of the elastic walls, a viscosity coefficient, and the two-dimensional Young's modulus (The two dimensional Young's modulus 
$E$ is defined as $E^{(3)} /\left(1-\widehat{v}^{2}\right)$ where $E^{(3)}$ is the common three dimensional Young modulus and $\widehat{v}$ is the Poisson ratio.), respectively, and the positive constant $h$ stands for the thickness of the elastic walls.

The given functions are: $v$, the variable viscosity of the fluid, which satisfies $v \in C^{1}\left(\mathbb{B}^{\varepsilon}\right)$, $v(x) \geq \alpha>0$ for all $x \in \mathbb{B}^{\varepsilon}, \mathbf{f}$, the exterior force applied to the fluid, $g_{ \pm}$, the exterior forces applied on the elastic walls, with $g_{ \pm}: F_{ \pm}^{\varepsilon} \mapsto \mathbb{R}$,

$$
g_{ \pm}\left(x_{1}, x_{2}, t\right)=\left\{\begin{array}{lc}
g_{ \pm}\left(x_{1}, t\right) & \text { on } \bar{D}_{\varepsilon}^{1} \cap \Gamma_{ \pm \prime}^{\varepsilon} \\
g_{ \pm}\left(x_{2}, t\right) & \text { on } \bar{D}_{\varepsilon}^{2} \cap \Gamma_{ \pm \prime}^{\varepsilon} \\
0 & \text { on } F_{ \pm}^{\varepsilon} \backslash \Gamma_{ \pm}^{\varepsilon}
\end{array}\right.
$$

and a small inflow-outflow velocity defined by means of the function $\psi_{\varepsilon}$ defined as follows:

$$
\boldsymbol{\Psi}_{\varepsilon}\left(x_{1}, x_{2}, t\right)=\varepsilon^{2} \boldsymbol{\Psi}\left(\xi_{1}, \xi_{2}, t\right), \quad \forall x \in \mathbb{B}^{\varepsilon},
$$

with $\left(\xi_{1}, \xi_{2}\right)=\left(x_{1} / \varepsilon, x_{2} / \varepsilon\right)$. The function $\boldsymbol{\psi}$ is the trace of a function denoted also by $\boldsymbol{\psi}$ with the following properties:

$$
\begin{array}{r}
\boldsymbol{\psi}\left(\xi_{1}, \xi_{2}, t\right)=\psi\left(\xi_{2}, t\right) \mathbf{e}_{1}, \quad \forall\left(\xi_{1}, \xi_{2}\right) \in(2, \infty) \times(-1,1), \\
\boldsymbol{\psi}\left(\xi_{1}, \xi_{2}, t\right)=\psi\left(\xi_{1}, t\right) \mathbf{e}_{2}, \quad \forall\left(\xi_{1}, \xi_{2}\right) \in(-1,1) \times(2, \infty), \\
\operatorname{div}_{\xi} \boldsymbol{\psi}=0, \quad \text { in } D^{r}, \\
\boldsymbol{\psi}=\mathbf{0} \quad \text { on } F_{+} \cup F_{-}, \\
\boldsymbol{\psi}\left(\xi_{1}, \xi_{2}, 0\right)=\mathbf{0}, \\
\int_{-1}^{1} \psi\left(\xi_{2}, t\right) \mathrm{d} \xi_{2}+\int_{-1}^{1} \psi\left(\xi_{1}, t\right) \mathrm{d} \xi_{1}=0
\end{array}
$$

where $D^{r}=(1 / \varepsilon) D_{\varepsilon}^{r}, F_{ \pm}=F_{ \pm}^{\varepsilon}$. The unknowns of the system (2.6) are the velocity of the fluid, $\mathbf{u}$, the pressure of the fluid, $p$, and the normal displacements of the elastic walls, $d_{ \pm}$.

The fluid flow is described by the nonsteady Stokes equations. For the normal displacements we consider the Sophie-Germain's equation. A "viscous" type term, $\mu\left(\partial^{5} d_{ \pm}^{i} / \partial x_{i}^{4} \partial t\right)$, is added to the usual forth-order equation for the normal displacements. It corresponds to the viscoelastic behavior of the wall (the so called Kelvin-Voigt model). Usually, the Young's modulus, $E$, has a value of $10^{4}-10^{6} \mathrm{~Pa}$. On the other hand, we assume that the characteristic longitudinal space scale for vessels is of order of $\mathrm{cm}$ and the time scale is of order of seconds. Let us use the S.I. system of units. This leads us to the necessity of scaling of every derivative is $x_{i}$ by the factor $10^{2}$; that is, the fourth derivative will contain the additional factor $10^{8}$. If $h$ is of order $10^{-3} \mathrm{~m}$ or $10^{-2} \mathrm{~m}$, then the coefficient $\rho h$ can be taken in the further analysis as a value of order of 1 . The coefficient $h^{3} E / 12$ in $(2.6)_{3}$ will be replaced (after scaling in $x_{i}$ ) by a great coefficient $\delta^{-1}$ with the value $\delta$ of order of $10^{-7}$ to $10^{-4}$. If the ratio of thickness and the length of the vessel $\varepsilon$ are of order $10^{-2}$, then $\delta$ is of order from $\varepsilon^{2}$ to $\varepsilon^{4}$. We assume that the "viscous" term is much smaller than the term with the coefficient $\delta^{-1}$ and hence the new 
coefficient denoted also by $\mu$, obtained after scaling in $x_{i}$, is $O(1)$. More details concerning (2.6) can be found, for instance in [1].

Due to the properties of the function $\boldsymbol{\psi}_{\varepsilon_{\varepsilon}}$, the compatibility condition for the coupled system which describes the physical problem is

$$
0=\int_{\partial \mathbb{B}^{\varepsilon}} \mathbf{u}(x, t) \cdot \mathbf{n d} s=\frac{\mathrm{d}}{\mathrm{d} t}\left(\int_{2 \varepsilon}^{1}\left(d_{+}\left(x_{1}, t\right)-d_{-}\left(x_{1}, t\right)\right) \mathrm{d} x_{1}+\int_{2 \varepsilon}^{1}\left(d_{+}\left(x_{2}, t\right)-d_{-}\left(x_{2}, t\right)\right) \mathrm{d} x_{2}\right) .
$$

Using next the initial condition for the displacements, condition for the above coupled system becomes

$$
\int_{2 \varepsilon}^{1}\left(d_{+}\left(x_{1}, t\right)-d_{-}\left(x_{1}, t\right)\right) \mathrm{d} x_{1}+\int_{2 \varepsilon}^{1}\left(d_{+}\left(x_{2}, t\right)-d_{-}\left(x_{2}, t\right)\right) \mathrm{d} x_{2}=0
$$

This condition states that the global area of the flow domain is preserved.

\section{Variational Formulation: Existence, Uniqueness, Regularity, and A Priori Estimates}

In order to obtain the above properties for the solution of the physical problem, we introduce the variational framework.

To simplify the computations, we consider first (2.6) with homogeneous boundary conditions on $F_{1}^{\varepsilon}$ and $F_{2}^{\varepsilon}$, that is, the problem for $\boldsymbol{\psi}_{\varepsilon}=0$. Then, the same properties for the solution of (2.6) follow with the usual technique for nonhomogeneous problems.

Taking into account the conditions for the velocity and for the displacements and the condition (2.11) we introduce the following spaces:

$$
\begin{aligned}
V^{\varepsilon}= & \left\{\mathbf{v} \in\left(H^{1}\left(\mathcal{B}^{\varepsilon}\right)\right)^{2}: \operatorname{div} \mathbf{v}=0 \text { in }{B^{\varepsilon}}^{\varepsilon}, \mathbf{v}=\mathbf{0} \text { on } \partial B^{\varepsilon} \backslash\left(\Gamma_{+}^{\varepsilon} \cup \Gamma_{-}^{\varepsilon}\right), \mathbf{v} \cdot \boldsymbol{\tau}=0 \text { on } \Gamma_{ \pm}^{\varepsilon}\right\}, \\
W^{\varepsilon}= & \left\{\left(\beta_{+}, \beta_{-}\right) \in H^{1}\left(F_{+}^{\varepsilon}\right) \times H^{1}\left(F_{-}^{\varepsilon}\right):\left(\beta_{+}, \beta_{-}\right) \in H_{0}^{2}\left(\Gamma_{+}^{\varepsilon}\right) \times H_{0}^{2}\left(\Gamma_{-}^{\varepsilon}\right),\left(\beta_{+}, \beta_{-}\right)=(0,0)\right. \\
& \text { on } \left.\widehat{A_{+}^{\varepsilon}} B_{+}^{\varepsilon} \times A_{-}^{\varepsilon} B_{-}^{\varepsilon}, \int_{2 \varepsilon}^{1}\left(\beta_{+}^{1}\left(x_{1}\right)-\beta_{-}^{1}\left(x_{1}\right)\right) \mathrm{d} x_{1}+\int_{2 \varepsilon}^{1}\left(\beta_{+}^{2}\left(x_{2}\right)-\beta_{-}^{2}\left(x_{2}\right)\right) \mathrm{d} x_{2}=0\right\} .
\end{aligned}
$$


Choosing for the data the regularity $\mathbf{f} \in L^{2}\left(0, T ;\left(L^{2}\left(\mathcal{B}^{\varepsilon}\right)\right)^{2}\right)$ and $\mathbf{g}=\left(g_{+}, g_{-}\right) \in L^{2}\left(0, T ; F_{+}^{\varepsilon}\right) \times$ $L^{2}\left(0, T ; F_{-}^{\varepsilon}\right)$ we consider the following variational problem:

Find $(\mathbf{u}, \mathbf{d}) \in L^{2}\left(0, T ; V^{\varepsilon}\right) \times H^{1}\left(0, T ; W^{\varepsilon}\right)$, with $(\dot{\mathbf{u}}, \ddot{\mathbf{d}}) \in L^{2}\left(0, T ;\left(V^{\varepsilon}\right)^{\prime}\right) \times L^{2}\left(0, T ;\left(W^{\varepsilon}\right)^{\prime}\right)$

which satisfies a.e. in $(0, T)$ :

$$
\begin{aligned}
& \rho_{f} \frac{\mathrm{d}}{\mathrm{d} t} \int_{B^{\varepsilon}} \mathbf{u} \cdot \boldsymbol{\varphi} \mathrm{d} x+2 \int_{B^{\varepsilon}} v \mathrm{Du}: \mathrm{D} \boldsymbol{\varphi} \mathrm{d} x \\
& \quad+\sum_{i=1}^{2}\left\{\rho h \frac{\mathrm{d}}{\mathrm{d} t} \int_{2 \varepsilon}^{1} \frac{\partial \mathrm{d}}{\partial t} \cdot \boldsymbol{\beta} \mathrm{d} x_{i}+\frac{h^{3} E}{12} \int_{2 \varepsilon}^{1} \frac{\partial^{2} \mathbf{d}}{\partial x_{i}^{2}} \cdot \frac{\partial^{2} \boldsymbol{\beta}}{\partial x_{i}^{2}} \mathrm{~d} x_{i}+\mu \int_{2 \varepsilon}^{1} \frac{\partial^{3} \mathrm{~d}}{\partial x_{i}^{2} \partial t} \cdot \frac{\partial^{2} \boldsymbol{\beta}}{\partial x_{i}^{2}} \mathrm{~d} x_{i}\right\} \\
& \quad=\int_{B^{\varepsilon}} \mathbf{f} \cdot \boldsymbol{\varphi} \mathrm{d} x+\sum_{i=1}^{2} \int_{2 \varepsilon}^{1} \mathbf{g} \cdot \boldsymbol{\beta} \mathrm{d} x_{i} \\
& \forall \boldsymbol{\varphi} \in V^{\varepsilon}, \forall \boldsymbol{\beta} \in W^{\varepsilon}, \text { with } \boldsymbol{\varphi} \cdot \mathbf{n}= \pm \beta_{ \pm} \text {on } \Gamma_{ \pm \prime}^{\varepsilon} \\
& \mathbf{u} \cdot \mathbf{n}= \pm \frac{\partial d_{ \pm}}{\partial t} \quad \text { on } \Gamma_{ \pm \prime}^{\varepsilon} \\
& \mathbf{u}(0)=\mathbf{0}, \quad \mathbf{d}(0)=\dot{\mathbf{d}}(0)=\mathbf{0} .
\end{aligned}
$$

Here and below $\mathbf{d}=\left(d_{+}, d_{-}\right)$and $\boldsymbol{\beta}=\left(\beta_{+}, \beta_{-}\right)$.

For the nonhomogeneous boundary conditions we still obtain the variational formulation (3.2) with $\mathbf{u}$ replaced by $\mathbf{u}-\boldsymbol{\psi}_{\varepsilon}$ and $\mathbf{f}$ replaced by $\mathbf{f}-\rho_{f}\left(\partial \boldsymbol{\psi}_{\varepsilon} / \partial t\right)+2 \operatorname{div}\left(v D \boldsymbol{\psi}_{\varepsilon}\right)$.

Theorem 3.1. The variational problem (3.2) has the unique solution $(\mathbf{u}, \mathbf{d})$ with $(\dot{\mathbf{u}}, \ddot{\mathbf{d}}) \in L^{2}(0, T$; $\left.\left(L^{2}\left(B^{\varepsilon}\right)\right)^{2}\right) \times L^{2}\left(0, T ; L^{2}\left(F_{+}^{\varepsilon}\right) \times L^{2}\left(F_{-}^{\varepsilon}\right)\right)$.

Proof. Let us start with the proof of the uniqueness for the solution of (3.2). Consider $\left(\mathbf{u}_{1}, \mathbf{d}_{1}\right)$ and $\left(\mathbf{u}_{2}, \mathbf{d}_{2}\right)$ two solutions of (3.2) and define $(\mathbf{u}, \mathbf{d})=\left(\mathbf{u}_{1}-\mathbf{u}_{2}, \mathbf{d}_{1}-\mathbf{d}_{2}\right)$. Subtracting the two relations $(3.2)_{1}$ and taking as test function $(\boldsymbol{\varphi}, \boldsymbol{\beta})=(\mathbf{u}, \mathbf{d})$ we get:

$$
\begin{aligned}
& \frac{\rho_{f}}{2} \frac{\mathrm{d}}{\mathrm{d} t} \int_{\mathbb{B}^{\varepsilon}} \mathbf{u}^{2} \mathrm{~d} x+2 \int_{B^{\varepsilon}} v \text { Du }: \text { D } \mathbf{u} \mathrm{d} x \\
& \quad+\sum_{i=1}^{2}\left(\frac{\rho h}{2} \frac{\mathrm{d}}{\mathrm{d} t} \int_{2 \varepsilon}^{1}\left(\frac{\partial \mathrm{d}}{\partial t}\right)^{2} \mathrm{~d} x_{i}+\frac{h^{3} E}{24} \frac{\mathrm{d}}{\mathrm{d} t} \int_{2 \varepsilon}^{1}\left(\frac{\partial^{2} \mathbf{d}}{\partial x_{i}^{2}}\right)^{2} \mathrm{~d} x_{i}+\mu \int_{2 \varepsilon}^{1}\left(\frac{\partial^{3} \mathrm{~d}}{\partial x_{i}^{2} \partial t}\right)^{2} \mathrm{~d} x_{i}\right)=0 .
\end{aligned}
$$

Integrating from 0 to $t$ this equality and taking into account the initial conditions, we obtain: $\mathbf{u}=\mathbf{0}$ a.e. in $(0, T)$ and $\mathbf{d}=\mathbf{0}$ a.e. in $(0, T)$. Hence the problem (3.2) has a unique solution.

For proving the existence and the regularity of the functions $\mathbf{u}$ and $\mathbf{d}$, we will use the Galerkin's method. 
We begin with the construction of a basis for the space $W^{\varepsilon}$. Let $\left\{\zeta_{j}\right\}_{j \in \mathbb{N}}$ be a basis of $H_{0}^{2}(2 \varepsilon, 1)$ chosen by considering the eigenfunctions of the following problem:

$$
\begin{gathered}
\zeta_{j}^{(i v)}=\alpha_{j} \zeta_{j} \quad \text { in }(2 \varepsilon, 1), \\
\zeta_{j}(2 \varepsilon)=\zeta_{j}(1)=0 \\
\zeta_{j}^{\prime}(2 \varepsilon)=\zeta_{j}^{\prime}(1)=0
\end{gathered}
$$

where $\zeta_{j}^{(i v)}$ is the fourth derivative of $\zeta_{j}$ and $\alpha_{j}>0$, for all $j \in \mathbb{N}$. We define $\left\{\boldsymbol{\beta}_{j}\right\}_{j \in \mathbb{N}}$ as follows: $\boldsymbol{\beta}_{j}=\left(\beta_{+_{j}}, \beta_{-_{j}}\right)$ where

$$
\beta_{ \pm j}\left(x_{1}, x_{2}\right)= \begin{cases}\zeta_{j}\left(x_{1}\right) & \text { for }\left(x_{1}, x_{2}\right) \in(2 \varepsilon, 1) \times\{ \pm \varepsilon\} \\ 0 & \text { for }\left(x_{1}, x_{2}\right) \in \widehat{A_{ \pm}^{\varepsilon} B_{ \pm \prime}^{\varepsilon}} \\ \zeta_{j}\left(x_{2}\right) & \text { for }\left(x_{1}, x_{2}\right) \in\{ \pm \varepsilon\} \times(2 \varepsilon, 1)\end{cases}
$$

It is easy to check that $\left\{\boldsymbol{\beta}_{j}\right\}_{j \in \mathbb{N}}$ is a basis for $W^{\varepsilon}$. We choose the functions of the basis $\left\{\zeta_{j}\right\}_{j \in \mathbb{N}}$ such that

$$
\int_{B^{\varepsilon}} \boldsymbol{\beta}_{i} \cdot \boldsymbol{\beta}_{k} \mathrm{~d} x=\delta_{i k}
$$

As a consequence of the previous relation we also get:

$$
\int_{B^{\varepsilon}} \boldsymbol{\beta}_{i}^{\prime \prime} \cdot \boldsymbol{\beta}_{k}^{\prime \prime}=\alpha_{k} \delta_{i k}
$$

We consider now $\left\{\boldsymbol{\Psi}_{i}\right\}_{i \in \mathbb{N}}$ a basis of the space $V_{0}^{\varepsilon}=\left\{\mathbf{u} \in\left(H_{0}^{1}\left(\mathbb{B}^{\varepsilon}\right)\right)^{2}: \operatorname{div} \mathbf{u}=0\right.$ in $\left.\mathcal{B}^{\varepsilon}\right\}$, constructed with the eigenfunctions of the following Stokes problem:

$$
\begin{gathered}
-2 \operatorname{div}\left(v \mathrm{D} \boldsymbol{\Psi}_{i}\right)+\nabla q_{i}=\lambda_{i} \boldsymbol{\Psi}_{i} \quad \text { in } \boldsymbol{B}^{\varepsilon}, \\
\operatorname{div} \boldsymbol{\Psi}_{i}=0 \quad \text { in } \boldsymbol{B}^{\varepsilon}, \\
\boldsymbol{\Psi}_{i}=0 \quad \text { on } \partial \boldsymbol{B}^{\varepsilon},
\end{gathered}
$$

with $\lambda_{i}>0$ for all $i \in \mathbb{N}$. The functions $\boldsymbol{\psi}_{i}, i \in \mathbb{N}$ are uniquely determined from the condition

$$
\int_{B^{\varepsilon}} \boldsymbol{\psi}_{j} \cdot \boldsymbol{\psi}_{k} \mathrm{~d} x=\delta_{j k}
$$

which implies

$$
2 \int_{B^{\varepsilon}} v \mathrm{D} \boldsymbol{\psi}_{i}: \mathrm{D} \boldsymbol{\psi}_{k} \mathrm{~d} x=\lambda_{k} \delta_{j k}
$$


Next, for any $\boldsymbol{\beta}_{j}$ we consider the following problem:

Find $\left(\boldsymbol{\varphi}_{j}, p_{j}\right) \in V^{\varepsilon} \times L^{2}\left(\mathcal{B}^{\varepsilon}\right)$ such that

$$
\begin{aligned}
& -2 \operatorname{div}\left(v \mathrm{D}\left(\boldsymbol{\varphi}_{j}\right)\right)+\nabla p_{j}=0 \quad \text { in }\left(H^{-1}\left(B^{\varepsilon}\right)\right)^{2}, \\
& \boldsymbol{\varphi}_{j} \cdot \mathbf{n}= \pm \beta_{ \pm j} \text { on } \Gamma_{ \pm}^{\varepsilon} .
\end{aligned}
$$

Following the classical results of [13] for nonhomogeneous Stokes problems, we obtain a unique $\boldsymbol{\varphi}_{j}$ and a function $p_{j}$ unique up to an additive constant. Moreover, for any $j, k \in \mathbb{N}$

$$
\int_{B^{\varepsilon}} v \mathrm{D} \boldsymbol{\varphi}_{j}: \mathrm{D} \boldsymbol{\varphi}_{k} \mathrm{~d} x=0
$$

By means of the functions $\left\{\boldsymbol{\beta}_{j}\right\}_{j \in \mathbb{N}},\left\{\boldsymbol{\varphi}_{i}\right\}_{i \in \mathbb{N}},\left\{\boldsymbol{\varphi}_{j}\right\}_{j \in \mathbb{N}}$, we are now in a position to define, for each $n, m \in \mathbb{N}$, an approximate solution $\left(\mathbf{u}_{n}^{m}, \mathbf{d}_{n}\right)$ of (3.2) as follows:

$$
\begin{gathered}
\mathbf{u}_{n}^{m}(x, t)=\sum_{i=1}^{m} a_{i}(t) \boldsymbol{\varphi}_{i}(x)+\sum_{j=1}^{n} \dot{b}_{j}(t) \boldsymbol{\varphi}_{j}(x), \\
\mathbf{d}_{n}(x, t)=\sum_{j=1}^{n} b_{j}(t) \boldsymbol{\beta}_{j}(x),
\end{gathered}
$$

with $a_{i}, b_{j}:[0, T] \mapsto \mathbb{R}, i=1, \ldots, m, j=1, \ldots, n$ scalar unknown functions. These functions are determined below from the condition that $\left(\mathbf{u}_{n}^{m}, \mathbf{d}_{n}\right)$ is the solution for the problem:

$$
\begin{aligned}
& \rho_{f} \int_{B^{\varepsilon}} \frac{\partial \mathbf{u}_{n}^{m}}{\partial t} \cdot \boldsymbol{\psi}_{i} \mathrm{~d} x+2 \int_{B^{\varepsilon}} v D \mathbf{u}_{n}^{m}: \mathrm{D} \boldsymbol{\psi}_{i} \mathrm{~d} x=\int_{B^{\varepsilon}} \mathbf{f} \cdot \boldsymbol{\psi}_{i} \mathrm{~d} x, \quad \text { for } i \in\{1, \ldots, m\}, \\
& \rho_{f} \int_{B^{\varepsilon}} \frac{\partial \mathbf{u}_{n}^{m}}{\partial t} \cdot \boldsymbol{\varphi}_{j} \mathrm{~d} x+2 \int_{B^{\varepsilon}} v \mathrm{D} \mathbf{u}_{n}^{m}: \mathrm{D} \boldsymbol{\varphi}_{j} \mathrm{~d} x \\
& +\sum_{l=1}^{2}\left(\rho h \int_{2 \varepsilon}^{1} \frac{\partial^{2} \mathbf{d}_{n}}{\partial t^{2}} \cdot \boldsymbol{\beta}_{j} \mathrm{~d} x_{l}+\frac{h^{3} E}{12} \int_{2 \varepsilon}^{1} \frac{\partial^{2} \mathbf{d}_{n}}{\partial x_{l}^{2}} \cdot \frac{\partial^{2} \boldsymbol{\beta}_{j}}{\partial x_{l}^{2}} \mathrm{~d} x_{l}+\mu \int_{2 \varepsilon}^{1} \frac{\partial^{3} \mathbf{d}_{n}}{\partial x_{l}^{2} \partial t} \cdot \frac{\partial^{2} \boldsymbol{\beta}_{\mathbf{j}}}{\partial x_{l}^{2}} \mathrm{~d} x_{l}\right) \\
& =\int_{B^{\varepsilon}} \mathbf{f} \cdot \boldsymbol{\varphi}_{j} \mathrm{~d} x+\sum_{l=1}^{2} \int_{2 \varepsilon}^{1} \mathbf{g} \cdot \boldsymbol{\beta}_{j} \mathrm{~d} x_{l}, \quad \text { for } j \in\{1, \ldots, n\}, \\
& \mathbf{u}_{n}^{m} \cdot \mathbf{n}= \pm \frac{\partial d_{n}^{ \pm}}{\partial t} \quad \text { on } \Gamma_{ \pm}^{\varepsilon} \\
& \mathbf{u}_{n}^{m}(0)=\mathbf{0}, \quad \mathbf{d}_{n}(0)=\dot{\mathbf{d}}_{n}(0)=\mathbf{0} .
\end{aligned}
$$


We introduce the notations:

$$
p_{i k}=\int_{B^{\varepsilon}} \boldsymbol{\varphi}_{k} \cdot \boldsymbol{\varphi}_{i} \mathrm{~d} x, \quad q_{k j}=\int_{B^{\varepsilon}} \boldsymbol{\varphi}_{k} \cdot \boldsymbol{\varphi}_{j} \mathrm{~d} x, \quad r_{i k}=2 \int_{B^{\varepsilon}} v \mathrm{D} \boldsymbol{\varphi}_{i}: \mathrm{D} \boldsymbol{\varphi}_{k} \mathrm{~d} x .
$$

Taking into account the previous notations and the relations (3.6), (3.7), (3.9), (3.10), (3.12) we get from (3.14) the following $m+n$ linear differential system for the unknown functions $a_{i}, b_{j}, i=1, \ldots, m, j=1, \ldots, n$ :

$$
\begin{aligned}
& \rho_{f} \dot{a}_{i}(t)+\lambda_{i} a_{i}(t)+\sum_{k=1}^{n} p_{i k} \ddot{b}_{k}(t)=\int_{\mathcal{B}^{\varepsilon}} \mathbf{f} \cdot \boldsymbol{\Psi}_{i} \mathrm{~d} x, \\
& \rho_{f} \sum_{k=1}^{m} p_{k j} \dot{a}_{k}(t)+\rho_{f} \sum_{k=1}^{m} q_{k j} \ddot{b}_{k}(t)+\sum_{k=1}^{n} r_{k j} \dot{b}_{k}(t)+\rho h \ddot{b}_{j}(t)+\frac{h^{3} E}{12} \alpha_{j} b_{j}(t)+\mu \alpha_{j} \dot{b}_{j}(t) \\
& \quad=\int_{B^{\varepsilon}} \mathbf{f} \cdot \boldsymbol{\varphi}_{j} \mathrm{~d} x+\sum_{l=1}^{2} \int_{2 \varepsilon}^{1} \mathbf{g} \cdot \boldsymbol{\beta}_{j} \mathrm{~d} x_{l}, \\
& a_{i}(0)=b_{j}(0)=\dot{b}_{j}(0)=0, \quad i=1, \ldots, m, j=1, \ldots, n .
\end{aligned}
$$

The previous system uniquely determines the unknown functions $a_{i}, b_{j}, i=1, \ldots, m, j=$ $1, \ldots, n$. For more details see $[1$, Section 3$]$.

In the sequel we obtain some a priori estimates which give the regularity of the solution for (3.2). Computing $\sum_{i=1}^{m} a_{i}(t) \cdot(3.14)_{1}+\sum_{j=1}^{n} \dot{b}_{j}(t) \cdot(3.14)_{2}$ and using (3.13) we get:

$$
\begin{aligned}
& \frac{\rho_{f}}{2} \frac{\mathrm{d}}{\mathrm{d} t} \int_{B^{\varepsilon}}\left(\mathbf{u}_{n}^{m}\right)^{2} \mathrm{~d} x+\int_{B^{\varepsilon}} v\left(D \mathbf{u}_{n}^{m}\right)^{2} \\
& \quad+\sum_{i=1}^{2}\left(\frac{\rho h}{2} \frac{\mathrm{d}}{\mathrm{d} t} \int_{2 \varepsilon}^{1}\left(\frac{\partial \mathbf{d}_{n}}{\partial t}\right)^{2} \mathrm{~d} x_{i}+\frac{h^{3} E}{24} \frac{\mathrm{d}}{\mathrm{d} t} \int_{2 \varepsilon}^{1}\left(\frac{\partial^{2} \mathbf{d}_{n}}{\partial x_{i}^{2}}\right)^{2} \mathrm{~d} x_{i}+\mu \int_{2 \varepsilon}^{1}\left(\frac{\partial^{3} \mathbf{d}_{n}}{\partial x_{i}^{2} \partial t}\right)^{2} \mathrm{~d} x_{i}\right) \\
& \quad=\int_{B^{\varepsilon}} \mathbf{f} \cdot \mathbf{u}_{n}^{m} \mathrm{~d} x+\sum_{i=1}^{2} \int_{2 \varepsilon}^{1} \mathbf{g} \cdot \frac{\partial \mathbf{d}_{n}}{\partial t} \mathrm{~d} x_{i} .
\end{aligned}
$$


Integrating from 0 to $t$, using the property of $v$ and the initial conditions we obtain, as in [1], the first estimates:

$$
\begin{aligned}
&\left\|\mathbf{u}_{m}^{n}\right\|_{L^{\infty}\left(0, T ;\left(L^{2}\left(\mathcal{B}^{\varepsilon}\right)\right)^{2}\right)} \leq C(\mathbf{f}, \mathbf{g}), \\
&\left\|\mathrm{D} \mathbf{u}_{m}^{n}\right\|_{L^{2}\left(0, T ;\left(L^{2}\left(\mathcal{B}^{\varepsilon}\right)\right)^{4}\right)} \leq C(\mathbf{f}, \mathbf{g}), \\
&\left\|\frac{\partial d_{ \pm n}}{\partial t}\right\|_{L^{\infty}\left(0, T ; L^{2}\left(\Gamma_{ \pm}^{\varepsilon}\right)\right)} \leq C(\mathbf{f}, \mathbf{g}), \\
&\left\|\frac{\partial^{2} d_{ \pm n}}{\partial s^{2}}\right\|_{L^{\infty}\left(0, T ; L^{2}\left(\Gamma_{ \pm}^{\varepsilon}\right)\right)} \leq C(\mathbf{f}, \mathbf{g}), \\
&\left\|\frac{\partial^{3} d_{ \pm n}}{\partial s^{2} \partial t}\right\|_{L^{2}\left(\Gamma_{ \pm}^{\varepsilon} \times(0, T)\right)} \leq C(\mathbf{f}, \mathbf{g}),
\end{aligned}
$$

with $C(\mathbf{f}, \mathbf{g})=C\left(T, \mu, \rho_{f}, \rho, E, h\right)\left(\|\mathbf{f}\|_{L^{2}\left(0, T ;\left(L^{2}\left(\mathcal{B}^{e}\right)\right)^{2}\right)}+\|\mathbf{g}\|_{L^{2}\left((0, T) ; L^{2}\left(F_{+}^{\varepsilon} \times F_{-}^{\varepsilon}\right)\right)}\right)$ and $s$ is the variable of the parametrisation for $\Gamma_{+}^{\varepsilon}$ or on $\Gamma_{-}^{\varepsilon}$.

The second estimates are obtained computing $\sum_{i=1}^{m} \dot{a}_{i}(t) \cdot(3.14)_{1}+\sum_{j=1}^{n} \ddot{b}_{j}(t) \cdot(3.14)_{2}$ :

$$
\begin{aligned}
\left\|\frac{\partial \mathbf{u}_{m}^{n}}{\partial t}\right\|_{L^{2}\left(0, T ;\left(L^{2}\left(\mathcal{B}^{\varepsilon}\right)\right)^{2}\right)} & \leq\left(\frac{h^{3} E}{12}\right)^{1 / 2} C(\mathbf{f}, \mathbf{g}) \\
\left\|\mathrm{D} \mathbf{u}_{m}^{n}\right\|_{L^{\infty}\left((0, T) ;\left(L^{2}\left(\mathcal{B}^{\varepsilon}\right)\right)^{4}\right)} & \leq\left(\frac{h^{3} E}{12}\right)^{1 / 2} C(\mathbf{f}, \mathbf{g}) \\
\left\|\frac{\partial^{2} d_{ \pm n}}{\partial t^{2}}\right\|_{L^{2}\left(\Gamma_{ \pm}^{e} \times(0, T)\right)} \leq\left(\frac{h^{3} E}{12}\right)^{1 / 2} C(\mathbf{f}, \mathbf{g}) & \leq\left(\frac{h^{3} E}{12}\right)^{1 / 2} C(\mathbf{f}, \mathbf{g}) .
\end{aligned}
$$

From (3.18) $)_{1,2}$ and (3.19) $)_{1,2}$ we get the boundedness of $\left\{\mathbf{u}_{n}^{m}\right\}_{m, n \in \mathbb{N}}$ in $L^{\infty}\left(0, T ; V^{\varepsilon}\right) \cap H^{1}(0, T$; $\left.\left(L^{2}\left(B^{\varepsilon}\right)\right)^{2}\right)$. Consequently, we can pass to the limit in (3.14) which yields the existence result of the theorem. To achieve the proof, we notice that the regularity stated in (3.2) follows from the estimates (3.18) and (3.19).

As a consequence of Theorem 3.1 we introduce the pressure which appears in $(2.6)_{1,3}$.

Corollary 3.2. There exists a unique function $p \in L^{2}\left(0, T ; H^{1}\left(\mathbb{B}^{\varepsilon}\right)\right)$ such that $(\mathbf{u}, p, \mathbf{d})$ satisfies $(2.6)_{1,3}$ a.e. in $B^{\varepsilon} \times(0, T)$ and on $\left(\bar{D}_{\varepsilon}^{i} \cap \Gamma_{ \pm}^{\varepsilon}\right) \times(0, T)$, respectively.

To obtain this result we follow the ideas of [1, Section 3].

The last result of this section presents the estimates for $(\mathbf{u}, p, \mathbf{d})$, the unique solution of (2.6). 
Corollary 3.3. Let $(\mathbf{u}, p, \mathbf{d})$ be the solution of the problem (2.6) corresponding to the data $\mathbf{f}, \mathbf{g}$, with $\mathbf{f} \in L^{2}\left(0, T ;\left(L^{2}\left(\mathbb{B}^{\varepsilon}\right)\right)^{2}\right)$ and $\mathbf{g} \in L^{2}\left(0, T ; F_{+}^{\varepsilon}\right) \times L^{2}\left(0, T ; F_{-}^{\varepsilon}\right)$. Then the following estimates hold:

$$
\begin{aligned}
&\|\mathbf{u}\|_{L^{\infty}\left(0, T ;\left(L^{2}\left(\mathcal{B}^{\varepsilon}\right)\right)^{2}\right)} \leq C(\mathbf{f}, \mathbf{g}) \\
&\|\mathbf{D} \mathbf{u}\|_{L^{2}\left(0, T ;\left(L^{2}\left(\mathcal{B}^{\varepsilon}\right)\right)^{4}\right)} \leq C(\mathbf{f}, \mathbf{g}), \\
&\left\|\frac{\partial \mathbf{u}}{\partial t}\right\|_{L^{2}\left(0, T ;\left(L^{2}\left(\mathcal{B}^{\varepsilon}\right)\right)^{2}\right)} \leq\left(\frac{h^{3} E}{12}\right)^{1 / 2} C(\mathbf{f}, \mathbf{g}), \\
&\left\|\frac{\partial d_{ \pm}}{\partial t}\right\|_{L^{\infty}\left(0, T ; L^{2}\left(\Gamma_{ \pm}^{\varepsilon}\right)\right)} \leq C(\mathbf{f}, \mathbf{g}), \\
&\left\|\frac{\partial^{2} d_{ \pm}}{\partial s^{2}}\right\|_{L^{\infty}\left(0, T ; L^{2}\left(\Gamma_{ \pm}^{\varepsilon}\right)\right)} \leq C(\mathbf{f}, \mathbf{g}), \\
&\left\|\frac{\partial^{2} d_{ \pm}}{\partial t^{2}}\right\|_{L^{2}\left(\Gamma_{ \pm}^{\varepsilon} \times(0, T)\right)} \leq\left(\frac{h^{3} E}{12}\right)^{1 / 2} C(\mathbf{f}, \mathbf{g}), \\
&\left\|\frac{\partial^{3} d_{ \pm}}{\partial s^{2} \partial t}\right\|_{L^{\infty}\left(0, T ; L^{2}\left(\Gamma_{ \pm}^{\varepsilon}\right)\right)} \leq\left(\frac{h^{3} E}{12}\right)^{1 / 2} C(\mathbf{f}, \mathbf{g}), \\
&\|\nabla p\|_{L^{2}\left(0, T ;\left(L^{2}\left(B^{\varepsilon}\right)\right)^{2}\right)} \leq\left(\frac{h^{3} E}{12}\right)^{1 / 2} C(\mathbf{f}, \mathbf{g}) .
\end{aligned}
$$

Remark 3.4. If we consider the nonhomogeneous problem (which corresponds to $\boldsymbol{\psi}_{\varepsilon}$ ) we obtain for the corresponding solution the same estimates (3.20) with a different $\mathbf{f}$, but which depends in the same way on $\varepsilon$.

\section{Asymptotic Analysis}

In order to approximate the solution of (2.6), we consider more regular data than in Section 3. We suppose that:

$$
\begin{gathered}
\boldsymbol{\Psi}_{\varepsilon} \in\left(\mathcal{C}^{\infty}\left(\mathcal{B}^{\varepsilon} \times(0, T)\right)\right)^{2}, \\
\mathbf{f} \in\left(\mathcal{C}^{\infty}\left(\mathcal{B}^{\varepsilon} \times(0, T)\right)\right)^{2}, \quad g_{ \pm} \in \mathcal{C}^{\infty}\left(F_{ \pm}^{\varepsilon} \times(0, T)\right), \\
v \in \mathcal{C}^{\infty}\left(\mathcal{B}^{\varepsilon}\right), \\
\exists t^{*}<T \text { such that } \mathbf{f}(x, t)=g_{ \pm}(x, t)=\boldsymbol{\Psi}_{\varepsilon}(x, t)=0 \quad \forall(x, t) \in \mathbb{B}^{\varepsilon} \times\left(0, t^{*}\right),
\end{gathered}
$$


and $\mathbf{f}, g_{ \pm}$and $v$ are chosen as follows:

$$
\begin{aligned}
\mathbf{f}\left(x_{1}, x_{2}, t\right)= \begin{cases}f\left(x_{1}, t\right) \mathbf{e}_{1} & \text { in }\left(\mathbb{B}^{\varepsilon} \cap\left\{x_{1}>\frac{1}{3}\right\}\right) \times(0, T), \\
f\left(x_{2}, t\right) \mathbf{e}_{2} & \text { in }\left(\mathcal{B}^{\varepsilon} \cap\left\{x_{2}>\frac{1}{3}\right\}\right) \times(0, T), \\
0 & \text { in }\left(\mathcal{B}^{\varepsilon} \cap\left\{x_{1} \leq \frac{1}{3}, x_{2} \leq \frac{1}{3}\right\}\right) \times(0, T),\end{cases} \\
g_{ \pm}\left(x_{1}, x_{2}, t\right)= \begin{cases}g_{ \pm}\left(x_{1}, t\right) & \text { on }\left(F_{ \pm}^{\varepsilon} \cap\left\{x_{1}>\frac{1}{3}\right\}\right) \times(0, T), \\
g_{ \pm}\left(x_{2}, t\right) & \text { on }\left(F_{ \pm}^{\varepsilon} \cap\left\{x_{2}>\frac{1}{3}\right\}\right) \times(0, T), \\
0 & \text { on }\left(F_{ \pm}^{\varepsilon} \cap\left\{x_{1} \leq \frac{1}{3}, x_{2} \leq \frac{1}{3}\right\}\right) \times(0, T),\end{cases} \\
\mathcal{v}\left(x_{1}, x_{2}\right)= \begin{cases}v\left(x_{1}\right) & \text { in } \mathbb{B}^{\varepsilon} \cap\left\{\frac{1}{3}<x_{1}<\frac{2}{3}\right\}, \\
\mathcal{v}\left(x_{2}\right) & \text { in } \mathbb{B}^{\varepsilon} \cap\left\{\frac{1}{3}<x_{2}<\frac{2}{3}\right\}, \\
v_{0} & \text { in } \mathbb{B}^{\varepsilon} \cap\left(\left\{x_{1} \leq \frac{1}{3}, x_{2} \leq \frac{1}{3}\right\} \cup\left\{x_{1} \geq \frac{2}{3}\right\} \cup\left\{x_{2} \geq \frac{2}{3}\right\}\right),\end{cases}
\end{aligned}
$$

with $v_{0}$ constant.

\subsection{Construction of the Asymptotic Solution}

In the sequel we introduce the second small parameter $\delta=12 / h^{3} E$ and we take $\delta=\varepsilon^{\gamma}$, with $\gamma \in \mathbb{N}, \gamma \geq 3$. The asymptotic solution approximating the periodic flow in an infinite rectangle (see [1, Section 5]) is modified by using two types of correctors: the first type corresponds to the boundary conditions on $x_{1}=1$ and $x_{2}=1$ and the second one represents the boundary layer functions in a neighborhood of $\left(x_{1}, x_{2}\right)=\mathbf{0}$. So, the asymptotic solution contains the regular part of the solution (which has two terms, due to the form of the flow domain), two boundary layer functions corresponding to $x_{1}=1$ and to $x_{2}=1$, respectively, and the correctors in $\left(x_{1}, x_{2}\right)=\mathbf{0}$. Since the terms of the asymptotic solution should vanish in different parts of the flow domain, we multiply them with the truncation functions defined as follows: $x$, $\eta: \mathbb{R} \mapsto \mathbb{R}, x, \eta \in \mathcal{C}^{\infty}(\mathbb{R})$,

$$
x(\tau)=\left\{\begin{array}{ll}
0, & |\tau|<2, \\
1, & |\tau|>3,
\end{array} \quad \eta(\tau)= \begin{cases}0, & |\tau|<\frac{3}{4} \\
1, & |\tau|>\frac{7}{8}\end{cases}\right.
$$


We construct the asymptotic solution as below:

$$
\begin{aligned}
\widehat{\mathbf{u}}_{a}^{(k)}\left(x_{1}, x_{2}, t\right)= & \mathbf{u}_{1}^{(k)}\left(x_{1}, \frac{x_{2}}{\varepsilon}, t\right) x\left(\frac{x_{1}}{\varepsilon}\right)+\mathbf{u}_{2}^{(k)}\left(\frac{x_{1}}{\varepsilon}, x_{2}, t\right) x\left(\frac{x_{2}}{\varepsilon}\right) \\
& +\mathbf{u}_{b l}^{(k) 1}\left(\frac{x_{1}-1}{\varepsilon}, \frac{x_{2}}{\varepsilon}, t\right) \eta\left(x_{1}\right)+\mathbf{u}_{b l}^{(k) 2}\left(\frac{x_{1}}{\varepsilon}, \frac{x_{2}-1}{\varepsilon}, t\right) \eta\left(x_{2}\right) \\
& +\mathbf{u}_{b l}^{(k) 0}\left(\frac{x_{1}}{\varepsilon}, \frac{x_{2}}{\varepsilon}, t\right) \eta\left(1-x_{1}\right) \eta\left(1-x_{2}\right), \\
\widehat{p}_{a}^{(k)}\left(x_{1}, x_{2}, t\right)= & p_{1}^{(k)}\left(x_{1}, \frac{x_{2}}{\varepsilon}, t\right) x\left(\frac{x_{1}}{\varepsilon}\right)+p_{2}^{(k)}\left(\frac{x_{1}}{\varepsilon}, x_{2}, t\right) x\left(\frac{x_{2}}{\varepsilon}\right) \\
& +p_{b l}^{(k) 1}\left(\frac{x_{1}-1}{\varepsilon}, \frac{x_{2}}{\varepsilon}, t\right) \eta\left(x_{1}\right)+p_{b l}^{(k) 2}\left(\frac{x_{1}}{\varepsilon}, \frac{x_{2}-1}{\varepsilon}, t\right) \eta\left(x_{2}\right) \\
& +p_{b l}^{(k) 0}\left(\frac{x_{1}}{\varepsilon}, \frac{x_{2}}{\varepsilon}, t\right) \eta\left(1-x_{1}\right) \eta\left(1-x_{2}\right), \\
\widehat{d}_{ \pm a}^{(k)}\left(x_{1}, x_{2}, t\right)= & d_{ \pm 1}^{(k)}\left(x_{1}, t\right) \chi\left(\frac{x_{1}}{\varepsilon}\right)+d_{ \pm 2}^{(k)}\left(x_{2}, t\right) \chi\left(\frac{x_{2}}{\varepsilon}\right) \\
& +d_{ \pm b l}^{(k) 1}\left(\frac{x_{1}-1}{\varepsilon}, t\right) \eta\left(x_{1}\right)+d_{ \pm b l}^{(k) 2}\left(\frac{x_{2}-1}{\varepsilon}, t\right) \eta\left(x_{2}\right) \\
& +d_{ \pm b l}^{(k) 0}\left(\frac{x_{1}}{\varepsilon}, \frac{x_{2}}{\varepsilon}, t\right) \eta\left(1-x_{1}\right) \eta\left(1-x_{2}\right) .
\end{aligned}
$$

Due to the definition of the truncation functions, we notice that in $\mathcal{B}^{\varepsilon} \cap\left\{1 / 4 \leq x_{i} \leq 3 / 4\right\}$, that is, in a neighborhood of the region of variable viscosity, the asymptotic solution reduces to its regular part, $\left(\mathbf{u}_{i}^{(k)}, p_{i}^{(k)}, d_{ \pm i}^{(k)}\right)$, in a neighborhood of $\left\{x_{i}=1\right\}$ it reduces to $\left(\mathbf{u}_{i}^{(k)}+\mathbf{u}_{b l}^{(k) i}, p_{i}^{(k)}+\right.$ $\left.p_{b l}^{(k) i}, d_{ \pm i}^{(k)}+d_{ \pm b l}^{(k) i}\right), i=1,2$, while in $D_{\varepsilon}^{r}$ the asymptotic solution is equal to the corrector in $x=$ $\mathbf{0},\left(\mathbf{u}_{b l}^{(k) 0}, p_{b l}^{(k) 0}, d_{ \pm b l}^{(k) 0}\right)$. This means that the regular part of the asymptotic solution (which can be computed and has a very simple expression) represents an approximation for the exact solution in all the flow domain except some neighborhoods of $x_{1}=1, x_{2}=1$ and $x=0$.

We give next the expressions of the three components of the asymptotic solution and we explain their role in this construction.

\subsubsection{The Regular Part of the Asymptotic Solution}

For each rectangle $D_{\varepsilon}^{1}$ and $D_{\varepsilon}^{2}$ we define the corresponding regular part of the asymptotic expansion as in [1]. The regular part corresponding to $D_{\varepsilon}^{1}$ has the expression:

$$
\mathbf{u}_{1}^{(k)}\left(x_{1}, \frac{x_{2}}{\varepsilon}, t\right)=\sum_{j=0}^{k} \varepsilon^{j+2} u_{1, j}^{1}\left(x_{1}, \frac{x_{2}}{\varepsilon}, t\right) \mathbf{e}_{1}+\sum_{j=0}^{k} \varepsilon^{j+3} u_{2, j}^{1}\left(x_{1}, \frac{x_{2}}{\varepsilon}, t\right) \mathbf{e}_{2},
$$




$$
\begin{aligned}
p_{1}^{(k)}\left(x_{1}, \frac{x_{2}}{\varepsilon}, t\right) & =\sum_{j=0}^{k} \varepsilon^{j+1} p_{j}^{1}\left(x_{1}, \frac{x_{2}}{\varepsilon}, t\right)+\sum_{j=0}^{k} \varepsilon^{j} q_{j}^{1}\left(x_{1}, t\right), \\
d_{ \pm 1}^{(k)}\left(x_{1}, t\right) & =\sum_{j=0}^{k} \varepsilon^{j+\gamma} d_{( \pm) j}^{1}\left(x_{1}, t\right) .
\end{aligned}
$$

It represents the solution of the problem $(2.6)_{1,2,3,11}$ set in the infinite rectangle in $O x_{1}$ direction, $(-\infty, \infty) \times(-\varepsilon, \varepsilon)$; the functions which appear in (4.5) can be computed explicitly and represent a good approximation of the exact solution of (2.6) in a neighborhood of the region with variable viscosity of $D_{\varepsilon}^{1}$ (as we will prove in the last section). ing to $D_{\varepsilon}^{2}$.

In a similar way we introduce the regular part of the asymptotic solution correspond-

Hence, the sum of the first two terms of the asymptotic solution represents a good approximation of the exact solution of (2.6) in some neighborhood of the region of variable viscosity of the flow domain, but it is not close to it on the inflow/outflow boundaries and in $D_{\varepsilon}^{r}$. Since the purpose of the asymptotic construction is to approximate the exact solution with a small error between the exact and asymptotic solution, we modify and complete the regular part by two types of boundary layer correctors.

\subsubsection{The Boundary Layer Correctors for $x_{1}=1$ and $x_{2}=1$}

These boundary layer functions are introduced in order to repair the traces of the regular part of the asymptotic solution on $x_{1}=1$ and $x_{2}=1$. They are given by:

$$
\begin{gathered}
\mathbf{u}_{b l}^{(k) i}\left(\frac{x}{\varepsilon}, t\right)=\sum_{j=0}^{k} \varepsilon^{j+2} \mathbf{u}_{j}^{(i)}\left(\frac{x}{\varepsilon}, t\right), \\
p_{b l}^{(k) i}\left(\frac{x}{\varepsilon}, t\right)=\sum_{j=0}^{k} \varepsilon^{j+1} p_{j}^{(i)}\left(\frac{x}{\varepsilon}, t\right), \\
d_{ \pm b l}^{(k) i}\left(\frac{x_{i}}{\varepsilon}, t\right)=\sum_{j=0}^{k} \varepsilon^{j+\gamma} d_{j}^{(i)}\left(\frac{x_{i}}{\varepsilon}, t\right), \quad i \in\{1,2\} .
\end{gathered}
$$

The corrector with $i=1$ corresponds to the end $x_{1}=1$ and that with $i=2$ corresponds to $x_{2}=1$.

From the definition of the truncation functions and of the asymptotic solution we notice that the influence of each corrector defined above is significant only near the corresponding end of the flow domain.

\subsubsection{The Boundary Layer Corrector in $x=0$}

This corrector is necessary in order to realize the junction between the two parts of the asymptotic solution, corresponding to the two branches of the flow domain and to obtain the 
conditions on the rigid boundaries of the domain. The expressions of the correctors in $x=0$ are given by:

$$
\begin{aligned}
& \mathbf{u}_{b l}^{(k) 0}\left(\frac{x}{\varepsilon}, t\right)=\sum_{j=-1}^{k} \varepsilon^{j+2} \mathbf{u}_{j}^{(0)}\left(\frac{x}{\varepsilon}, t\right), \\
& p_{b l}^{(k) 0}\left(\frac{x}{\varepsilon}, t\right)=\sum_{j=-1}^{k} \varepsilon^{j+1} p_{j}^{(0)}\left(\frac{x}{\varepsilon}, t\right), \\
& d_{ \pm b l}^{(k) 0}\left(\frac{x}{\varepsilon}, t\right)=\sum_{j=0}^{k} \varepsilon^{j+\gamma} d_{( \pm) j}^{(0)}\left(\frac{x}{\varepsilon}, t\right) .
\end{aligned}
$$

From the definition of the truncation function $\eta$ it follows that the corrector in $x=0$ appears in the expression of the asymptotic solution (4.4) only in $\mathbb{B}^{\varepsilon} \cap\left\{x_{1} \leq 1 / 4, x_{2} \leq 1 / 4\right\}$, which represents a neighborhood of $D_{\varepsilon}^{r}$ with constant viscosity and where the forces are equal to zero.

Remark 4.1. The boundary layer method is close to the well-known method of matching of asymptotic expansions (see [14]). However there is a difference between these two methods. In the boundary layer method the regular expansion is reexpanded with respect to the fast variable (as in the matching method), but then the new expansion is multiplied by a cutting function. This product being inserted into the equation gives a discrepancy in the right-hand side which is then compensated by an appropriate boundary layer corrector. So, we get finally a unique expression for the asymptotic approximation in each point of the domain. In the matching approach the reexpanded regular ansatz (outer expansion) coexists with the so-called inner expansion defined near the boundary, so that there are several overlapping expressions for an asymptotic approximation in different parts of the domain. We emphasize that this difference is not too important and corresponds more to the form of presentation of the result.

\subsection{The Determination of the Asymptotic Solution}

This subsection is devoted to the resolution of the problems satisfied by the three different components of the asymptotic solution presented in the previous subsection. Since some computations are different with respect to the values of $\gamma$, we will analyse the problems and the order of solving them for $\gamma>3$ and for $\gamma=3$. Moreover, in each case we will specify the leading term of the asymptotic expansion.

We begin the approach with the problems for the boundary layer correctors corresponding to $x_{1}=1$ and $x_{2}=1$ since the study of these problems is the same both for $\gamma>3$ and for $\gamma=3$. To fix the ideas, we obtain in the sequel the problems for the corrector corresponding to the end $x_{1}=1$. As we noticed before, the term containing this corrector is not equal to zero only in a neighborhood of the boundary $x_{1}=1$. So, the problems and the other relations corresponding to this corrector are obtained substituting the asymptotic solution in $(2.6)_{1,2,3,6,7,11}$. We obtain two separate problems: one for the velocity-pressure correctors and the other one for the displacements correctors. 
Since in this neighborhood the viscosity is constant, the problem for $\left(\mathbf{u}_{j}^{(1)}, p_{j}^{(1)}\right)$ has constant coefficients. Denoting by $\Pi^{1}$ the semi-infinite rectangle $(-\infty, 0) \times(-1,1)$ and imposing for the velocity and for the pressure the condition of decay at $-\infty$, we obtain for $\left(\mathbf{u}_{j}^{(1)}, p_{j}^{(1)}\right)$ the problem:

$$
\begin{gathered}
-v_{0} \Delta_{\xi \xi} \mathbf{u}_{j}^{(1)}+\nabla_{\xi} p_{j}^{(1)}=-\rho_{f} \frac{\partial \mathbf{u}_{j-2}^{(1)}}{\partial t} \text { in } \Pi^{1} \times(0, T), \\
\operatorname{div}_{\xi} \mathbf{u}_{j}^{(1)}=0 \quad \text { in } \Pi^{1} \times(0, T), \\
\mathbf{u}_{j}^{(1)}\left(\xi_{1}, \pm 1, t\right)=\frac{\partial d_{( \pm) j+2-\gamma}^{(1)}}{\partial t}\left(\xi_{1}, t\right) \mathbf{e}_{2} \text { in }(-\infty, 0) \times(0, T), \\
\mathbf{u}_{j}^{(1)}\left(0, \xi_{2}, t\right)=\psi\left(\xi_{2}, t\right) \delta_{j 0} \mathbf{e}_{1}-u_{1, j}^{1}\left(1, \xi_{2}, t\right) \mathbf{e}_{1}-u_{2, j-1}^{1}\left(1, \xi_{2}, t\right) \mathbf{e}_{2} \quad \text { in }(-1,1) \times(0, T), \\
\mathbf{u}_{j}^{(1)} \longrightarrow \mathbf{0 ,} p_{j}^{(1)} \longrightarrow 0, \quad \text { uniformly when } \xi_{1} \longrightarrow-\infty .
\end{gathered}
$$

The compatibility condition for (4.8) reads:

$$
\int_{-1}^{1} u_{1, j}^{1}\left(1, \xi_{2}, t\right) \mathrm{d} \xi_{2}=\frac{\mathrm{d}}{\mathrm{d} t} \int_{-\infty}^{0}\left(d_{(+) j+2-\gamma}^{(1)}-d_{(-) j+2-\gamma}^{(1)}\right)\left(\xi_{1}, t\right) \mathrm{d} \xi_{1}+\delta_{j 0} \int_{-1}^{1} \psi\left(\xi_{2}, t\right) \mathrm{d} \xi_{2} .
$$

For any $\gamma \geq 3$ the right-hand side of $(4.8)_{3}$ is known; so the boundary layer correctors for the velocity and for the pressure corresponding to $x_{1}=1$ are uniquely determined from (4.8) (see [2, Section 4]). The condition (4.9) represents a relation for the regular part of the asymptotic solution.

The boundary layer correctors for the displacements exponentially stabilizing to zero at $-\infty$ are obtained as the unique solution of the following problems:

$$
\begin{aligned}
& \frac{\partial^{4} d_{( \pm) j}^{(1)}}{\partial \xi_{1}^{4}}=-\rho h \frac{\partial^{2} d_{( \pm) j-4-\gamma}^{(1)}}{\partial t^{2}}-\mu \frac{\partial^{5} d_{( \pm) j-\gamma}^{(1)}}{\partial \xi_{1}^{4} \partial t} \pm p_{j-\left.5\right|_{\xi_{2}= \pm 1} ^{(1)}}^{4} \quad \text { in }(-\infty, 0) \times(0, T), \\
& \frac{\partial^{a} d_{( \pm) j}^{(1)}}{\partial \xi_{1}^{a}} \longrightarrow 0, \quad \text { uniformly, when only } \xi_{1} \longrightarrow-\infty, a \in\{0,1,2,3\} .
\end{aligned}
$$

Since at the step $j$ the problem (4.10) gives both $d_{( \pm) j}^{(1)}$ and $d_{( \pm) j+1}^{(1)}$, introducing the asymptotic solution into (2.6) t we obtain two boundary conditions for the regular part of the asymptotic solution for the displacements:

$$
\begin{gathered}
d_{( \pm) j}^{1}(1, t)=-d_{( \pm) j}^{(1)}(0, t), \\
\frac{\partial d_{( \pm) j}^{1}}{\partial x_{1}}(1, t)=-\frac{\partial d_{( \pm) j+1}^{(1)}}{\partial \xi_{1}}(0, t) .
\end{gathered}
$$


In a similar way, we obtain the boundary layer correctors corresponding to the end $x_{2}=1$. The boundary layers for the velocity-pressure are defined on $\Pi^{2} \times(0, T)$, with $\Pi^{2}=$ $(-1,1) \times(-\infty, 0)$, and the boundary layers for the displacements are defined also on $(-\infty, 0) \times$ $(0, T)$.

We study next the problems for the regular parts of the asymptotic solution. The results are obtained for the regular part corresponding to $D_{\varepsilon}^{1}$; the regular part corresponding to $D_{\varepsilon}^{2}$ may be obtained from the previous with some obvious changes.

Introducing (4.5) into (2.6) $)_{1,2,3,11}$ and collecting together the terms of the same order with respect to $\varepsilon$ we are leaded to consider the following problem for $\left(u_{1, j}^{1}, u_{2, j}^{1}, p_{j}^{1}, q_{j}^{1}, d_{( \pm) j}^{1}\right)$ :

$$
\begin{gathered}
-v\left(x_{1}\right) \frac{\partial^{2} u_{1, j}^{1}}{\partial \xi_{2}^{2}}+\frac{\partial q_{j}^{1}}{\partial x_{1}}=f \delta_{j 0}-\rho_{f} \frac{\partial u_{1, j-2}^{1}}{\partial t}+2 \frac{\partial}{\partial x_{1}}\left(v\left(x_{1}\right) \frac{\partial u_{1, j-2}^{1}}{\partial x_{1}}\right)+v\left(x_{1}\right) \frac{\partial^{2} u_{2, j-2}^{1}}{\partial \xi_{2} \partial x_{1}}-\frac{\partial p_{j-1}^{1}}{\partial x_{1}}, \\
\frac{\partial p_{j}^{1}}{\partial \xi_{2}}=-\rho_{f} \frac{\partial u_{2, j-3}^{1}}{\partial t}+\frac{\partial}{\partial x_{1}}\left(v\left(x_{1}\right) \frac{\partial u_{2, j-3}^{1}}{\partial x_{1}}\right)+\frac{\partial}{\partial x_{1}}\left(v\left(x_{1}\right) \frac{\partial u_{1, j-1}^{1}}{\partial \xi_{2}}\right)+2 v\left(x_{1}\right) \frac{\partial^{2} u_{2, j-1}^{1}}{\partial \xi_{2}^{2}}, \\
\frac{\partial u_{1, j}^{1}}{\partial x_{1}}+\frac{\partial u_{2, j}^{1}}{\partial \xi_{2}}=0 \text { in }(0,1) \times(-1,1) \times(0, T),
\end{gathered}
$$

$\frac{\partial^{4} d_{(+) j}^{1}}{\partial x_{1}^{4}}-q_{j}^{1}=g_{+} \delta_{j 0}+\left.p_{j-1}^{1}\right|_{\xi_{2}=1}-\rho h \frac{\partial^{2} d_{(+) j-\gamma}^{1}}{\partial t^{2}}-\mu \frac{\partial^{5} d_{(+) j-\gamma}^{1}}{\partial x_{1}^{4} \partial t} \quad$ in $(0,1) \times\{-1\} \times(0, T)$,

$$
\begin{gathered}
\frac{\partial^{4} d_{(-) j}^{1}}{\partial x_{1}^{4}}+q_{j}^{1}=g_{-} \delta_{j 0}-p_{j-1}^{1}-\rho h \frac{\partial^{2} d_{(-) j-\gamma}^{1}-\gamma}{\partial t^{2}}-\mu \frac{\partial^{5} d_{(-) j-\gamma}^{1}}{\partial x_{1}^{4} \partial t} \quad \text { in }(0,1) \times\{1\} \times(0, T), \\
\mathbf{u}_{j}^{1}\left(x_{1}, \pm 1, t\right)=\frac{\partial d_{( \pm) j-\gamma+3}^{1}}{\partial t}\left(x_{1}, t\right) \mathbf{e}_{2} \quad \text { in }(0,1) \times\{ \pm 1\} \times(0, T) .
\end{gathered}
$$

The two cases, $\gamma>3$ and $\gamma=3$, appear because of the last relation of the previous system. We can see, indeed, that for $\gamma>3$ the unknown of this relation is $\mathbf{u}_{j}^{1}$, while for $\gamma=3,(4.12)_{6}$ contains two unknowns. From this point, the computations are different with respect to the values of $\gamma$.

We introduce the functions:

$$
N_{1}\left(\xi_{2}\right)=\frac{1}{2}\left(\xi_{2}^{2}-1\right), \quad N_{2}\left(\xi_{2}\right)=\int_{-1}^{\xi_{2}} N_{1}(\tau) \mathrm{d} \tau
$$

with the properties: $N_{1}^{\prime \prime}=1, N_{1}( \pm 1)=0$ and $N_{2}(1)=-2 / 3$. 
We also use the notations:

$$
\begin{gathered}
U_{j-1}^{1}\left(x_{1}, \xi_{2}, t\right)=\rho_{f} \frac{\partial u_{1, j-2}^{1}}{\partial t}-2 \frac{\partial}{\partial x_{1}}\left(v\left(x_{1}\right) \frac{\partial u_{1, j-2}^{1}}{\partial x_{1}}\right)-v\left(x_{1}\right) \frac{\partial^{2} u_{2, j-2}^{1}}{\partial \xi_{2} \partial x_{1}}+\frac{\partial p_{j-1}^{1}}{\partial x_{1}}, \\
\Phi_{( \pm) j-1}^{1}\left(x_{1}, t\right)= \pm p_{j-1}^{1}-\rho h \frac{\partial^{2} d_{( \pm) j-\gamma}^{1}}{\partial t^{2}}-\mu \frac{\partial^{5} d_{( \pm) j-\gamma}^{1}}{\partial x_{1}^{4} \partial t}, \\
D^{-1}: F \longrightarrow \int_{-1}^{\xi_{2}} F\left(x_{1}, \tau\right) \mathrm{d} \tau, \\
D^{-2}: F \longrightarrow \int_{-1}^{\xi_{2}} \int_{-1}^{\theta} F\left(x_{1}, \tau\right) \mathrm{d} \tau \mathrm{d} \theta-\frac{1}{2}\left(\xi_{2}+1\right) \int_{-1}^{1} \int_{-1}^{\theta} F\left(x_{1}, \tau\right) \mathrm{d} \tau \mathrm{d} \theta, \\
I_{x_{1}}^{-k}: F \longrightarrow \int_{0}^{x_{1}} \int_{0}^{a_{k-1}} \cdots \int_{0}^{a_{1}} F\left(s, \xi_{2}, t\right) \mathrm{d} s, \\
J_{x_{1}}: F \longrightarrow I_{x_{1}}^{-4}(F)+x_{1}^{3}\left(2 \int_{0}^{1} I_{x_{1}}^{-3}(F)-\int_{0}^{1} I_{x_{1}}^{-2}(F)\right)-x^{2}\left(3 \int_{0}^{1} I_{x_{1}}^{-3}(F)-\int_{0}^{1} I_{x_{1}}^{-2}(F)\right) .
\end{gathered}
$$

\subsubsection{The Order of Solving the Problems for $\gamma>3$}

The regular part corresponding to $D_{\varepsilon}^{1}$ is computed by integrating (4.12), as stated below.

Proposition 4.2. The unknowns $\mathbf{u}_{j}^{1}, q_{j}^{1}, p_{j}^{1}, d_{( \pm) j}^{1}$ are determined from (4.12), up to nine functions of $t$.

Proof. Integrating twice $(4.12)_{1}$ from -1 to $\xi_{2}$ and using the boundary conditions $(4.12)_{6}$ we get:

$$
u_{1, j}^{1}\left(x_{1}, \xi_{2}, t\right)=\frac{1}{v\left(x_{1}\right)} D^{-2}\left(U_{j-1}^{1}\right)+\frac{1}{v\left(x_{1}\right)}\left(\frac{\partial q_{j}^{1}}{\partial x_{1}}-f \delta_{j 0}\right) N_{1}\left(\xi_{2}\right)
$$

which contains as unknowns $u_{1, j}^{1}$ and $q_{j}^{1}$. The other functions contained by this relation are either known from previous computations or equal to zero.

We integrate next the incompressibility condition $(4.12)_{3}$ with respect to $\xi_{2}$ with the boundary condition $(4.12)_{6}$ for $\xi_{2}=-1$ and we obtain

$$
\begin{aligned}
u_{2, j}^{1}\left(x_{1}, \xi_{2}, t\right)= & \frac{\partial d_{(-) j-\gamma+3}^{1}}{\partial t}-\frac{\partial}{\partial x_{1}}\left(\frac{1}{v\left(x_{1}\right)}\left(\frac{\partial q_{j}^{1}}{\partial x_{1}}-f \delta_{j 0}\right)\right) N_{2}\left(\xi_{2}\right) \\
& -D^{-1} D^{-2}\left(\frac{\partial}{\partial x_{1}}\left(\frac{1}{v\left(x_{1}\right)} U_{j-1}^{1}\right)\right) .
\end{aligned}
$$

The previous two relations give the components of the velocity $\mathbf{u}_{j}^{1}$ with respect to $q_{j}^{1}$. 
The pressure approximations are determined from (4.12) 2 , supposing that the integration functions, depending on $x_{1}, t$ are equal to zero, since we consider that any function depending only on $x_{1}, t$ could be contained in $q_{j+1}^{1}$.

$$
p_{j}^{1}=D^{-1}\left(-\rho_{f} \frac{\partial u_{2, j-3}^{1}}{\partial t}+\frac{\partial}{\partial x_{1}}\left(v\left(x_{1}\right) \frac{\partial u_{2, j-3}^{1}}{\partial x_{1}}\right)+\frac{\partial}{\partial x_{1}}\left(v\left(x_{1}\right) \frac{\partial u_{1, j-1}^{1}}{\partial \xi_{2}}\right)+2 v\left(x_{1}\right) \frac{\partial^{2} u_{2, j-1}^{1}}{\partial \xi_{2}^{2}}\right)
$$

Taking $\xi_{2}=1$ in (4.16) and using the boundary condition $(4.12)_{6}$ for $\xi_{2}=1$ we obtain the following second order differential equation for the function $q_{j}^{1}$ :

$$
\begin{aligned}
\frac{\partial}{\partial x_{1}}\left(\frac{1}{v\left(x_{1}\right)}\left(\frac{\partial q_{j}^{1}}{\partial x_{1}}-f \delta_{j 0}\right)\right)= & \frac{3}{2} \frac{\partial}{\partial t}\left(d_{(+) j-\gamma+3}^{1}-d_{(-) j-\gamma+3}^{1}\right) \\
& +\frac{3}{2} \int_{-1}^{1} D^{-2}\left(\frac{\partial}{\partial x_{1}}\left(\frac{1}{v\left(x_{1}\right)} U_{j-1}^{1}\right)\right) d \xi_{2} .
\end{aligned}
$$

Integrating (4.18) from $x_{1}$ to 1 , we express $\partial q_{j}^{1} / \partial x_{1}$ by means of $\left(\partial q_{j}^{1} / \partial x_{1}\right)(1, t)$, which represents the only unknown of this expression. This function of $t$ is obtained as follows: we take $x_{1}=1$ in (4.15) and we introduce the result into (4.9). Hence, we determined the expression of $\partial q_{j}^{1} / \partial x_{1}$ in $(0,1) \times(0, T)$, which is:

$$
\begin{aligned}
\frac{1}{v\left(x_{1}\right)}\left(\frac{\partial q_{j}^{1}}{\partial x_{1}}-f \delta_{j 0}\right)= & -\frac{3}{2} \delta_{j 0} \int_{-1}^{1} \psi\left(\xi_{2}, t\right) \mathrm{d} \xi_{2}-\frac{3}{2} \frac{\mathrm{d}}{\mathrm{d} t} \int_{-\infty}^{0}\left(d_{(+) j+2-\gamma}^{(1)}-d_{(-) j+2-\gamma}^{(1)}\right)\left(\xi_{1}, t\right) \mathrm{d} \xi_{1} \\
& -\frac{3}{2} \frac{\partial}{\partial t} \int_{x_{1}}^{1}\left(d_{(+) j-\gamma+3}^{1}-d_{(-) j-\gamma+3}^{1}\right)(s, t) \mathrm{d} s+\frac{3}{2} \int_{-1}^{1} D^{-2}\left(\frac{1}{v\left(x_{1}\right)} U_{j-1}^{1}\right) \mathrm{d} \xi_{2} .
\end{aligned}
$$

Introducing (4.19) into (4.15) and (4.18) into (4.16) we determine $\mathbf{u}_{j}^{1}$ in $(0,1) \times(-1,1) \times(0, T)$.

We integrate next $v\left(x_{1}\right) \cdot(4.19)$ from 0 to $x_{1}$ and we get $q_{j}^{1}$ determined up to the function of $t, q_{j}^{1}(0, t)$ :

$$
\begin{aligned}
q_{j}^{1}\left(x_{1}, t\right)= & \delta_{j 0} \int_{0}^{x_{1}} f(s, t) \mathrm{d} s-\frac{3}{2} \int_{0}^{x_{1}} v(\theta) \int_{\theta}^{1} \frac{\partial}{\partial t}\left(d_{(+) j-\gamma+3}^{1}-d_{(-) j-\gamma+3}^{1}\right)(s, t) \mathrm{d} s \mathrm{~d} \theta \\
& -\frac{3}{2}\left(\frac{\mathrm{d}}{\mathrm{d} t} \int_{-\infty}^{0}\left(d_{(+) j+2-\gamma}^{(1)}-d_{(-) j+2-\gamma}^{(1)}\right)\left(\xi_{1}, t\right) \mathrm{d} \xi_{1}+\delta_{j 0} \int_{-1}^{1} \psi\left(\xi_{2}, t\right) \mathrm{d} \xi_{2}\right)\left(\int_{0}^{x_{1}} v(s) \mathrm{d} s\right) \\
& +\frac{3}{2} \int_{0}^{x_{1}} \int_{-1}^{1} D^{-2}\left(U_{j-1}^{1}\left(s, \xi_{2}, t\right)\right) \mathrm{d} \xi_{2} \mathrm{~d} s+q_{j}^{1}(0, t) .
\end{aligned}
$$


The functions $d_{( \pm) j}^{1}$ satisfy the fourth-order differential equations:

$$
\frac{\partial^{4} d_{( \pm) j}^{1}}{\partial x_{1}^{4}}= \pm q_{j}^{1}+g_{ \pm} \delta_{j 0}+\Phi_{( \pm) j-1}^{1}
$$

with $q_{j}^{1}$ given by (4.20). Writting $q_{j}^{1}$ as $Q_{j}^{1}\left(x_{1}, t\right)+q_{j}^{1}(0, t)$ and integrating four times (4.21) with respect to $x_{1}$ we obtain the following expressions for $d_{( \pm) j}^{1}$ :

$$
\begin{aligned}
& d_{(+) j}^{1}\left(x_{1}, t\right)=d_{(+) j}^{1}(0, t)\left(1-3 x_{1}{ }^{2}+2 x_{1}^{3}\right)+\frac{\partial d_{(+) j}^{1}}{\partial x_{1}}(0, t)\left(x_{1}-2 x_{1}^{2}+x_{1}^{3}\right) \\
& +d_{(+) j}^{1}(1, t)\left(3 x_{1}^{2}-2 x_{1}^{3}\right)+\frac{\partial d_{(+) j}^{1}}{\partial x_{1}}(1, t)\left(-x_{1}{ }^{2}+x_{1}^{3}\right) \\
& +q_{j}^{1}(0, t)\left(\frac{x_{1}{ }^{2}}{24}-\frac{x_{1}{ }^{3}}{12}+\frac{x_{1}^{4}}{24}\right)+J_{x_{1}}\left(Q_{j}^{1}\right)+J_{x_{1}}\left(g_{+}\right) \delta_{j 0}+J_{x_{1}}\left(\Phi_{(+) j-1}^{1}\right), \\
& d_{(-) j}^{1}\left(x_{1}, t\right)=d_{(-) j}^{1}(0, t)\left(1-3 x_{1}^{2}+2 x_{1}^{3}\right)+\frac{\partial d_{(-) j}^{1}}{\partial x_{1}}(0, t)\left(x_{1}-2 x_{1}{ }^{2}+x_{1}{ }^{3}\right) \\
& +d_{(-) j}^{1}(1, t)\left(3 x_{1}^{2}-2 x_{1}^{3}\right)+\frac{\partial d_{(-) j}^{1}}{\partial x_{1}}(1, t)\left(-x_{1}^{2}+x_{1}^{3}\right) \\
& -q_{j}^{1}(0, t)\left(\frac{x_{1}{ }^{2}}{24}-\frac{x_{1}^{3}}{12}+\frac{x_{1}^{4}}{24}\right)-J_{x_{1}}\left(Q_{j}^{1}\right)+J_{x_{1}}\left(g_{-}\right) \delta_{j 0}+J_{x_{1}}\left(\Phi_{(-) j-1}^{1}\right) .
\end{aligned}
$$

Hence, the regular part of the asymptotic solution corresponding to $D_{\varepsilon}^{1}$ is determined up to the functions $q_{j}^{1}(0, t), d_{( \pm) j}^{1}(0, t),\left(\partial d_{( \pm) j}^{1} / \partial x_{1}\right)(0, t), d_{( \pm) j}^{1}(1, t),\left(\partial d_{( \pm) j}^{1} / \partial x_{1}\right)(1, t)$, which achieves the proof.

In a similar way we express the regular part of the asymptotic solution corresponding to $D_{\varepsilon}^{2}$ depending on 9 unknown functions of $t$.

We continue the construction with the problems for the corrector in $x=0$. As we previously said, the term of the asymptotic solution containing this corrector is not equal to zero only in a neighborhood of $D_{\varepsilon}^{r}$. In this neighborhood, the expression of the asymptotic solution reduces to $\left(\mathbf{u}_{1}^{(k)}\left(x_{1}, \xi_{2}, t\right) \chi\left(\xi_{1}\right)+\mathbf{u}_{2}^{(k)}\left(\xi_{1}, x_{2}, t\right) \chi\left(\xi_{2}\right)+\mathbf{u}_{b l}^{(k) 0}(\xi, t), p_{1}^{(k)}\left(x_{1}, \xi_{2}, t\right) \chi\left(\xi_{1}\right)+\right.$ $\left.p_{2}^{(k)}\left(\xi_{1}, x_{2}, t\right) \chi\left(\xi_{2}\right)+p_{b l}^{(k) 0}(\xi, t), d_{ \pm 1}^{(k)}\left(x_{1}, t\right) \chi\left(\xi_{1}\right)+d_{ \pm 2}^{(k)}\left(x_{2}, t\right) \chi\left(\xi_{2}\right)+d_{ \pm b l}^{(k) 0}(\xi, t)\right)$. For obtaining the problems satisfied by the correctors in $x=0$, we introduce the previous expression of the asymptotic solution in $(2.6)_{1,2,3,4,5,6,11}$, with $\boldsymbol{v}=v_{0}$ and $\mathbf{f}=\mathbf{g}=\mathbf{0}$ in $(2.6)_{1,3}$; for derivating the terms which contain the two types of variables $x_{i}$ and $\xi_{i}$ we proceed as follows: we replace $\partial / \partial x_{i}$ by $(1 / \varepsilon)\left(\partial / \partial \xi_{i}\right)$, we replace $x_{i}$ by $\varepsilon \xi_{i}$ and we expand the functions as a Taylor expansion with respect to $x_{i}=\varepsilon \xi_{i}$. We introduce the notations $B=D^{r} \cup\left\{\left(\xi_{1}, \xi_{2}\right): \xi_{1} \geq 2, \xi_{2} \in\right.$ $(-1,1)\} \cup\left\{\left(\xi_{1}, \xi_{2}\right): \xi_{1} \in(-1,1), \xi_{2} \geq 2\right\}, \widehat{A_{ \pm} B_{ \pm}}=(1 / \varepsilon) \widehat{A_{ \pm}^{\varepsilon} B_{ \pm}^{\varepsilon}}$ and we obtain for $\left(\mathbf{u}_{j}^{(0)}, p_{j}^{(0)}\right)$ 
the following nondivergence free problem:

$$
\begin{aligned}
&-v_{0} \Delta_{\xi \xi} \mathbf{u}_{j}^{(0)}+\nabla_{\xi} p_{j}^{(0)}=-\rho_{f} \frac{\partial \mathbf{u}_{j-2}^{(0)}}{\partial t}+\mathbf{F}_{j} \\
&+\left(1-\chi\left(\xi_{1}\right)\right)_{\xi_{1} 1}^{\prime} q_{j+1}^{1}(0, t) \mathbf{e}_{1} \\
&+\left(1-\chi\left(\xi_{2}\right)\right)_{\xi_{2}}^{\prime} q_{j+1}^{2}(0, t) \mathbf{e}_{2} \text { in } \mathcal{B}, \\
& \operatorname{div} \mathbf{u}_{j}^{(0)}= \tilde{\theta}_{j} \quad \text { in } \mathcal{B}, \\
& \mathbf{u}_{j}^{(0)}= \mathbf{0} \quad \text { on } \widehat{A_{+} B_{+} \cup A_{-} B_{-},} \\
& \mathbf{u}_{j}^{(0)}\left(\xi_{1}, \pm 1, t\right)= \frac{\partial d_{( \pm) j+2-\gamma}^{(0)}}{\partial t}\left(\xi_{1}, \pm 1, t\right) \mathbf{e}_{2} \quad \text { for } \xi_{1} \geq 2, \\
& \mathbf{u}_{j}^{(0)}\left( \pm 1, \xi_{2}, t\right)= \frac{\partial d_{( \pm) j+2-\gamma}^{(0)}}{\partial t}\left( \pm 1, \xi_{2}, t\right) \mathbf{e}_{1} \quad \text { for } \xi_{2} \geq 2, \\
& \mathbf{u}_{j}^{(0)} \longrightarrow \mathbf{0} \quad \text { uniformly when } \xi_{1} \longrightarrow \infty \\
& \mathbf{u}_{j}^{(0)} \longrightarrow \mathbf{0} \text { uniformly when } \xi_{2} \longrightarrow \infty,
\end{aligned}
$$

for $j \geq 0$ and

$$
\begin{aligned}
& -v_{0} \Delta_{\xi \xi} \mathbf{u}_{-1}^{(0)}+\nabla_{\xi} p_{-1}^{(0)}=\left(1-\chi\left(\xi_{1}\right)\right)_{\xi_{1}}^{\prime} q_{0}^{1}(0, t) \mathbf{e}_{1}+\left(1-\chi\left(\xi_{2}\right)\right)_{\xi_{2}}^{\prime} q_{0}^{2}(0, t) \mathbf{e}_{2} \quad \text { in } 乃, \\
& \operatorname{div} \mathbf{u}_{-1}^{(0)}=0 \text { in } \boldsymbol{B}, \\
& \mathbf{u}_{-1}^{(0)}=\mathbf{0} \text { on } \widehat{A_{+} B_{+}} \cup \widehat{A_{-} B_{-}} \text {, } \\
& \mathbf{u}_{-1}^{(0)}\left(\xi_{1}, \pm 1, t\right)=\mathbf{0} \text { for } \xi_{1} \geq 2 \text {, } \\
& \mathbf{u}_{-1}^{(0)}\left( \pm 1, \xi_{2}, t\right)=\mathbf{0} \quad \text { for } \xi_{2} \geq 2 \text {, } \\
& \mathbf{u}_{-1}^{(0)} \longrightarrow \mathbf{0} \text { uniformly when } \xi_{1} \longrightarrow \infty \text {, } \\
& \mathbf{u}_{-1}^{(0)} \longrightarrow \mathbf{0} \text { uniformly when } \xi_{2} \longrightarrow \infty \text {. }
\end{aligned}
$$

Unlike the problem (4.8) which give the correctors for $x_{1}=1$, the problems (4.23), (4.24) have unknown right hand sides. The functions $\mathbf{F}_{j}$ are known, but $q_{j+1}^{1}(0, t)$ and $q_{j+1}^{2}(0, t)$ are elements from the next approximation, so they are unknown. The function $\tilde{\theta}_{j}$ is given by:

$$
\begin{aligned}
\tilde{\theta}_{j}=-\operatorname{div}_{\xi}\left(\chi\left(\xi_{1}\right) \sum_{l=0}^{j} \frac{\xi_{1}^{l}}{l !}\left(\frac{\partial^{l} u_{1, j-l}^{1}}{\partial x_{1}{ }^{l}}\left(0, \xi_{2}, t\right) \mathbf{e}_{1}+\frac{\partial^{l} u_{2, j-1-l}^{1}}{\partial x_{1}{ }^{l}}\left(0, \xi_{2}, t\right) \mathbf{e}_{2}\right)\right. \\
\left.+\chi\left(\xi_{2}\right) \sum_{l=0}^{j} \frac{\xi_{2}^{l}}{l !}\left(\frac{\partial^{l} u_{1, j-1-l}^{2}}{\partial x_{2}{ }^{l}}\left(\xi_{1}, 0, t\right) \mathbf{e}_{1}+\frac{\partial^{l} u_{2, j-l}^{2}}{\partial x_{2}{ }^{l}}\left(\xi_{1}, 0, t\right) \mathbf{e}_{2}\right)\right),
\end{aligned}
$$

and it has to satisfy a compatibility condition. 
The problem (4.24) has the unique solution (with the pressure unique up to an additive function of $t$ )

$$
\begin{aligned}
& \mathbf{u}_{-1}^{(0)}=\mathbf{0}, \\
& p_{-1}^{(0)}= \begin{cases}\left(1-\chi\left(\xi_{1}\right)\right) q_{0}^{1}(0, t) & \text { for } \xi_{1} \geq 2, \\
q_{0}^{1}(0, t)=q_{0}^{2}(0, t) & \text { in } D_{r} \\
\left(1-\chi\left(\xi_{2}\right)\right) q_{0}^{2}(0, t) & \text { for } \xi_{2} \geq 2\end{cases}
\end{aligned}
$$

We obtain next the problems for the correctors in $x=\mathbf{0}$ corresponding to the displacements. We first notice that

$$
d_{( \pm) j}^{(0)}=0 \quad \text { on } \widehat{A_{ \pm} B_{ \pm}}
$$

Let us denote by $\widehat{d}_{( \pm) j-1}^{(0)}$ the unique solution of

$$
\begin{aligned}
\frac{\partial^{4} \widehat{d}_{( \pm) j-1}^{(0)}}{\partial \xi_{1}^{4}}= & \rho h\left(1-\chi\left(\xi_{1}\right)\right)+\sum_{l=0}^{j} \frac{\xi_{1}^{l}}{l !} \frac{\partial^{l+2} d_{( \pm) j-\gamma-4-l}^{1}}{\partial t^{2} \partial x_{1}^{l}}(0, t) \\
& +\sum_{l=2}^{j} \frac{\partial^{4}}{\xi_{1}^{4}}\left(\left(1-x\left(\xi_{1}\right)\right) \frac{\xi_{1}^{l}}{l !}\right) \frac{\partial^{l} d_{( \pm) j-l}^{1}}{\partial x_{1}^{l}}(0, t) \\
& +\mu \sum_{l=0}^{j} \frac{\partial^{4}}{\xi_{1}^{4}}\left(\left(1-\chi\left(\xi_{1}\right)\right) \frac{\xi_{1}^{l}}{l !}\right) \frac{\partial^{l+1} d_{( \pm) j-\gamma-l}^{1}}{\partial t \partial x_{1}^{l}}(0, t) \\
& -\left(1-x\left(\xi_{1}\right)\right) \sum_{l=0}^{j} \frac{\xi_{1}^{l}}{l !} \frac{\partial^{l} p_{j-5-l}^{1}}{\partial x_{1}^{l}}(0, \pm 1, t) \\
& -\left(1-\chi\left(\xi_{1}\right)\right) \sum_{l=0}^{j} \frac{\xi_{1}^{l}}{l !} \frac{\partial^{l} q_{j-4-l}^{1}}{\partial x_{1}^{l}}(0, t)
\end{aligned}
$$

$\frac{\partial^{a} \widehat{d}_{( \pm j-1}^{(0)}}{\partial \xi_{1}^{a}} \longrightarrow 0, \quad$ uniformly, when $\xi_{1} \longrightarrow-\infty, a \in\{0,1,2,3\}$

and by $\tilde{d}_{( \pm) j-1}^{(0)}$ the unique solution of

$$
\begin{gathered}
\frac{\partial^{4} \tilde{d}_{( \pm) j-1}^{(0)}}{\partial \xi_{1}^{4}}=\frac{\partial^{4} \widehat{d}_{( \pm) j-1}^{(0)}}{\partial \xi_{1}^{4}}-\rho h \frac{\partial^{2} d_{( \pm) j-\gamma-4}^{(0)}}{\partial t^{2}}-\mu \frac{\partial^{5} d_{( \pm) j-\gamma}^{(0)}}{\partial \xi_{1}^{4} \partial t}+p_{j-5 / \xi_{2}= \pm 1}^{(0)} \\
\frac{\partial^{a} \widetilde{d}_{( \pm) j-1}^{(0)}}{\partial \xi_{1}^{a}} \longrightarrow 0, \quad \text { uniformly, when } \xi_{1} \longrightarrow-\infty, a \in\{0,1,2,3\}
\end{gathered}
$$


Introducing the asymptotic solution into $\left(2.6_{3}\right.$ and identifying the coefficients of the power of $\varepsilon$ we obtain:

$$
\begin{aligned}
d_{( \pm) j}^{(0)}\left(\xi_{1}, \pm 1, t\right)= & \tilde{d}_{( \pm) j-1}^{(0)}\left(\xi_{1}, \pm 1, t\right)-\left(1-\chi\left(\xi_{1}\right)\right) \\
& \times\left(\tilde{d}_{( \pm) j-1}^{(0)}(2, \pm 1, t)-\left(2-\xi_{1}\right) \frac{\partial \tilde{d}_{( \pm) j-1}^{(0)}}{\partial \xi_{1}}(2, \pm 1, t)\right) \xi_{1}>2 \\
d_{( \pm) j}^{1}(0, t)= & 2 \frac{\partial \tilde{d}_{( \pm) j-1}^{(0)}}{\partial \xi_{1}}(2, \pm 1, t)-\tilde{d}_{( \pm) j-1}^{(0)}(2, \pm 1, t), \\
\frac{\partial d_{( \pm) j}^{1}}{\partial x_{1}}(0, t)= & -\frac{\partial \tilde{d}_{( \pm) j}^{(0)}}{\partial \xi_{1}}(2, \pm 1, t) .
\end{aligned}
$$

The relation (4.30) gives the corrector in $x=\mathbf{0}$ for the displacements corresponding to one branch of the flow domain.

We notice that the right-hand side of (4.31) is known, since the problems (4.28) and (4.29) can be solved also if we replace $j-1$ by $j$.

We are now in a position to return to the regular part of the asymptotic solution. For each regular part of the asymptotic solution, we need 9 relations, in order to determine the 9 unknown functions of integration. For the regular part corresponding to $D_{\varepsilon}^{1},(4.11)$ and (4.31) give 8 functions: $d_{( \pm) j}^{1}(0, t),\left(\partial d_{( \pm) j}^{1} / \partial x_{1}\right)(0, t), d_{( \pm) j}^{1}(1, t),\left(\partial d_{( \pm) j}^{1} / \partial x_{1}\right)(1, t)$. It remains undetermined $q_{j}^{1}(0, t)$. We show next that at the $j$ th step this function is already determined at the previous step. For this purpose, we return to the problem (4.23). As usual, we should construct boundary layer functions stabilizing to zero at infinity. As we imposed this condition for the velocity in (4.23) $)_{6,7}$, it is known that the pressure stabilizes to some functions of $t$. At the $j$ th step, we determine $q_{j+1}^{1}(0, t)$ and $q_{j+1}^{2}(0, t)$ from the condition of the exponential decay of $p_{j}^{(0)}$ at infinity. To this end, we consider instead of (4.23) and (4.24), the following problem with known right-hand side for $j \geq-1$ :

$$
\begin{gathered}
-v_{0} \Delta_{\xi \xi} \mathbf{u}_{j}^{(0)}+\nabla_{\xi} \bar{p}_{j}^{(0)}=-\rho_{f} \frac{\partial \mathbf{u}_{j-2}^{(0)}}{\partial t}+\mathbf{F}_{j} \quad \text { in } \boldsymbol{B}, \\
\operatorname{div} \mathbf{u}_{j}^{(0)}=\tilde{\theta}_{j} \quad \operatorname{in} \boldsymbol{B}, \\
\mathbf{u}_{j}^{(0)}=\mathbf{0} \quad \text { on } \hat{A}_{+} B_{+} \cup A_{-} B_{-\prime} \\
\mathbf{u}_{j}^{(0)}\left(\xi_{1}, \pm 1, t\right)=\frac{\partial d_{( \pm) j+2-\gamma}^{(0)}}{\partial t}\left(\xi_{1}, \pm 1, t\right) \mathbf{e}_{2} \quad \text { for } \xi_{1} \geq 2, \\
\mathbf{u}_{j}^{(0)}\left( \pm 1, \xi_{2}, t\right)=\frac{\partial d_{( \pm) j+2-\gamma}^{(0)}}{\partial t}\left( \pm 1, \xi_{2}, t\right) \mathbf{e}_{1} \quad \text { for } \xi_{2} \geq 2, \\
\mathbf{u}_{j}^{(0)} \longrightarrow \mathbf{0}, \quad \bar{p}_{j}^{(0)} \longrightarrow \alpha_{j}^{1}(t) \quad \text { uniformly when } \xi_{1} \longrightarrow \infty, \\
\mathbf{u}_{j}^{(0)} \longrightarrow \mathbf{0}, \quad \bar{p}_{j}^{(0)} \longrightarrow \alpha_{j}^{2}(t) \quad \text { uniformly when } \xi_{2} \longrightarrow \infty
\end{gathered}
$$


with $\alpha_{j}^{1}(t), \alpha_{j}^{2}(t)$ unknown. Supposing for the time being that the function $\tilde{\theta}_{j}$ satisfies the compatibility condition, it follows that (4.32) has a unique solution (the uniqueness for the pressure being understood up to an additive function of $t$ ). This means that the function $\alpha_{j}(t)$, with $\alpha_{j}(t)=\alpha_{j}^{1}(t)-\alpha_{j}^{2}(t)$ is fixed. We define for $j \geq-1$

$$
p_{j}^{(0)}=\bar{p}_{j}^{(0)}+\chi\left(\xi_{1}\right) q_{j+1}^{1}(0, t)+\chi\left(\xi_{2}\right) q_{j+1}^{2}(0, t)
$$

Standard computations show that $\left(\mathbf{u}_{j}^{(0)}, \bar{p}_{j}^{(0)}\right)$ is solution for (4.32) iff $\left(\mathbf{u}_{j}^{(0)}, p_{j}^{(0)}\right)$ satisfies (4.23) or (4.24) for $j=-1$ with $p_{j}^{(0)} \rightarrow \alpha_{j}^{i}(t)+q_{j+1}^{i}(0, t)$ uniformly when $\xi_{i} \rightarrow \infty, i=1,2$. To obtain the desired behavior for $p_{j}^{(0)}$ when $\xi_{i} \rightarrow \infty, i=1,2$, it suffices to take $\alpha_{j}^{i}(t)=-q_{j+1}^{i}(0, t)$.

A first equation for the unknowns $q_{j+1}^{1}(0, t)$ and $q_{j+1}^{2}(0, t)$ is

$$
q_{j+1}^{1}(0, t)-q_{j+1}^{2}(0, t)=\alpha_{j}(t)
$$

with $\alpha_{-1}(t)=0$. The other equation is obtained introducing the asymptotic solution into the compatibility condition (2.11):

$$
\begin{aligned}
\int_{0}^{1}\left(d_{(+) j+1}^{1}\left(x_{1}, t\right)-d_{(-) j+1}^{1}\left(x_{1}, t\right)\right) \mathrm{d} x_{1}+\int_{0}^{1}\left(d_{(+) j+1}^{2}\left(x_{2}, t\right)-d_{(-) j+1}^{2}\left(x_{2}, t\right)\right) \mathrm{d} x_{2} \\
=-\sum_{l=0}^{j}\left(\frac{\partial^{l}\left(d_{(+) j-l}^{1}-d_{(-) j-l}^{1}\right)}{\partial x_{1}^{l}}(0, t)+\frac{\partial^{l}\left(d_{(+) j-l}^{2}-d_{(-) j-l}^{2}\right)}{\partial x_{2}^{l}}(0, t)\right) \int_{0}^{3} \frac{\tau^{l}}{l !}(x(\tau)-1) \mathrm{d} \tau \\
-\int_{-\infty}^{0}\left(d_{(+) j}^{(1)}\left(\xi_{1}, 1, t\right)-d_{(+) j}^{(1)}\left(\xi_{1},-1, t\right)\right) \mathrm{d} \xi_{1}-\int_{-\infty}^{0}\left(d_{(+) j}^{(2)}\left(1, \xi_{2}, t\right)-d_{(+) j}^{(2)}\left(-1, \xi_{2}, t\right)\right) \mathrm{d} \xi_{2} \\
-\int_{2}^{\infty}\left(d_{(+) j}^{(0)}\left(\xi_{1}, 1, t\right)-d_{(+) j}^{(0)}\left(\xi_{1},-1, t\right)\right) \mathrm{d} \xi_{1}-\int_{2}^{\infty}\left(d_{(+) j}^{(0)}\left(1, \xi_{2}, t\right)-d_{(+) j}^{(0)}\left(-1, \xi_{2}, t\right)\right) \mathrm{d} \xi_{2} .
\end{aligned}
$$

The right-hand side of (4.35) is known from the $j$ th approximation. Replacing in the left hand side of $(4.35) d_{( \pm) j+1}^{1}$ given by (4.22) (for $j$ replaced by $j+1$ ) and $d_{( \pm) j+1}^{2}$ given by a similar relation, we are leaded to the second equation for the unknowns $q_{j+1}^{1}(0, t)$ and $q_{j+1}^{2}(0, t)$ :

$$
q_{j+1}^{1}(0, t)+q_{j+1}^{2}(0, t)=\beta_{j}(t),
$$


with $\beta_{j}$ a known function for $j \geq-1$, with

$$
\begin{aligned}
\beta_{-1}(t)= & -6 !\left(\int_{0}^{1} I_{x_{1}}^{-1}(f) \mathrm{d} x_{1}+\int_{0}^{1} I_{x_{2}}^{-1}(f) \mathrm{d} x_{2}\right) \\
& +\frac{3 \times 6 !}{2}\left(\int_{-1}^{1} \psi\left(\xi_{2}, t\right) \mathrm{d} \xi_{2} \int_{0}^{1} I_{x_{1}}^{-1}(v) \mathrm{d} x_{1}+\int_{-1}^{1} \psi\left(\xi_{1}, t\right) \mathrm{d} \xi_{1} \int_{0}^{1} I_{x_{2}}^{-1}(v) \mathrm{d} x_{2}\right) \\
& -\frac{6 !}{2}\left(\int_{0}^{1} I_{x_{1}}^{-4}\left(g_{+}-g_{-}\right) \mathrm{d} x_{1}+\int_{0}^{1} I_{x_{2}}^{-4}\left(g_{+}-g_{-}\right) \mathrm{d} x_{2}\right) \\
& +\frac{6 !}{4}\left(\int_{0}^{1} I_{x_{1}}^{-3}\left(g_{+}-g_{-}\right) \mathrm{d} x_{1}+\int_{0}^{1} I_{x_{2}}^{-3}\left(g_{+}-g_{-}\right) \mathrm{d} x_{2}\right) \\
& -\frac{6 !}{4 !}\left(\int_{0}^{1} I_{x_{1}}^{-2}\left(g_{+}-g_{-}\right) \mathrm{d} x_{1}+\int_{0}^{1} I_{x_{2}}^{-2}\left(g_{+}-g_{-}\right) \mathrm{d} x_{2}\right) .
\end{aligned}
$$

From (4.34) and (4.36) we determine $q_{j+1}^{1}(0, t)$ and $q_{j+1}^{2}(0, t)$, which means that the right-hand side of (4.23) is now known, that allows to solve (4.23), if we show that the problem (4.23) is well posed, that is, the compatibility condition is fulfilled. Moreover, this justifies the previous assertion that $q_{j}^{i}(0, t), i=1,2$ are computed at the $(j-1)$ th step. We can say that the entire $j$ th approximation was obtained for $\gamma>3$ if we prove.

Proposition 4.3. For any $j \in \mathbb{N}$, the function $\widetilde{\theta}_{j}$ satisfies the compatibility condition:

$$
\int_{\bar{B}} \tilde{\theta}_{j}\left(\xi_{1}, \xi_{2}, t\right) d \xi=\int_{\partial B} \mathbf{u}_{j}^{(0)} \cdot \mathbf{n} \mathrm{d} s
$$

Proof. From (4.23) and (4.35) it follows:

$$
\begin{gathered}
\int_{\partial B} \mathbf{u}_{j}^{(0)} \cdot \mathbf{n} \mathrm{d} s=-\frac{\mathrm{d}}{\mathrm{d} t}\left(\int_{-\infty}^{0}\left(d_{(+) j+2-\gamma}^{(1)}\left(\xi_{1}, t\right)-d_{(-) j+2-\gamma}^{(1)}\left(\xi_{1}, t\right)\right) \mathrm{d} \xi_{1}\right. \\
\left.-\int_{-\infty}^{0}\left(d_{(+) j+2-\gamma}^{(2)}\left(\xi_{2}, t\right)-d_{(-) j+2-\gamma}^{(2)}\left(\xi_{2}, t\right)\right) \mathrm{d} \xi_{2}\right) \\
-\frac{\mathrm{d}}{\mathrm{d} t} \sum_{l=0}^{j+2-\gamma}\left(\frac{\partial^{l}\left(d_{(+) j+2-\gamma-l}^{1}-d_{(-) j+2-\gamma-l}^{1}\right)}{\partial x_{1}^{l}}(0, t)\right. \\
\left.+\frac{\partial^{l}\left(d_{(+) j+2-\gamma-l}^{2}-d_{(-) j+2-\gamma-l}^{2}\right)}{\partial x_{2}^{l}}(0, t)\right) \int_{0}^{3} \frac{s^{l}}{l !}(x(s)-1) \mathrm{d} s
\end{gathered}
$$




$$
\begin{aligned}
-\frac{\mathrm{d}}{\mathrm{d} t}( & \int_{0}^{1}\left(d_{(+) j+3-\gamma}^{1}\left(x_{1}, t\right)-d_{(-) j+3-\gamma}^{1}\left(x_{1}, t\right)\right) \mathrm{d} x_{1} \\
& \left.+\int_{0}^{1}\left(d_{(+) j+3-\gamma}^{2}\left(x_{2}, t\right)-d_{(-) j+3-\gamma}^{2}\left(x_{2}, t\right)\right) \mathrm{d} x_{2}\right) .
\end{aligned}
$$

We express next the three terms of the right-hand side of the previous equality with respect to the components of the velocity corresponding to the regular parts of the asymptotic solution.

From (4.9), the corresponding relation for $x_{2}=1$ and (2.9) the first term of the righthand side is equal to

$$
T_{1}=-\int_{-1}^{1} u_{1, j}^{1}\left(1, \xi_{2}, t\right) \mathrm{d} \xi_{2}-\int_{-1}^{1} u_{2, j}^{2}\left(\xi_{1}, 1, t\right) \mathrm{d} \xi_{1}
$$

The third term is expressed by means of $(4.12)_{6}$ and its analogous for $\mathbf{u}_{j}^{2}$ and it is equal to

$$
T_{3}=-\int_{0}^{1}\left(u_{2, j}^{1}\left(x_{1}, 1, t\right)-u_{2, j}^{1}\left(x_{1},-1, t\right)\right) \mathrm{d} x_{1}-\int_{0}^{1}\left(u_{1, j}^{2}\left(1, x_{2}, t\right)-u_{1, j}^{2}\left(-1, x_{2}, t\right)\right) \mathrm{d} x_{2}
$$

Computing now $T_{1}+T_{3}$ and using $(4.12)_{3}$ and the similar one for $\mathbf{u}_{j}^{2}$ we get

$$
T_{1}+T_{3}=-\int_{-1}^{1} u_{1, j}^{1}\left(0, \xi_{2}, t\right) \mathrm{d} \xi_{2}-\int_{-1}^{1} u_{2, j}^{2}\left(\xi_{1}, 0, t\right) \mathrm{d} \xi_{1} .
$$

The second term is given by

$$
\begin{aligned}
T_{2}= & \sum_{l=0}^{j}\left(\frac{\partial^{l}\left(u_{2, j-1-l}^{1}(0,1, t)-u_{2, j-1-l}^{1}(0,-1, t)\right)}{\partial x_{1}^{l}}\right. \\
& \left.+\frac{\partial^{l}\left(u_{1, j-1-l}^{2}(1,0, t)-u_{1, j-1-l}^{2}(-1,0, t)\right)}{\partial x_{2}^{l}}\right) \int_{0}^{3} \frac{s^{l}}{l !}(x(s)-1) \mathrm{d} s \\
= & \sum_{l=0}^{j} \int_{(0,3) \times(-1,1)} \operatorname{div}_{\xi}\left(\left(1-x\left(\xi_{1}\right)\right) \frac{\xi_{1}^{l}}{l !}\left(\frac{\partial^{l} u_{1, j-l}^{1}\left(0, \xi_{2}, t\right)}{\partial x_{1}^{l}} \mathbf{e}_{1}-\frac{\partial^{l} u_{2, j-1-l}^{1}\left(0, \xi_{2}, t\right)}{\partial x_{1}^{l}} \mathbf{e}_{2}\right)\right) \mathrm{d} \xi \\
& +\sum_{l=0}^{j} \int_{(-1,1) \times(0,3)} \operatorname{div}_{\xi}\left(\left(1-x\left(\xi_{2}\right)\right) \frac{\xi_{2}^{l}}{l !}\left(\frac{\partial^{l} u_{1, j-1-l}^{2}\left(\xi_{1}, 0, t\right)}{\partial x_{2}^{l}} \mathbf{e}_{1}-\frac{\partial^{l} u_{2, j-l}^{2}\left(\xi_{2}, 0, t\right)}{\partial x_{2}^{l}} \mathbf{e}_{2}\right)\right) \mathrm{d} \xi \\
& +\int_{-1}^{1} u_{1, j}^{1}\left(0, \xi_{2}, t\right) \mathrm{d} \xi_{2}+\int_{-1}^{1} u_{2, j}^{2}\left(\xi_{1}, 0, t\right) \mathrm{d} \xi_{2} .
\end{aligned}
$$


Finally, computing $T_{1}+T_{2}+T_{3}$, we obtain the following expression of the right-hand side of (4.38):

$$
\begin{aligned}
\int_{\partial \boldsymbol{B}} \mathbf{u}_{j}^{(0)} \cdot \mathbf{n} \mathrm{d} s & \\
= & \sum_{l=0}^{j} \int_{(0,3) \times(-1,1)} \operatorname{div}_{\xi}\left(\left(1-\chi\left(\xi_{1}\right)\right) \frac{\xi_{1}^{l}}{l !}\left(\frac{\partial^{l} u_{1, j-l}^{1}\left(0, \xi_{2}, t\right)}{\partial x_{1}^{l}} \mathbf{e}_{1}-\frac{\partial^{l} u_{2, j-1-l}^{1}\left(0, \xi_{2}, t\right)}{\partial x_{1}^{l}} \mathbf{e}_{2}\right)\right) \mathrm{d} \xi \\
& +\sum_{l=0}^{j} \int_{(-1,1) \times(0,3)} \operatorname{div}_{\xi}\left(\left(1-\chi\left(\xi_{2}\right)\right) \frac{\xi_{2}^{l}}{l !}\left(\frac{\partial^{l} u_{1, j-1-l}^{2}\left(\xi_{1}, 0, t\right)}{\partial x_{2}^{l}} \mathbf{e}_{1}-\frac{\partial^{l} u_{2, j-l}^{2}\left(\xi_{1}, 0, t\right)}{\partial x_{2}^{l}} \mathbf{e}_{2}\right)\right) \mathrm{d} \xi .
\end{aligned}
$$

We compute next the left-hand side of (4.38). Since $\chi\left(\xi_{1}\right)=\chi\left(\xi_{2}\right)=0$ on $ß \cap\left\{\xi_{1}<2, \xi_{2}<2\right\}$, we can write:

$$
\int_{B} \tilde{\theta}_{j}\left(\xi_{1}, \xi_{2}, t\right) \mathrm{d} \xi=\int_{(2,3) \times(-1,1)} \tilde{\theta}_{j}\left(\xi_{1}, \xi_{2}, t\right) \mathrm{d} \xi+\int_{(-1,1) \times(2,3)} \tilde{\theta}_{j}\left(\xi_{1}, \xi_{2}, t\right) \mathrm{d} \xi
$$

We express the first term of the right-hand side of the previous relation taking into account that for $\left(\xi_{1}, \xi_{2}\right) \in(2,3) \times(-1,1)$, we have $\chi\left(\xi_{2}\right)=0$ :

$$
\begin{aligned}
& \int_{(2,3) \times(-1,1)} \tilde{\theta}_{j}\left(\xi_{1}, \xi_{2}, t\right) \mathrm{d} \xi \\
& \quad=\int_{(0,3) \times(-1,1)} \operatorname{div}_{\xi}\left(\left(1-x\left(\xi_{1}\right)\right) \sum_{l=0}^{j} \frac{\xi_{1}^{l}}{l !}\left(\frac{\partial^{l} u_{1, j-l}^{1}}{\partial x_{1} l}\left(0, \xi_{2}, t\right) \mathbf{e}_{1}+\frac{\partial^{l} u_{2, j-1-l}^{1}}{\partial x_{1} l}\left(0, \xi_{2}, t\right) \mathbf{e}_{2}\right)\right) \mathrm{d} \xi
\end{aligned}
$$

For obtaining the above equality we also used the property:

$$
\operatorname{div}_{\xi}\left(\sum_{l=0}^{j} \frac{\xi_{1}^{l}}{l !}\left(\frac{\partial^{l} u_{1, j-l}^{1}}{\partial x_{1} l}\left(0, \xi_{2}, t\right) \mathbf{e}_{1}+\frac{\partial^{l} u_{2, j-1-l}^{1}}{\partial x_{1} l}\left(0, \xi_{2}, t\right) \mathbf{e}_{2}\right)\right)=0
$$

A similar result is obtained for the second term of the right-hand side and, comparing the expression of $\int_{\mathcal{B}} \tilde{\theta}_{j}\left(\xi_{1}, \xi_{2}, t\right) \mathrm{d} \xi$ with the right-hand side of $(4.44)$, the proof is complete.

The last result of this subsection gives the leading term of the regular part of the asymptotic solution corresponding to $D_{\varepsilon}^{1}$. 
Proposition 4.4. For $j=0$ the regular part of the asymptotic solution corresponding to $D_{\varepsilon}^{1}$ is given by

$$
\begin{gathered}
u_{1,0}^{1}\left(x_{1}, \xi_{2}, t\right)=-\frac{3}{2}\left(\int_{-1}^{1} \psi\left(\xi_{2}, t\right) d \xi_{2}\right) N_{1}\left(\xi_{2}\right) \\
u_{2,0}^{1}\left(x_{1}, \xi_{2}, t\right)=0 \\
p_{0}^{1}\left(x_{1}, \xi_{2}, t\right)=0 \\
q_{0}^{1}\left(x_{1}, t\right)=\int_{0}^{x_{1}} f(s, t) d s-\frac{3}{2}\left(\int_{-1}^{1} \psi\left(\xi_{2}, t\right) d \xi_{2}\right) \int_{0}^{x_{1}} v(s) d s+\frac{1}{2} \beta_{-1}(t)
\end{gathered}
$$

with $\beta_{-1}(t)$ defined by (4.37) and

$$
\begin{gathered}
\frac{\partial^{4} d_{( \pm) 0}^{1}}{\partial x_{1}^{4}}=g_{ \pm} \pm q_{0}^{1} \\
d_{( \pm) 0}^{1}(0, t)=d_{( \pm) 0}^{1}(1, t)=\frac{\partial d_{( \pm) 0}^{1}}{\partial x_{1}}(0, t)=\frac{\partial d_{( \pm) 0}^{1}}{\partial x_{1}}(1, t)=0 .
\end{gathered}
$$

\subsubsection{The Solution of the Problems for $\gamma=3$}

Unlike in the previous case, for $\gamma=3$ we cannot solve the problems corresponding to $D_{\varepsilon}^{1}$ and to $D_{\varepsilon}^{2}$ separately. We begin with the problems corresponding to the functions with the index 1. The relations (4.15)-(4.17) are still true. Hence, $p_{j}^{1}$ is also given by (4.17). The difference with respect to the previous case is that now we cannot express the other unknowns only in function of $q_{j}^{1}$, since in the right-hand side of (4.16) $d_{(-) j}^{1}$ is also unknown.

We introduce the notations:

$$
\begin{gathered}
d_{j}^{1}\left(x_{1}, t\right)=d_{(+) j}^{1}\left(x_{1}, t\right)+d_{(-) j}^{1}\left(x_{1}, t\right) \\
\Phi_{j-1}^{1}=\Phi_{(+) j-1}^{1}+\Phi_{(-) j-1}^{1}
\end{gathered}
$$

with $\Phi_{( \pm) j-1}^{1}$ given by $(4.14)_{2}$. The function $d_{j}^{1}$ is obtained as the unique solution of a fourthorder differential problem:

$$
\begin{gathered}
\frac{\partial^{4} d_{j}^{1}}{\partial x_{1}^{4}}=\left(g_{+}+g_{-}\right) \delta_{j 0}+\Phi_{j-1}^{1}, \\
d_{j}^{1}(1, t)=-\left(d_{(+) j}^{(1)}(0, t)+d_{(-) j}^{(1)}(0, t)\right), \\
\frac{\partial d_{j}^{1}}{\partial x_{1}}(1, t)=-\left(\frac{\partial d_{(+) j+1}^{(1)}}{\partial \xi_{1}}(0, t)+\frac{\partial d_{(-) j+1}^{(1)}}{\partial \xi_{1}}(0, t)\right),
\end{gathered}
$$




$$
\begin{gathered}
d_{j}^{1}(0, t)=2\left(\frac{\partial \tilde{d}_{(+) j-1}^{(0)}}{\partial \xi_{1}}(2,1, t)+\frac{\partial \tilde{d}_{(-) j-1}^{(0)}}{\partial \xi_{1}}(2,-1, t)\right)-\left(\tilde{d}_{(+) j-1}^{(0)}(2,1, t)+\tilde{d}_{(-) j-1}^{(0)}(2,-1, t)\right), \\
\frac{\partial d_{j}^{1}}{\partial x_{1}}(0, t)=-\left(\frac{\partial \tilde{d}_{(+) j}^{(0)}}{\partial \xi_{1}}(2,1, t)+\frac{\partial \tilde{d}_{(-) j}^{(0)}}{\partial \xi_{1}}(2,-1, t)\right),
\end{gathered}
$$

the boundary conditions being obtained by means of (4.11) and (4.31). This means that $d_{(-)}^{1}$ can also be expressed via $d_{(+) j}^{1}$. We show that all the unknowns of the regular part of the asymptotic solution can be expressed in function of $d_{(+) j}^{1}$; hence, the regular part of the asymptotic solution corresponding to $D_{\varepsilon}^{1}$ is determined if we obtain and solve a problem for $d_{(+) j}^{1}$. As we cannot obtain all the necessary conditions for $d_{(+) j}^{1}$, we have to consider the problem for the couple of unknowns, $d_{(+) j}^{1}, d_{(+) j}^{2}$.

Theorem 4.5. The approximations of the displacement, $d_{(+) j^{\prime}}^{1} d_{(+) j^{\prime}}^{2}$ are obtained as the unique solution of the following system of two sixth-order parabolic equations:

$$
\begin{gathered}
\frac{\partial d_{(+) j}^{1}}{\partial t}\left(x_{1}, t\right)-\frac{1}{3} \frac{\partial}{\partial x_{1}}\left(\frac{1}{v\left(x_{1}\right)} \frac{\partial^{5} d_{(+) j}^{1}}{\partial x_{1}^{5}}\left(x_{1}, t\right)\right)=G_{j}^{1}\left(x_{1}, t\right)-\frac{\partial H^{1}}{\partial x_{1}}\left(x_{1}, t\right) \delta_{j 0}, \\
\frac{\partial d_{(+) j}^{2}}{\partial t}\left(x_{2}, t\right)-\frac{1}{3} \frac{\partial}{\partial x_{2}}\left(\frac{1}{v\left(x_{2}\right)} \frac{\partial^{5} d_{(+) j}^{2}}{\partial x_{2}^{5}}\left(x_{2}, t\right)\right)=G_{j}^{2}\left(x_{2}, t\right)-\frac{\partial H^{2}}{\partial x_{2}}\left(x_{2}, t\right) \delta_{j 0}, \\
d_{(+) j}^{1}(1, t)=-d_{(+) j}^{(1)}(0, t), \quad \frac{\partial d_{(+) j}^{1}}{\partial x_{1}}(1, t)=-\frac{\partial d_{(+) j+1}^{(1)}}{\partial \xi_{1}}(0, t), \\
d_{(+) j}^{1}(0, t)=2 \frac{\partial \tilde{d}_{(+) j-1}^{(0)}}{\partial \xi_{1}}(2,1, t)-\tilde{d}_{(+) j}^{(0)}(2,1, t), \quad \frac{\partial d_{(+) j}^{1}}{\partial x_{1}}(0, t)=-\frac{\partial \tilde{d}_{(+) j}^{(0)}}{\partial \xi_{1}}(2,1, t), \\
d_{(+) j}^{2}(1, t)=-d_{(+) j}^{(2)}(0, t), \quad \frac{\partial d_{(+) j}^{2}}{\partial x_{2}}(1, t)=-\frac{\partial d_{(+) j+1}^{(2)}}{\partial \xi_{2}}(0, t), \\
d_{(+) j}^{2}(0, t)=2 \frac{\partial \tilde{d}_{(+) j-1}^{(0)}}{\partial \xi_{2}}(1,2, t)-\frac{\tilde{d}_{(+) j}^{(0)}(1,2, t),}{\partial d_{(+) j}^{2}}(0, t)=-\frac{\partial \widetilde{d}_{(+) j}^{(0)}}{\partial \xi_{2}}(1,2, t), \\
\frac{\partial^{5} d_{(+) j}^{1}}{\partial x_{1}^{5}}(1, t)=L_{j}^{1}(t), \\
\frac{\partial^{5} d_{(+) j}^{2}}{\partial x_{2}^{5}}(1, t)=L_{j}^{2}(t), \\
\frac{\partial x_{1}^{4}}{4}(0, t)-\frac{\partial^{4} d_{(+) j}^{2}}{\partial x_{2}^{4}}(0, t)=M_{j}(t),
\end{gathered}
$$


Abstract and Applied Analysis

$$
\begin{gathered}
\frac{\partial^{5} d_{(+) j}^{1}}{\partial x_{1}^{5}}(0, t)+\frac{\partial^{5} d_{(+) j}^{2}}{\partial x_{2}^{5}}(0, t)=N_{j}(t), \\
d_{(+) j}^{1}\left(x_{1}, 0\right)=d_{(+) j}^{2}\left(x_{1}, 0\right)=0,
\end{gathered}
$$

where $H^{i}, G_{j}^{i}, L_{j}^{i}(t), M_{j}(t)$, and $N_{j}(t)$ are known functions, given below.

Proof. The first relation between the unknowns $d_{(+) j}^{1}$ and $q_{j}^{1}$ is given by $(4.12)_{4}$, that is,

$$
\frac{\partial^{4} d_{(+) j}^{1}}{\partial x_{1}^{4}}-q_{j}^{1}=g_{+} \delta_{j 0}+\boldsymbol{\Phi}_{(+) j-1}^{1}
$$

The second one is obtained from (4.16) for $\gamma=3$ and $\xi_{2}=1,(4.12)_{6}$ for $\xi_{2}=1$ and $(4.50)_{1}$ :

$$
2 \frac{\partial d_{(+) j}^{1}}{\partial t}=\frac{\partial d_{j}^{1}}{\partial t}+\frac{2}{3} \frac{\partial}{\partial x_{1}}\left(\frac{1}{v\left(x_{1}\right)}\left(\frac{\partial q_{j}^{1}}{\partial x_{1}}-f \delta_{j 0}\right)\right)-\int_{-1}^{1} D^{-2}\left(\frac{\partial}{\partial x_{1}}\left(\frac{1}{v\left(x_{1}\right)} U_{j-1}^{1}\right) \mathrm{d} \xi_{2}\right.
$$

Eliminating $q_{j}^{1}$ from (4.53) and (4.54) we get $(4.52)_{1}$ with

$$
\begin{gathered}
H^{1}\left(x_{1}, t\right)=\frac{1}{3 v\left(x_{1}\right)}\left(\frac{\partial}{\partial x_{1}} g_{+}+f\right) \\
G_{j}^{1}\left(x_{1}, t\right)=\frac{1}{2}\left(\frac{\partial d_{j}^{1}}{\partial t}-\int_{-1}^{1} D^{-2}\left(\frac{\partial}{\partial x_{1}}\left(\frac{1}{v\left(x_{1}\right)} U_{j-1}^{1}\right)\right) d \xi_{2}\right)-\frac{1}{3} \frac{\partial}{\partial x_{1}}\left(\frac{1}{v\left(x_{1}\right)} \frac{\partial}{\partial x_{1}} \Phi_{(+) j-1}^{1}\right) .
\end{gathered}
$$

As we can see, $H^{1}$ depends only on the data and $G_{j}^{1}$ depends on some functions determined at the previous approximations. Similar expressions are obtained for $H^{2}$ and $G_{j}^{2}$.

Relations (4.52) 3,4 are in fact (4.11) and (4.31), respectively, and the same remark holds for $(4.52)_{5,6}$. We obtain next (4.52) as follows: we derivate (4.53) with respect to $x_{1}$ and we take $x_{1}=1$; we also take $x_{1}=1$ in $(4.15)$ and we eliminate $\left(\partial q_{j}^{1} / \partial x_{1}\right)(1, t)$ from these two relations, by means of (4.9). The right-hand side of $(4.52)$ is

$$
\begin{aligned}
L_{j}^{1}(t)= & \left(\frac{\partial}{\partial x_{1}} g_{+}(1, t)+f(1, t)-\frac{3}{2} v_{0} \int_{-1}^{1} \psi \mathrm{d} \xi_{2}\right) \delta_{j 0}+\frac{3}{2} \int_{-1}^{1} D^{-2}\left(U_{j-1}^{1}\right)\left(1, \xi_{2}, t\right) \mathrm{d} \xi_{2} \\
& +\frac{\partial}{\partial x_{1}} \Phi_{(+) j-1}^{1}-\frac{3 v_{0}}{2} \frac{\mathrm{d}}{\mathrm{d} t} \int_{\infty}^{0}\left(d_{(+) j-1}^{(1)}-d_{(-) j-1}^{(1)}\right)\left(\xi_{1}, t\right) \mathrm{d} \xi_{1}
\end{aligned}
$$


and a similar expression we obtain for $L_{j}^{2}(t)$. Relation (4.52) 9 is a consequence of (4.34) for $j+1$ replaced by $j$, of (4.53) for $x_{1}=0$ and of the corresponding relation for the index 2 . The right hand side of $(4.52)$ is

$$
M_{j}^{1}(0, t)=\alpha_{j-1}(t)+\Phi_{(+) j-1}^{1}(0, t)-\Phi_{(+) j-1}^{2}(0, t)
$$

Finally, (4.52) $)_{10}$ is a consequence of the compatibility condition for (4.32), obtained as follows: (4.25) can be written as

$$
\tilde{\theta}_{j}=\widehat{\theta}_{j-1}-\operatorname{div}_{\xi}\left(\chi\left(\xi_{1}\right) u_{1, j}^{1}\left(\xi_{1}, 0, t\right) \mathbf{e}_{1}+\chi\left(\xi_{2}\right) u_{2, j}^{2}\left(0, \xi_{2}, t\right) \mathbf{e}_{2}\right)
$$

where

$$
\begin{array}{r}
\widehat{\theta}_{j-1}=-\operatorname{div}_{\xi}\left(x\left(\xi_{1}\right) \sum_{l=1}^{j} \frac{\xi_{1}^{l-1}}{(l-1) !}\left(\frac{\xi_{1}}{l} \frac{\partial^{l} u_{1, j-l}^{1}}{\partial x_{1}{ }^{l}}\left(0, \xi_{2}, t\right) \mathbf{e}_{1}+\frac{\partial^{l-1} u_{2, j-l}^{1}}{\partial x_{1} l-1}\left(0, \xi_{2}, t\right) \mathbf{e}_{2}\right)\right. \\
\left.+x\left(\xi_{2}\right) \sum_{l=1}^{j} \frac{\xi_{2}^{l-1}}{(l-1) !}\left(\frac{\partial^{l} u_{1, j-l}^{2}}{\partial x_{2}{ }^{l}}\left(\xi_{1}, 0, t\right) \mathbf{e}_{1}+\frac{\xi_{2}}{l} \frac{\partial^{l} u_{2, j-l}^{2}}{\partial x_{2} l}\left(\xi_{1}, 0, t\right) \mathbf{e}_{2}\right)\right)
\end{array}
$$

from (4.32) we get

$$
\begin{aligned}
\gamma_{j}(t):=\frac{\partial q_{j}^{1}}{\partial x_{1}}(0, t)+\frac{\partial q_{j}^{2}}{\partial x_{2}}(0, t)= & \frac{-3 v_{0}}{2} \int_{B} \widehat{\theta}_{j-1} \\
& +\frac{3 v_{0}}{2} \int_{2}^{\infty}\left(d_{(+) j-1}^{(0)}\left(\xi_{1}, 1, t\right)-d_{(-) j-1}^{(0)}\left(\xi_{1},-1, t\right)\right) d \xi_{1} \\
& +\frac{3 v_{0}}{2} \int_{2}^{\infty}\left(d_{(+) j-1}^{(0)}\left(1, \xi_{2}, t\right)-d_{(-) j-1}^{(0)}\left(-1, \xi_{2}, t\right)\right) \mathrm{d} \xi_{2} \\
& +\frac{3}{2} \int_{-1}^{1} D^{-2}\left(U_{j-1}^{1}\right)\left(0, \xi_{2}, t\right) \mathrm{d} \xi_{2}+\frac{3}{2} \int_{-1}^{1} D^{-2}\left(U_{j-1}^{2}\right)\left(\xi_{1}, 0, t\right) \mathrm{d} \xi_{1} .
\end{aligned}
$$

Next, we derivate (4.53), we take $x_{1}=0$ and we add with the same computation for the index 2. Replacing the previous relation, we obtain $(4.52)_{10}$, with

$$
N_{j}(t)=\gamma_{j}(t)+\frac{\partial}{\partial x_{1}}\left(\boldsymbol{\Phi}_{(+) j-1}(0, t)+\boldsymbol{\Phi}_{(-) j-1}(0, t)\right)
$$

which completes the proof. 
The other components of the regular part of the asymptotic solution corresponding to $D_{\varepsilon}^{1}$ have the following expressions:

$$
\begin{aligned}
d_{(-) j}^{1}\left(x_{1}, t\right)= & d_{j}^{1}\left(x_{1}, t\right)-d_{(+) j}^{1}\left(x_{1}, t\right), \\
q_{j}^{1}\left(x_{1}, t\right)= & \frac{\partial^{4} d_{(+) j}^{1}}{\partial x_{1}^{4}}\left(x_{1}, t\right)-g_{+}\left(x_{1}, t\right) \delta_{j 0}+\Phi_{(+) j-1}^{1}\left(x_{1}, t\right), \\
u_{1, j}^{1}\left(x_{1}, \xi_{2}, t\right)= & \frac{1}{v\left(x_{1}\right)} D^{-2}\left(U_{j-1}^{1}\right)+\frac{1}{v\left(x_{1}\right)}\left(\frac{\partial q_{j}^{1}}{\partial x_{1}}-f \delta_{j 0}\right) N_{1}\left(\xi_{2}\right), \\
u_{2, j}^{1}\left(x_{1}, \xi_{2}, t\right)= & \frac{\partial d_{(-) j-\gamma+3}^{1}}{\partial t}-\frac{\partial}{\partial x_{1}}\left(\frac{1}{v\left(x_{1}\right)}\left(\frac{\partial q_{j}^{1}}{\partial x_{1}}-f \delta_{j 0}\right)\right) N_{2}\left(\xi_{2}\right) \\
& -D^{-1} D^{-2}\left(\frac{\partial}{\partial x_{1}}\left(\frac{1}{v\left(x_{1}\right)} U_{j-1}^{1}\right)\right), \\
p_{j}^{1}= & D^{-1}\left(-\rho_{f} \frac{\partial u_{2, j-3}^{1}}{\partial t}+\frac{\partial}{\partial x_{1}}\left(v\left(x_{1}\right) \frac{\partial u_{2, j-3}^{1}}{\partial x_{1}}\right)\right. \\
& \left.+\frac{\partial}{\partial x_{1}}\left(v\left(x_{1}\right) \frac{\partial u_{1, j-1}^{1}}{\partial \xi_{2}}\right)+2 v\left(x_{1}\right) \frac{\partial^{2} u_{2, j-1}^{1}}{\partial \xi_{2}^{2}}\right) .
\end{aligned}
$$

As in the previous subsection, we discuss the expression of the leading term of the asymptotic solution.

Proposition 4.6. For $j=0$ the regular part of the asymptotic solution corresponding to $D_{\varepsilon}^{1}$ is given by

$$
\begin{gathered}
u_{1,0}^{1}\left(x_{1}, \xi_{2}, t\right)=\frac{1}{v\left(x_{1}\right)}\left(\frac{\partial^{5} d_{(+) 0}^{1}}{\partial x_{1}^{5}}\left(x_{1}, t\right)-\frac{\partial}{\partial x_{1}} g_{+}\left(x_{1}, t\right)-f\left(x_{1}, t\right)\right) N_{1}\left(\xi_{2}\right) \\
u_{2,0}^{1}\left(x_{1}, \xi_{2}, t\right)=-\frac{\partial}{\partial x_{1}}\left(\frac{1}{v\left(x_{1}\right)}\left(\frac{\partial^{5} d_{(+) 0}^{1}}{\partial x_{1}^{5}}\left(x_{1}, t\right)-\frac{\partial}{\partial x_{1}} g_{+}\left(x_{1}, t\right)-f\left(x_{1}, t\right)\right)\right) N_{2}\left(\xi_{2}\right) \\
q_{0}^{1}\left(x_{1}, t\right)=\frac{\partial^{4} d_{(+) 0}^{1}}{\partial x_{1}^{4}}\left(x_{1}, t\right)-g_{+}\left(x_{1}, t\right) \\
p_{0}^{1}\left(x_{1}, \xi_{2}, t\right)=0 \\
d_{(-) 0}^{1}\left(x_{1}, t\right)=d_{0}^{1}\left(x_{1}, t\right)-d_{(+) 0}^{1}\left(x_{1}, t\right)
\end{gathered}
$$

with $d_{0}^{1}, d_{(+) 0}^{1}$ satisfying the problems presented in the proof, (4.64) and (4.65). 
Proof. The relations $(4.63)_{1,2,3,4}$ are consequences of (4.15)-(4.17) and of (4.53) for $j=0$. It remains to show that $d_{0}^{1}, d_{(+) 0^{\prime}}^{1} d_{(+) 0}^{2}$ can be determined as unique solutions of some problems.

For $j=0$ the problem for $d_{0}^{1}$ becomes:

$$
\begin{gathered}
\frac{\partial^{4} d_{0}^{1}}{\partial x_{1}^{4}}\left(x_{1}, t\right)=g_{+}(x, t)+g_{-}\left(x_{1}, t\right), \\
d_{0}^{1}(1, t)=\frac{\partial d_{0}^{1}}{\partial x_{1}}(1, t)=0 \\
d_{0}^{1}(0, t)=\frac{\partial d_{0}^{1}}{\partial x_{1}}(0, t)=0
\end{gathered}
$$

Finally, the problem for $d_{(+) 0^{\prime}}^{1} d_{(+) 0}^{2}$ is the following:

$$
\begin{aligned}
& \frac{\partial d_{(+) 0}^{1}}{\partial t}\left(x_{1}, t\right)-\frac{1}{3} \frac{\partial}{\partial x_{1}}\left(\frac{1}{v\left(x_{1}\right)} \frac{\partial^{5} d_{(+) 0}^{1}}{\partial x_{1}^{5}}\left(x_{1}, t\right)\right)=\frac{1}{2} \frac{\partial d_{0}^{1}}{\partial t}-\frac{1}{3} \frac{\partial}{\partial x_{1}}\left(\frac{1}{v\left(x_{1}\right)}\left(\frac{\partial}{\partial x_{1}} g_{+}+f\right)\right), \\
& \frac{\partial d_{(+) 0}^{2}}{\partial t}\left(x_{2}, t\right)-\frac{1}{3} \frac{\partial}{\partial t}\left(\frac{1}{v\left(x_{2}\right)} \frac{\partial^{5} d_{(+) 0}^{2}}{\partial x_{2}^{5}}\left(x_{2}, t\right)\right)=\frac{1}{2} \frac{\partial d_{0}^{2}}{\partial x_{2}}-\frac{1}{3} \frac{\partial}{\partial x_{2}}\left(\frac{1}{v\left(x_{2}\right)}\left(\frac{\partial}{\partial x_{2}} g_{+}+f\right)\right), \\
& d_{(+) 0}^{1}(1, t)=\frac{\partial d_{(+) 0}^{1}}{\partial x_{1}}(1, t)=d_{(+) 0}^{1}(0, t)=\frac{\partial d_{(+) 0}^{1}}{\partial x_{1}}(0, t)=0, \\
& d_{(+) 0}^{2}(1, t)=\frac{\partial d_{(+) 0}^{2}}{\partial x_{2}}(1, t)=d_{(+) 0}^{2}(0, t)=\frac{\partial d_{(+) 0}^{2}}{\partial x_{2}}(0, t)=0, \\
& \frac{\partial^{5} d_{(+) 0}^{1}}{\partial x_{1}^{5}}(1, t)=-\frac{3 v_{0}}{2} \int_{-1}^{1} \psi\left(\xi_{2}, t\right) \mathrm{d} \xi_{2}+f(1, t)+\frac{\partial}{\partial x_{1}} g_{+}(1, t), \\
& \frac{\partial^{5} d_{(+) 0}^{2}}{\partial x_{2}^{5}}(1, t)=-\frac{3 v_{0}}{2} \int_{-1}^{1} \psi\left(\xi_{1}, t\right) \mathrm{d} \xi_{1}+f(1, t)+\frac{\partial}{\partial x_{2}} g_{+}(1, t), \\
& \frac{\partial^{4} d_{(+) 0}^{1}}{\partial x_{1}^{4}}(0, t)-\frac{\partial^{4} d_{(+) 0}^{2}}{\partial x_{2}^{4}}(0, t)=0, \\
& \frac{\partial^{5} d_{(+) 0}^{1}}{\partial x_{1}^{5}}(0, t)+\frac{\partial^{5} d_{(+) 0}^{2}}{\partial x_{2}^{5}}(0, t)=0, \\
& d_{(+) 0}^{1}\left(x_{1}, 0\right)=d_{(+) 0}^{2}\left(x_{2}, 0\right)=0 .
\end{aligned}
$$

At the end of this subsection we notice that the system (4.52) can be solved also for $j$ replaced by $j+1$ which gives, by means of $(4.53), q_{j+1}^{1}(0, t), q_{j+1}^{2}(0, t)$. Hence, the system (4.23) can be solved, and all the $j$ th approximation is determined for $\gamma=3$. 


\subsubsection{Conclusions}

In the previous two subsections we determined the $j$ th approximation of the asymptotic solution for two different cases: $\gamma>3$ and $\gamma=3$. For $\gamma>3$ we can separate the problems for the two branches of the elastic tube and we obtain the regular part of the asymptotic solution (for $D_{1}^{\varepsilon}$ ) as follows:

(i) the macroscopic variable $q_{j}^{1}$ is the unique solution of the problem:

$$
\begin{gathered}
\frac{\partial}{\partial x_{1}}\left(\frac{1}{v\left(x_{1}\right)}\left(\frac{\partial q_{j}^{1}}{\partial x_{1}}\right)\right)=a_{j}^{q}\left(x_{1}, t\right), \\
\frac{\partial q_{j}^{1}}{\partial x_{1}}(1, t)=b_{j}^{q}(t), \\
q_{j}^{1}(0, t)=c_{j}^{q}(t),
\end{gathered}
$$

with $a_{j}^{q}, b_{j}^{q}, c_{j}^{q}$ known functions;

(ii) the macroscopic variables $d_{( \pm) j}^{1}$ are the unique solutions of the fourth-order differential problems:

$$
\begin{gathered}
\frac{\partial^{4} d_{(+) j}^{1}}{\partial x_{1}^{4}}=a_{j}^{d}\left(x_{1}, t\right), \\
d_{(+) j}^{1}(0, t), \quad d_{(+) j}^{1}(1, t), \quad \frac{\partial d_{(+) j}^{1}}{\partial x_{1}}(0, t), \quad \frac{\partial d_{(+) j}^{1}}{\partial x_{1}}(1, t) \text { given, }
\end{gathered}
$$

and a similar one for $d_{(-) j^{\prime}}^{1}$;

(iii) $u_{1, j}^{1}, u_{2, j}^{1}, p_{j}^{1}$ given by (4.15)-(4.17). The main term of the regular part of the asymptotic solution is presented in (4.48)-(4.49). Unlike in the case $\gamma>3$, for $\gamma=3$ the problems must be solved for the two branches together, since they cannot be uncoupled;

(iv) we first introduce the auxiliary functions $d_{j}^{1}, d_{j}^{2}$, with $d_{j}^{1}$ the unique solution of (4.51); then the regular part of the asymptotic solution is obtained as follows;

(v) the macroscopic variables $d_{(+) j}^{1}, d_{(+) j}^{2}$ are obtained as the unique solution of the sixthorder parabolic system (4.52);

(vi) the macroscopic variables $q_{j}^{1}, q_{j}^{2}$ are given by (4.53) and the similar equation for the index 2;

(vii) $u_{1, j}^{1}, u_{2, j}^{1}, p_{j}^{1}$ given also by (4.15)-(4.17), but we obtain different expressions.

The main term of the regular part of the asymptotic solution is given by (4.63) and (4.65). 


\section{Error Estimates}

In the last section, we estimate the error between the exact solution and the asymptotic one, in order to justify the asymptotic expansion. Introducing the asymptotic solution of order $k$, $\left(\widehat{\mathbf{u}}_{a}^{(k)}, \widehat{p}_{a}^{(k)}, \widehat{d}_{ \pm a}^{(k)}\right)$, into (2.6) we want to obtain for it a problem of the same type as (2.6).

The next computations hold for any integer $r \geq 3$. We begin with the continuity equation satisfied by the asymptotic velocity of order $k$. Standard computations give

$$
\operatorname{div}_{x} \widehat{\mathbf{u}}_{a}^{(k)}=d_{\varepsilon}^{(k)} \text { in } \boldsymbol{B}^{\varepsilon} \times(0, T),
$$

with

$$
d_{\varepsilon}^{(k)}(x, t)= \begin{cases}0 & \text { in } B^{\varepsilon} \cap\left\{x_{1}<\frac{1}{8}\right\} \cup\left\{x_{2}<\frac{1}{8}\right\}, \\ -\eta^{\prime}\left(1-x_{i}\right) \sum_{j=0}^{k} \varepsilon^{j+2} u_{1, j}^{(0)}\left(\frac{x}{\varepsilon}, t\right) & \text { in } B^{\varepsilon} \cap\left\{\frac{1}{8}<x_{i}<\frac{1}{4}\right\}, i=1,2, \\ \eta^{\prime}\left(x_{1}\right) \sum_{j=0}^{k} \varepsilon^{j+2} u_{1, j}^{(1)}\left(\frac{x_{1}-1}{\varepsilon}, \frac{x_{2}}{\varepsilon}, t\right) & \text { in } B^{\varepsilon} \cap\left\{\frac{1}{4}<x_{1}<1\right\}, \\ \eta^{\prime}\left(x_{2}\right) \sum_{j=0}^{k} \varepsilon^{j+2} u_{2, j}^{(2)}\left(\frac{x_{1}}{\varepsilon}, \frac{x_{2}-1}{\varepsilon}, t\right) & \text { in } B^{\varepsilon} \cap\left\{\frac{1}{4}<x_{2}<1\right\} .\end{cases}
$$

We notice that the asymptotic velocity of order $k$ is not a divergence free function.

We present next the equations for the asymptotic solution, which correspond to (2.6) and $(2.6)_{3}$ :

$$
\begin{aligned}
& \rho_{f} \frac{\partial \widehat{\mathbf{u}}_{a}^{(k)}}{\partial t}-2 \operatorname{div}_{x}\left(v(x) D_{x} \widehat{\mathbf{u}}_{a}^{(k)}\right)+\nabla_{x} \widehat{p}_{a}^{(k)}=\mathbf{f}+\mathbf{F}_{\varepsilon}^{(k)} \quad \text { in } \mathcal{B}^{\varepsilon} \times(0, T), \\
& \rho h \frac{\partial^{2} \widehat{d}_{ \pm a}^{(k)}}{\partial t^{2}}+\frac{1}{\varepsilon^{\gamma}} \frac{\partial^{4} \widehat{d}_{ \pm a}^{(k)}}{\partial x_{i}^{4}}+\mu \frac{\partial^{5} \widehat{d}_{ \pm a}^{(k)}}{\partial x_{i}^{4} \partial t}=g_{ \pm} \pm \widehat{p}_{\left.a\right|_{\bar{D}_{\varepsilon} \cap \Gamma_{ \pm}^{\varepsilon}} ^{(k)}}^{(k)}+G_{ \pm \varepsilon}^{(k)} \quad \text { on }\left(\bar{D}_{\varepsilon}^{i} \cap \Gamma_{ \pm}^{\varepsilon}\right) \times(0, T), i=1,2,
\end{aligned}
$$

with $\mathbf{F}_{\varepsilon}^{(k)}, G_{ \pm \varepsilon}^{(k)}$ residual known functions. Since the expressions of these residuals are rather complicated, but not too technical to obtain, let us give below only the estimates for their norms in the corresponding spaces: $\left\|\mathbf{F}_{\varepsilon}^{(k)}\right\|_{L^{2}\left(0, T ;\left(L^{2}\left(\mathcal{B}^{\varepsilon}\right)\right)^{2}\right)}=O\left(\varepsilon^{k+3 / 2}\right)$,

$$
\left\|G_{ \pm \varepsilon}^{(k)}\right\|_{L^{2}\left(\left(\bar{D}_{\varepsilon}^{i} \cap \Gamma_{ \pm}^{\varepsilon}\right) \times(0, T)\right)}=\left\{\begin{array}{l}
O\left(\varepsilon^{3 / 2}\right) \text { for } k \in\{0,1,2,3\}, \\
O\left(\varepsilon^{\min \{k-5 / 2, k+4-\gamma\}}\right) \text { for } k \geq 4 .
\end{array}\right.
$$

These estimates will be used to derive finally the error estimate between the exact and the asymptotic solution. More details concerning these estimates can be found in [2, Theorem 5.1]. 
We derive in the sequel the conditions on the elastic boundaries. Introducing the asymptotic solution of order $k$ into $(2.6)_{11}$ we get

$$
\widehat{\mathbf{u}}_{a}^{(k)} \cdot \mathbf{n}= \pm \frac{\partial \widehat{d}_{ \pm a}^{(k)}}{\partial t} \pm A_{ \pm \varepsilon,}^{k} \quad \widehat{\mathbf{u}}_{a}^{(k)} \cdot \boldsymbol{\tau}=0 \quad \text { on } \Gamma_{ \pm}^{\varepsilon} \times(0, T)
$$

where the expression of $A_{ \pm \varepsilon}^{k}$ on $\Gamma_{ \pm}^{\varepsilon} \cap \partial D_{\varepsilon}^{1}$ is given by:

$$
A_{ \pm \varepsilon}^{k}\left(x_{1}, \pm 1, t\right)=\left\{\begin{array}{c}
-\left(\varepsilon^{k+4} \frac{\partial d_{ \pm k+4-\gamma}^{1}}{\partial t}+\cdots+\varepsilon^{k+\gamma} \frac{\partial d_{ \pm k}^{1}}{\partial t}\right)\left(x_{1}, t\right) \\
-\eta\left(x_{1}\right)\left(\varepsilon^{k+3} \frac{\left.\partial d_{ \pm k+3-\gamma}^{(1)}+\cdots+\varepsilon^{k+\gamma} \frac{\partial d_{ \pm k}^{(1)}}{\partial t}\right)\left(\frac{x_{1}-1}{\varepsilon}, \pm 1, t\right)}{\text { on } \Gamma_{ \pm}^{\varepsilon} \cap\left\{x_{1}>\frac{1}{4}\right\},}\right. \\
-x\left(\frac{x_{1}}{\varepsilon}\right)\left(\varepsilon^{k+4} \frac{\partial d_{ \pm k+4-\gamma}^{1}}{\partial t}+\cdots+\varepsilon^{k+\gamma} \frac{\partial d_{ \pm k}^{1}}{\partial t}\right)\left(x_{1}, t\right) \\
-\eta\left(1-x_{1}\right)\left(\varepsilon^{k+3} \frac{\partial d_{ \pm k+3-\gamma}^{(0)}}{\partial t}+\cdots+\varepsilon^{k+\gamma} \frac{\partial d_{ \pm k}^{(0)}}{\partial t}\right)\left(\frac{x_{1}}{\varepsilon}, \pm 1, t\right) \\
\text { on } \Gamma_{ \pm}^{\varepsilon} \cap\left\{2 \varepsilon<x_{1}<\frac{1}{4}\right\},
\end{array}\right.
$$

and an analogous expression can be written on $\Gamma_{ \pm}^{\varepsilon} \cap \partial D_{\varepsilon}^{2}$. We notice that for $\gamma=3$ the first terms in the above definition are zero.

We complete the problem (5.1), (5.3), (5.5) with the following boundary conditions:

$$
\begin{gathered}
\widehat{\mathbf{u}}_{a}^{(k)}=0, \quad \widehat{d}_{ \pm a}^{(k)}=0 \quad \text { on }\left(\widehat{A}_{+}^{\varepsilon} B_{+}^{\varepsilon} \cup A_{-}^{\varepsilon} B_{-}^{\varepsilon}\right) \times(0, T), \\
\widehat{\mathbf{u}}_{a}^{(k)}\left(1, x_{2}, t\right)=\varepsilon^{2} \psi^{\varepsilon}\left(x_{2}, t\right) \mathbf{e}_{1}+\varepsilon^{k+3} u_{2, k}^{1}\left(1, \xi_{2}, t\right) \mathbf{e}_{2} \quad \text { on } F_{1}^{\varepsilon} \times(0, T), \\
\widehat{\mathbf{u}}_{a}^{(k)}\left(x_{1}, 1, t\right)=\varepsilon^{2} \psi^{\varepsilon}\left(x_{1}, t\right) \mathbf{e}_{2}+\varepsilon^{k+3} u_{1, k}^{2}\left(\xi_{1}, 1, t\right) \mathbf{e}_{1} \quad \text { on } F_{2}^{\varepsilon} \times(0, T), \\
\widehat{d}_{ \pm a}^{(k)}(1, \pm \varepsilon, t)=0, \quad \frac{\partial \widehat{d}_{ \pm a}^{(k)}}{\partial x_{1}}(1, \pm \varepsilon, t)=\varepsilon^{k+\gamma} \frac{\partial d_{ \pm k}^{1}}{\partial x_{1}}(1, t), \\
\widehat{d}_{ \pm a}^{(k)}(2 \varepsilon, \pm \varepsilon, t)=\frac{\partial \widehat{d}_{ \pm a}^{(k)}}{\partial x_{1}}(2 \varepsilon, \pm \varepsilon, t)=0 \\
\widehat{d}_{ \pm a}^{(k)}( \pm \varepsilon, 1, t)=0, \quad \frac{\partial \widehat{d}_{ \pm a}^{(k)}}{\partial x_{1}}( \pm \varepsilon, 1, t)=\varepsilon^{k+\gamma} \frac{\partial d_{ \pm k}^{2}}{\partial x_{1}}(1, t), \\
\widehat{d}_{ \pm a}^{(k)}( \pm \varepsilon, 2 \varepsilon, t)=\frac{\partial \widehat{d}_{ \pm a}^{(k)}}{\partial x_{1}}( \pm \varepsilon, 2 \varepsilon, t)=0 \quad t \in(0, T)
\end{gathered}
$$


and initial conditions:

$$
\begin{gathered}
\widehat{\mathbf{u}}_{a}^{(k)}(x, 0)=0 \quad \text { in } \mathbb{B}^{\varepsilon}, \\
\widehat{d}_{ \pm a}^{(k)}(x, 0)=\frac{\partial \widehat{d}_{ \pm a}^{(k)}}{\partial t}(x, 0)=0 \quad \text { on } \Gamma_{ \pm}^{\varepsilon} .
\end{gathered}
$$

The compatibility condition for the problem (5.1), (5.3) with the boundary and initial conditions (5.5), (5.7), (5.8) is given by:

$$
\begin{aligned}
\int_{B^{\varepsilon}} d_{\varepsilon}^{(k)} \mathrm{d} x= & \int_{2 \varepsilon}^{1} \frac{\partial}{\partial t}\left(\widehat{d}_{+a}^{(k)}\left(x_{1}, \varepsilon, t\right)-\widehat{d}_{-a}^{(k)}\left(x_{1},-\varepsilon, t\right)\right) \mathrm{d} x_{1} \\
& +\int_{2 \varepsilon}^{1} \frac{\partial}{\partial t}\left(\widehat{d}_{+a}^{(k)}\left(\varepsilon, x_{2}, t\right)-\widehat{d}_{-a}^{(k)}\left(-\varepsilon, x_{2}, t\right)\right) \mathrm{d} x_{2} \\
& +\int_{2 \varepsilon}^{1}\left(A_{+\varepsilon}^{k}\left(x_{1}, \varepsilon, t\right)-A_{-\varepsilon}^{k}\left(x_{1},-\varepsilon, t\right)\right) \mathrm{d} x_{1}+\int_{2 \varepsilon}^{1}\left(A_{-\varepsilon}^{k}\left(\varepsilon, x_{2}, t\right)-A_{+\varepsilon}^{k}\left(-\varepsilon, x_{2}, t\right)\right) \mathrm{d} x_{2} .
\end{aligned}
$$

We notice that the boundary conditions for $\widehat{u}_{a}^{(k)}$ and $\widehat{d}_{ \pm a}^{(k)}$ are of different type from those for $\mathbf{u}$ and $d_{ \pm}$, respectively; so we cannot apply directly the estimates (3.20) which hold for different right-hand sides in the equations but for the same boundary conditions. Moreover, the asymptotic velocity of order $k$ is not a divergence free function. To overcome this difficulty, we define new functions $\mathbf{U}_{a}^{(k)}$ and $D_{ \pm a}^{(k)}$ in order to obtain for $\left(\mathbf{U}_{a}^{(k)}, \widehat{p}_{ \pm a}^{(k)}, D_{ \pm a}^{(k)}\right)$ a problem of the same type as (2.6).

\section{Construction of $D_{ \pm a}^{(k)}$}

For this construction we use some ideas from [5]. We first define $D_{ \pm}^{(k)}: F_{ \pm}^{\varepsilon} \times(0, T) \rightarrow \mathbb{R}$, satisfying:

$$
\begin{gathered}
D_{ \pm}^{(k)}(1, \pm \varepsilon, t)=0, \quad \frac{\partial D_{ \pm}^{(k)}}{\partial x_{1}}(1, \pm \varepsilon, t)=\varepsilon^{k+\gamma} \frac{\partial d_{ \pm k}^{1}}{\partial x_{1}}(1, t), \\
D_{ \pm}^{(k)}(2 \varepsilon, \pm \varepsilon, t)=\frac{\partial D_{ \pm}^{(k)}}{\partial x_{1}}(2 \varepsilon, \pm \varepsilon, t)=0, \\
D_{ \pm}^{(k)}( \pm \varepsilon, 1, t)=0, \quad \frac{\partial D_{ \pm}^{(k)}}{\partial x_{1}}( \pm \varepsilon, 1, t)=\varepsilon^{k+\gamma} \frac{\partial d_{ \pm k}^{2}}{\partial x_{2}}(1, t), \\
D_{ \pm}^{(k)}( \pm \varepsilon, 2 \varepsilon, t)=\frac{\partial D_{ \pm}^{(k)}}{\partial x_{2}}( \pm \varepsilon, 2 \varepsilon, t)=0, \\
D_{ \pm}^{(k)}(x, t)=0 \quad\left(x_{1}, x_{2}\right) \in A_{ \pm}^{\widehat{\varepsilon}} B_{ \pm \prime}^{\varepsilon}, \\
D_{ \pm}^{(k)}(x, 0)=\frac{\partial D_{ \pm}^{(k)}}{\partial t}(x, 0)=0,
\end{gathered}
$$




$$
\begin{aligned}
\int_{2 \varepsilon}^{1} \frac{\partial}{\partial t}\left(D_{+}^{(k)}\left(x_{1}, \varepsilon, t\right)\right. & \left.-D_{-}^{(k)}\left(x_{1},-\varepsilon, t\right)\right) \mathrm{d} x_{1}+\int_{2 \varepsilon}^{1} \frac{\partial}{\partial t}\left(D_{+}^{(k)}\left(\varepsilon, x_{2}, t\right)-D_{-}^{(k)}\left(-\varepsilon, x_{2}, t\right)\right) \mathrm{d} x_{2} \\
= & \int_{\mathcal{B}^{\varepsilon}} d_{\varepsilon}^{(k)} \mathrm{d} x-\int_{2 \varepsilon}^{1}\left(A_{+\varepsilon}^{k}\left(x_{1}, \varepsilon, t\right)-A_{-\varepsilon}^{k}\left(x_{1},-\varepsilon, t\right)\right) \mathrm{d} x_{1} \\
& -\int_{2 \varepsilon}^{1}\left(A_{+\varepsilon}^{k}\left(\varepsilon, x_{2}, t\right)-A_{-\varepsilon}^{k}\left(-\varepsilon, x_{2}, t\right)\right) \mathrm{d} x_{2}=: W_{\varepsilon}^{k}(t) .
\end{aligned}
$$

Let us consider

$$
D_{ \pm}^{(k)}(x, t)=\left\{\begin{array}{r}
\left(x_{1}-1\right)\left(x_{1}-2 \varepsilon\right)^{2}\left(a_{ \pm}^{(k) 1}(t)\left(x_{1}-1\right)+\frac{1}{(1-2 \varepsilon)^{2}} \varepsilon^{k+\gamma} \frac{\partial d_{ \pm k}^{1}}{\partial x_{1}}(1, t)\right) \\
\text { in }(2 \varepsilon, 1) \times\{ \pm \varepsilon\} \\
\left(x_{2}-1\right)\left(x_{2}-2 \varepsilon\right)^{2}\left(a_{ \pm}^{(k) 2}(t)\left(x_{2}-1\right)+\frac{1}{(1-2 \varepsilon)^{2}} \varepsilon^{k+\gamma} \frac{\partial d_{ \pm k}^{2}}{\partial x_{2}}(1, t)\right) \\
\text { in }\{ \pm \varepsilon\} \times(2 \varepsilon, 1) \\
0 \quad \text { on } A_{ \pm}^{\widehat{\varepsilon}} B_{ \pm}^{\varepsilon}
\end{array}\right.
$$

with $a_{ \pm}^{(k) i}, i=1,2$ four functions which are determined by imposing some conditions, as we show below. It is obvious that the functions $D_{ \pm}^{(k)}$ satisfy $(5.10)_{1,2,3,4}$ without any conditions on $a_{ \pm}^{(k) i}$.

We introduce next the following notations:

$$
\begin{aligned}
& W_{\varepsilon}^{k, 1}(t)=\int_{D_{\varepsilon}^{1}} d_{\varepsilon}^{(k)} \mathrm{d} x-\int_{2 \varepsilon}^{1}\left(A_{+\varepsilon}^{k}\left(x_{1}, \varepsilon, t\right)-A_{-\varepsilon}^{k}\left(x_{1},-\varepsilon, t\right)\right) \mathrm{d} x_{1} \\
& W_{\varepsilon}^{k, 2}(t)=\int_{D_{\varepsilon}^{2}} d_{\varepsilon}^{(k)} \mathrm{d} x-\int_{2 \varepsilon}^{1}\left(A_{+\varepsilon}^{k}\left(\varepsilon, x_{2}, t\right)-A_{-\varepsilon}^{k}\left(-\varepsilon, x_{2}, t\right)\right) \mathrm{d} x_{2} .
\end{aligned}
$$

We notice that from (5.2) we obtain $W_{\varepsilon}^{k}(t)=W_{\varepsilon}^{k, 1}(t)+W_{\varepsilon}^{k, 2}(t)$. A relation between the functions $a_{+}^{(k) 1}$ and $a_{-}^{(k) 1}$ follows from $(\mathrm{d} / \mathrm{d} t) \int_{2 \varepsilon}^{1}\left(D_{+}^{(k)}\left(x_{1}, \varepsilon, t\right)-D_{-}^{(k)}\left(x_{1},-\varepsilon, t\right)\right) \mathrm{d} x_{1}=W_{\varepsilon}^{k, 1}(t)$, with the initial conditions $(5.10)_{6}$. In a similar way we establish a relation between $a_{+}^{(k) 2}$ and $a_{-}^{(k) 2}$. So we proved the possibility of construction of functions $D_{ \pm}^{(k)}$ which satisfy (5.10) and (5.11).

We define next

$$
\widehat{D}_{ \pm a}^{(k)}=\widehat{d}_{ \pm a}^{(k)}-D_{ \pm}^{(k)}
$$

and we verify that it satisfies the same boundary conditions as $d_{ \pm}$. 


\section{Construction of $\widehat{\mathbf{U}}_{a}^{(k)}$}

We look for a function $\mathbf{U}^{(k)}: \mathbb{B}^{\varepsilon} \rightarrow \mathbb{R}^{2}$ satisfying the following problem, for $t \in(0, T)$ :

$$
\begin{gathered}
\mathbf{U}^{(k)}(t) \in\left(H^{1}\left(\mathcal{B}^{\varepsilon}\right)\right)^{2}, \\
\operatorname{div}_{x} \mathbf{U}^{(k)}(t)=d_{\varepsilon}^{(k)}(t) \quad \text { in } \mathcal{B}^{\varepsilon}, \\
\mathbf{U}^{(k)}(t)=0 \quad \text { in } \mathcal{B}^{\varepsilon} \cap\left\{x_{1}<2 \varepsilon, x_{2}<2 \varepsilon\right\} \\
\mathbf{U}^{(k)}\left(1, x_{2}, t\right)=\varepsilon^{k+3} u_{2, k}^{1}\left(1, \xi_{2}, t\right) \mathbf{e}_{2} \quad \text { on } F_{1}^{\varepsilon}, \\
\mathbf{U}^{(k)}\left(x_{1}, 1, t\right)=\varepsilon^{k+3} u_{1, k}^{2}\left(\xi_{1}, 1, t\right) \mathbf{e}_{1} \quad \text { on } F_{2 \prime}^{\varepsilon} \\
\mathbf{U}^{(k)}\left(x_{1}, \pm \varepsilon, t\right)=\left(\frac{\partial D_{ \pm}^{(k)}}{\partial t} \pm A_{ \pm \varepsilon}^{k}\right)\left(x_{1}, \pm \varepsilon, t\right) \mathbf{e}_{2} \quad \text { on } \Gamma_{ \pm}^{\varepsilon} \cap \partial D_{\varepsilon}^{1} \\
\mathbf{U}^{(k)}\left( \pm \varepsilon, x_{2}, t\right)=\left(\frac{\partial D_{ \pm}^{(k)}}{\partial t} \pm A_{ \pm \varepsilon}^{k}\right)\left( \pm \varepsilon, x_{2}, t\right) \mathbf{e}_{1} \quad \text { on } \Gamma_{ \pm}^{\varepsilon} \cap \partial D_{\varepsilon}^{2} .
\end{gathered}
$$

Remark 5.1. Since $\mathbf{U}^{(k)}$ vanishes in $\boldsymbol{B}^{\varepsilon} \cap\left\{x_{1}<2 \varepsilon, x_{2}<2 \varepsilon\right\}$, (5.15) represents in fact two separate problems, one in $D_{\varepsilon}^{1}$ and the second one on $D_{\varepsilon}^{2}$. The compatibility condition for each problem is fulfilled due to the construction of $D_{ \pm}^{(k)}$.

We can prove that.

Proposition 5.2. The problem (5.15) has at least one solution, with the property

$$
\left\|\mathbf{U}^{(k)}(t)\right\|_{\left(H^{1}\left(\mathcal{B}^{\varepsilon}\right)\right)^{2}}=O\left(\varepsilon^{k+3 / 2}\right) .
$$

Proof. Due to $(5.15)_{3}$, we can consider the problem (5.15) as two separate problems: one in $D_{\varepsilon}^{1}$ and the second in $D_{\varepsilon}^{2}$, each problem with its compatibility condition. So, we can obtain the estimate (5.16) only for $D_{\varepsilon}^{1}$. For any $\left(x_{1}, x_{2}\right) \in D_{\varepsilon}^{1} \cap\left\{x_{1}>2 \varepsilon\right\}$ we introduce the new variable $\left(y_{1}, y_{2}\right)=\left(\left(x_{1}-2 \varepsilon\right) /(1-2 \varepsilon), x_{2} / \varepsilon\right) ;$ obviously $\left(y_{1}, y_{2}\right) \in(0,1) \times(-1,1)$. We define a new function $\boldsymbol{\eta}_{\varepsilon}^{1(k)}:(0,1) \times(-1,1) \times(0, T) \rightarrow \mathbb{R}^{2}$, by $\boldsymbol{\eta}_{\varepsilon}^{1(k)}\left(y_{1}, y_{2}, t\right)=(1 /(1-2 \varepsilon)) U_{1}^{(k)}\left(x_{1}, x_{2}, t\right) \mathbf{e}_{1}+$ $(1 / \varepsilon) U_{2}^{(k)}\left(x_{1}, x_{2}, t\right) \mathbf{e}_{2}$. Obvious computations lead to the following problem for $\boldsymbol{\eta}_{\varepsilon}^{1(k)}(t)$ :

$$
\begin{gathered}
\operatorname{div}_{y} \boldsymbol{\eta}_{\varepsilon}^{1(k)}(t)=d_{\varepsilon}^{(k)}\left((1-2 \varepsilon) y_{1}+2 \varepsilon, \varepsilon y_{2}, t\right) \quad \text { in }(0,1) \times(-1,1), \\
\boldsymbol{\eta}_{\varepsilon}^{1(k)}\left(0, y_{2}, t\right)=\mathbf{0} \quad \text { in }(-1,1), \\
\boldsymbol{\eta}_{\varepsilon}^{1(k)}\left(1, y_{2}, t\right)=\varepsilon^{k+2} u_{2, k}^{1}\left(1, y_{2}, t\right) \mathbf{e}_{2} \quad \text { in }(-1,1), \\
\boldsymbol{\eta}_{\varepsilon}^{1(k)}\left(y_{1}, \pm 1, t\right)=\frac{1}{\varepsilon}\left(\frac{\partial D_{ \pm}^{(k)}}{\partial t} \pm A_{ \pm \varepsilon}^{k}\right)\left((1-2 \varepsilon) y_{1}+2 \varepsilon, \pm \varepsilon, t\right) \mathbf{e}_{2} \quad \text { in }(0,1) .
\end{gathered}
$$


The right-hand sides of $(5.17)_{1,4}$ contain either correctors (which are of order $O(\exp (-\sigma / \varepsilon)$ ), with $\sigma$ independent on $\varepsilon$ ), or terms of order $O\left(\varepsilon^{k+2}\right)$. Applying a result of [15], Chapter III, page 127 we get:

$$
\left\|\boldsymbol{\eta}_{\varepsilon}^{1(k)}(t)\right\|_{\left(H^{1}((0,1) \times(-1,1))\right)^{2}}=O\left(\varepsilon^{k+2}\right) .
$$

Expressing next the norm of $\mathbf{U}^{(k)}$ with respect to the norm of $\boldsymbol{\eta}_{\varepsilon}^{1(k)}$ we obtain $\left\|\mathbf{U}^{(k)}(t)\right\|_{\left(H^{1}\left(D_{\varepsilon}^{1} \cap\left\{x_{1}>2 \varepsilon\right\}\right)\right)^{2}} \leq\left(1 / \varepsilon^{1 / 2}\right)\left\|\boldsymbol{\eta}_{\varepsilon}^{1(k)}(t)\right\|_{\left(H^{1}((0,1) \times(-1,1))\right)^{2}}$, that is, $\left\|\mathbf{U}^{(k)}(t)\right\|_{\left(H^{1}\left(D_{\varepsilon}^{1} \cap\left\{x_{1}>2 \varepsilon\right\}\right)\right)^{2}}=$ $O\left(\varepsilon^{k+(3 / 2)}\right)$. With similar computations we get the same estimate for $\left\|\mathbf{U}^{(k)}(t)\right\|_{\left(H^{1}\left(D_{\varepsilon}^{2} \cap\left\{x_{2}>2 \varepsilon\right\}\right)\right)^{2}}$, which completes the proof.

We notice that from (5.16) and from the regularity of the right-hand sides of (5.17) it follows that $\left\|\mathbf{U}^{(k)}\right\|_{L^{2}\left(0, T ;\left(H^{1}\left(\mathcal{B}^{\varepsilon}\right)\right)^{2}\right)}=O\left(\varepsilon^{k+(3 / 2)}\right)$.

The function

$$
\widehat{\mathbf{U}}_{a}^{(k)}=\widehat{\mathbf{u}}_{a}^{(k)}-\mathbf{U}^{(k)}
$$

satisfies the same type of boundary conditions as $\mathbf{u}$ on $\partial \boldsymbol{B}^{\varepsilon}$. Moreover $\widehat{\mathbf{U}}_{a}^{(k)}(x, 0)=0$, due to $(4.1)_{4}$. As a consequence of the previous constructions, we obtain for $\left(\widehat{\mathbf{U}}_{a}^{(k)}, \widehat{p}_{a}^{(k)}, \widehat{D}_{ \pm a}^{(k)}\right)$ the following problem:

$$
\begin{aligned}
& \rho_{f} \frac{\partial \widehat{\mathbf{U}}_{a}^{(k)}}{\partial t}-2 \operatorname{div}_{x}\left(\mathcal{v}(x) D_{x} \widehat{\mathbf{U}}_{a}^{(k)}\right)+\nabla_{x} \widehat{p}_{a}^{(k)}=\mathbf{f}+\mathcal{F}_{\varepsilon}^{(k)} \quad \text { in } \mathcal{B}^{\varepsilon} \times(0, T), \\
& \operatorname{div}_{x} \widehat{\mathbf{U}}_{a}^{(k)}=0 \quad \text { in } \boldsymbol{B}^{\varepsilon} \times(0, T), \\
& \rho h \frac{\partial^{2} \widehat{D}_{ \pm a}^{(k)}}{\partial t^{2}}+\frac{1}{\varepsilon^{\gamma}} \frac{\partial^{4} \widehat{D}_{ \pm a}^{(k)}}{\partial x_{i}^{4}}+\mu \frac{\partial^{5} \widehat{D}_{ \pm a}^{(k)}}{\partial x_{i}^{4} \partial t}=g_{ \pm} \pm \widehat{p}_{\left.a\right|_{\bar{D}_{\varepsilon}^{i} \cap{ }_{ \pm}^{\varepsilon}} ^{(k)}}^{(k)}+\mathcal{G}_{ \pm \varepsilon}^{(k)} \quad \text { on } \Gamma_{ \pm}^{\varepsilon} \times(0, T), \\
& \widehat{D}_{ \pm a}^{(k)}=0 \quad \text { on } \widehat{A_{ \pm}^{\varepsilon}} B_{ \pm}^{\varepsilon} \times(0, T), \\
& \widehat{\mathbf{U}}_{a}^{(k)}=\boldsymbol{\psi}_{\varepsilon} \quad \text { on }\left(\partial \boldsymbol{B}^{\varepsilon} \backslash\left(\Gamma_{+}^{\varepsilon} \cup \Gamma_{-}^{\varepsilon}\right)\right) \times(0, T), \\
& \widehat{D}_{ \pm a}^{(k)}(1, \pm \varepsilon, t)=\frac{\partial \widehat{D}_{ \pm a}^{(k)}}{\partial x_{1}}(1, \pm \varepsilon, t)=0 \quad \text { in }(0, T), \\
& \widehat{D}_{ \pm a}^{(k)}(2 \varepsilon, \pm \varepsilon, t)=\frac{\partial \widehat{D}_{ \pm a}^{(k)}}{\partial x_{1}}(2 \varepsilon, \pm \varepsilon, t)=0 \quad \text { in }(0, T), \\
& \widehat{D}_{ \pm a}^{(k)}( \pm \varepsilon, 1, t)=\frac{\partial \widehat{D}_{ \pm a}^{(k)}}{\partial x_{2}}( \pm \varepsilon, 1, t)=0 \quad \text { in }(0, T), \\
& \widehat{D}_{ \pm a}^{(k)}( \pm \varepsilon, 2 \varepsilon, t)=\frac{\partial \widehat{D}_{ \pm a}^{(k)}}{\partial x_{2}}( \pm \varepsilon, 2 \varepsilon, t)=0 \quad \text { in }(0, T),
\end{aligned}
$$




$$
\begin{gathered}
\widehat{\mathbf{U}}_{a}^{(k)} \cdot \mathbf{n}= \pm \frac{\partial \widehat{D}_{ \pm a}^{(k)}}{\partial t}, \quad \widehat{\mathbf{U}}_{a}^{(k)} \cdot \boldsymbol{\tau}=0 \quad \text { on } \Gamma_{ \pm}^{\varepsilon} \times(0, T) \\
\widehat{\mathbf{U}}_{a}^{(k)}(x, 0)=\mathbf{0} \quad \text { in } \mathcal{B}^{\varepsilon}, \\
\widehat{D}_{ \pm a}^{(k)}(x, 0)=\frac{\partial \widehat{D}_{ \pm a}^{(k)}}{\partial t}(x, 0)=0 \quad \text { on } \Gamma_{ \pm \prime}^{\varepsilon}
\end{gathered}
$$

with $\mathcal{F}_{\varepsilon}^{(k)}=\mathbf{F}_{\varepsilon}^{(k)}-\rho_{f}\left(\partial \mathbf{U}^{(k)} / \partial t\right)+2 \operatorname{div}_{x}\left(v(x) D_{x} \mathbf{U}^{(k)}\right), \mathcal{G}_{ \pm \varepsilon}^{(k)}=G_{ \pm \varepsilon}^{(k)}-\rho h\left(\partial^{2} D_{ \pm}^{(k)} / \partial t^{2}\right)-\left(1 / \varepsilon^{\gamma}\right)$ $\left(\partial^{4} D_{ \pm}^{(k)} / \partial x_{i}^{4}\right)-\mu\left(\partial^{5} D_{ \pm}^{(k)} / \partial x_{i}^{4} \partial t\right)$.

We are now in a position to apply (3.20) in order to establish the error between the exact solution of (2.6) and its approximation given by (4.4).

Theorem 5.3. Let $\left(\widehat{\mathbf{u}}_{a}^{(k)}, \widehat{p}_{a}^{(k)}, \widehat{d}_{ \pm a}^{(k)}\right)$ be the asymptotic solution of order $k$ given by $(4.4)$ and $(\mathbf{u}, p, d)$ the exact solution of the physical problem (2.6). Then the following estimates hold:

$$
\begin{aligned}
& \left\|\mathbf{u}-\widehat{\mathbf{u}}_{a}^{(k)}\right\|_{L^{\infty}\left(0, T ;\left(L^{2}\left(\mathcal{B}^{\varepsilon}\right)\right)^{2}\right)}=\left\{\begin{array}{l}
O\left(\varepsilon^{3 / 2}\right) \quad \text { for } K \in\{0,1,2,3\}, \\
O\left(\varepsilon^{\min \{k-5 / 2, k+4-\gamma\}}\right) \text { for } K \geq 4,
\end{array}\right. \\
& \left\|D\left(\mathbf{u}-\widehat{\mathbf{u}}_{a}^{(k)}\right)\right\|_{L^{2}\left(0, T ;\left(L^{2}\left(\mathcal{B}^{\varepsilon}\right)\right)^{4}\right)}=\left\{\begin{array}{l}
O\left(\varepsilon^{3 / 2}\right) \quad \text { for } K \in\{0,1,2,3\}, \\
O\left(\varepsilon^{\min \{k-5 / 2, k+4-\gamma\}}\right) \text { for } K \geq 4,
\end{array}\right. \\
& \left\|\frac{\partial}{\partial t}\left(d_{ \pm}-\widehat{d}_{ \pm a}^{(K)}\right)\right\|_{L^{\infty}\left(0, T ; L^{2}\left(\Gamma_{ \pm}^{\varepsilon}\right)\right)}=\left\{\begin{array}{l}
O\left(\varepsilon^{3 / 2}\right) \quad \text { for } K \in\{0,1,2,3\}, \\
O\left(\varepsilon^{\min \{k-5 / 2, k+4-\gamma\}}\right) \text { for } K \geq 4,
\end{array}\right. \\
& \left\|\frac{\partial^{2}}{\partial s^{2}}\left(d_{ \pm}-\widehat{d}_{ \pm a}^{(K)}\right)\right\|_{L^{\infty}\left(0, T ; L^{2}\left(\Gamma_{ \pm}^{\varepsilon}\right)\right)}=\left\{\begin{array}{l}
O\left(\varepsilon^{3 / 2+\gamma / 2}\right) \text { for } K \in\{0,1,2,3\}, \\
O\left(\varepsilon^{\min \{k-5 / 2, k+4-\gamma\}+\gamma / 2}\right) \text { for } K \geq 4,
\end{array}\right. \\
& \left\|\nabla\left(p-\widehat{p}_{a}^{(k)}\right)\right\|_{L^{\infty}\left(0, T ;\left(H^{-1}\left(B^{\varepsilon}\right)\right)^{2}\right)}=\left\{\begin{array}{l}
O\left(\varepsilon^{5 / 2-\gamma / 2}\right) \quad \text { for } K \in\{0,1,2,3\}, \\
O\left(\varepsilon^{\min \{k-5 / 2, k+4-\gamma\}-\gamma / 2}\right) \text { for } K \geq 4 .
\end{array}\right.
\end{aligned}
$$

Proof. Let us obtain the estimate (5.21)2. From (5.16) and (5.19) it follows that

$$
\left\|\mathrm{D}\left(\mathbf{u}-\widehat{\mathbf{u}}_{a}^{(k)}\right)\right\|_{L^{2}\left(0, T ;\left(L^{2}\left(\mathcal{B}^{\varepsilon}\right)\right)^{4}\right)} \leq\left\|\mathrm{D}\left(\mathbf{u}-\widehat{\mathbf{U}}_{a}^{(k)}\right)\right\|_{L^{2}\left(0, T ;\left(L^{2}\left(\mathcal{B}^{\varepsilon}\right)\right)^{4}\right)}+O\left(\varepsilon^{k+3 / 2}\right) .
$$

It remains to estimate the first term of the right-hand side of the previous inequality. For this we first estimate $\min \left(\left\|\Psi_{\varepsilon}^{(k)}\right\|_{L^{2}\left(0, T ;\left(L^{2}\left(\mathcal{B}^{\varepsilon}\right)\right)^{2}\right)},\left\|\mathcal{G}_{ \pm \varepsilon}^{(k)}\right\|_{L^{2}\left(\left(\bar{D}_{\varepsilon}^{i} \cap \Gamma_{ \pm}^{e}\right) \times(0, T)\right)}\right)$ and then we apply (3.20) $)_{2}$. This estimate is given either by $G_{ \pm \varepsilon}^{(k)}$ or by $\left(1 / \varepsilon^{\gamma}\right)\left(\partial^{4} D_{ \pm}^{(k)} / \partial x_{i}^{4}\right)$, the other terms having greater order than these. Following the ideas of [2, Theorem 5.1] we obtain $(5.21)_{2}$; the other estimates of (5.21) are proved with the same technique and the proof is achieved. 
In order to improve the estimates (5.21) we analyze the order of the leading term of the asymptotic solution. We prove that.

Proposition 5.4. For the leading term of the asymptotic solution one has the following estimates:

$$
\begin{gathered}
\left\|\widehat{\mathbf{u}}_{a}^{(0)}\right\|_{L^{\infty}\left(0, T ;\left(L^{2}\left(\mathcal{B}^{\varepsilon}\right)\right)^{2}\right)}=O\left(\varepsilon^{5 / 2}\right), \\
\left\|D \widehat{\mathbf{u}}_{a}^{(0)}\right\|_{L^{2}\left(0, T ;\left(L^{2}\left(\mathcal{B}^{\varepsilon}\right)\right)^{4}\right)}=O\left(\varepsilon^{3 / 2}\right), \\
\left\|\frac{\partial}{\partial t} \widehat{d}_{ \pm a}^{(0)}\right\|_{L^{\infty}\left(0, T ; L^{2}\left(\Gamma_{ \pm}^{\varepsilon}\right)\right)}=O\left(\varepsilon^{\gamma}\right), \\
\left\|\frac{\partial^{2}}{\partial s^{2}} \widehat{d}_{ \pm a}^{(0)}\right\|_{L^{\infty}\left(0, T ; L^{2}\left(\Gamma_{ \pm}^{\varepsilon}\right)\right)}=O\left(\varepsilon^{\gamma}\right), \\
\left\|\nabla \widehat{p}_{a}^{(0)}\right\|_{L^{\infty}\left((0, T) ;\left(H^{-1}\left(\mathcal{B}^{\varepsilon}\right)\right)^{2}\right)}=O\left(\varepsilon^{1 / 2}\right) .
\end{gathered}
$$

Proof. Let us establish (5.23) . From $(4.48)_{1,2}($ for $\gamma>3)$ and from $(4.63)_{1,2}($ for $\gamma=3)$ we obtain for the regular velocity corresponding to $D_{\varepsilon}^{1}$

$$
\left\|\mathbf{u}_{1}^{(0)} X\right\|_{L^{\infty}\left(0, T ;\left(L^{2}\left(B^{\varepsilon}\right)\right)^{2}\right)}=O\left(\varepsilon^{5 / 2}\right) .
$$

This means that the regular part of the asymptotic velocity in the norm of (5.24) is $O\left(\varepsilon^{5 / 2}\right)$. It remains to estimate in the same norm the boundary layer functions. For the corrector corresponding to $x_{1}=1$ we have

$$
\begin{aligned}
\left\|\mathbf{u}_{b l}^{(0) 1} \eta\right\|_{\left(L^{2}\left(\mathcal{B}^{\varepsilon}\right)\right)^{2}} & \leq \varepsilon^{3}\left(\int_{-1 / 4 \varepsilon}^{0} \int_{-1}^{1}\left(\mathbf{u}_{0}^{(1)}\left(\xi_{1}, \xi_{2}, t\right)\right)^{2} \mathrm{~d} \xi\right)^{1 / 2} \\
& \leq \varepsilon^{3}\left(\int_{-\infty}^{0} \int_{-1}^{1}\left(\mathbf{u}_{0}^{(1)}\left(\xi_{1}, \xi_{2}, t\right)\right)^{2} \mathrm{~d} \xi\right)^{1 / 2}=O\left(\varepsilon^{3}\right)
\end{aligned}
$$

which yields

$$
\left\|\mathbf{u}_{b l}^{(0) 1} \eta\right\|_{L^{\infty}\left(0, T ;\left(L^{2}\left(\mathcal{B}^{\varepsilon}\right)\right)^{2}\right)}=O\left(\varepsilon^{3}\right)
$$

Finally, for the corrector in $x=0$ we have

$$
\left\|\mathbf{u}_{b l}^{(0) 0} \eta \cdot \eta\right\|_{\left(L^{2}\left(\mathcal{B}^{\varepsilon}\right)\right)^{2}} \leq \varepsilon^{3}\left(\int_{\bar{B} \cap\left\{\xi_{1}<1 / \varepsilon 4, \xi_{2}<1 / \varepsilon 4\right\}}\left(\mathbf{u}_{0}^{(0)}\left(\xi_{1}, \xi_{2}, t\right)\right)^{2} \mathrm{~d} \xi\right)^{1 / 2}=O\left(\varepsilon^{3}\right)
$$


that leads to an estimate of the same type as (5.26). Hence, we obtain (5.23) as a consequence of (5.24) and (5.26). More details concerning this type of computations can be found in [5, Theorem 5.1].

The previous Proposition allows us to improve the estimates given by Theorem 5.3 in the following sense.

Theorem 5.5. Let $\left(\widehat{\mathbf{u}}_{a}^{(j)}, \widehat{p}_{a}^{(j)}, \widehat{d}_{ \pm a}^{(j)}\right)$ be the asymptotic solution of order $j$ and $(\mathbf{u}, p, d)$ the exact solution of the physical problem. Then the following estimates hold:

$$
\begin{aligned}
& \left\|\mathbf{u}-\widehat{\mathbf{u}}_{a}^{(j)}\right\|_{L^{\infty}\left(0, T ;\left(L^{2}\left(\mathcal{B}^{\varepsilon}\right)\right)^{2}\right)}=O\left(\varepsilon^{j+7 / 2}\right), \\
& \left\|\mathrm{D}\left(\mathbf{u}-\widehat{\mathbf{u}}_{a}^{(j)}\right)\right\|_{L^{2}\left((0, T) ;\left(L^{2}\left(\mathcal{B}^{\varepsilon}\right)\right)^{4}\right)}=O\left(\varepsilon^{j+5 / 2}\right), \\
& \left\|\frac{\partial}{\partial t}\left(d_{ \pm}-\widehat{d}_{ \pm a}^{(j)}\right)\right\|_{L^{\infty}\left(0, T ; L^{2}\left(\Gamma_{ \pm}^{\varepsilon}\right)\right)}=O\left(\varepsilon^{j+\gamma+1}\right), \\
& \left\|\frac{\partial^{2}}{\partial s^{2}}\left(d_{ \pm}-\widehat{d}_{ \pm a}^{(j)}\right)\right\|_{L^{\infty}\left(0, T ; L^{2}\left(\Gamma_{ \pm}^{\varepsilon}\right)\right)}=O\left(\varepsilon^{j+\gamma+1}\right), \\
& \left\|\nabla\left(p-\hat{p}_{a}^{(j)}\right)\right\|_{L^{\infty}\left((0, T) ;\left(H^{-1}\left(\mathcal{B}^{e}\right)\right)^{2}\right)}=O\left(\varepsilon^{j+3 / 2}\right) .
\end{aligned}
$$

Proof. Let $j \geq 0$ be a fixed integer and $k \gg j$. Then from $(5.21)_{1}$ and $(5.23)_{1}$ we get

$$
\begin{aligned}
\left\|\mathbf{u}-\widehat{\mathbf{u}}_{a}^{(j)}\right\|_{L^{\infty}\left(0, T ;\left(L^{2}\left(\mathcal{B}^{\varepsilon}\right)\right)^{2}\right)} & \leq\left\|\mathbf{u}-\widehat{\mathbf{u}}_{a}^{(k)}\right\|_{\left.L^{\infty}\left(0, T ; L^{2}\left(\mathcal{B}^{\varepsilon}\right)\right)^{2}\right)}+\left\|\widehat{\mathbf{u}}_{a}^{(k)}-\widehat{\mathbf{u}}_{a}^{(j)}\right\|_{L^{\infty}\left(0, T ;\left(L^{2}\left(\mathcal{B}^{\varepsilon}\right)\right)^{2}\right)} \\
& =O\left(\varepsilon^{\min \{k-5 / 2, k+4-\gamma\}}\right)+O\left(\varepsilon^{j+7 / 2}\right)=O\left(\varepsilon^{j+7 / 2}\right) .
\end{aligned}
$$

The other estimates of (5.28) are obtained in a similar way, and the proof is achieved.

\section{Acknowledgments}

The authors were partially supported by the following grants: SFR MODMAD of the University of Saint-Etienne, ENISE and the School of Mines of Saint-Etienne, the joint FrenchRussian PICS CNRS grant "Mathematical modeling of blood diseases" and by the Grant no. 14.740.11.0875 "Multiscale problems: analysis and methods" of the Ministry of Education and Research of Russian Federation.

\section{References}

[1] G. P. Panasenko and R. Stavre, "Asymptotic analysis of a periodic flow in a thin channel with viscoelastic wall," Journal de Mathématiques Pures et Appliquées, vol. 85, no. 4, pp. 558-579, 2006.

[2] G. P. Panasenko and R. Stavre, "Asymptotic analysis of a non-periodic flow in a thin channel with visco-elastic wall," Networks and Heterogeneous Media, vol. 3, no. 3, pp. 651-673, 2008.

[3] G. P. Panasenko and R. Stavre, "Asymptotic expansion of the solution to the Stokes flow problem in a thin cylindrical elastic tube," Applied Analysis. In press. 
[4] G. P. Panasenko and R. Stavre, "Well posedness and asymptotic expansion of solution of Stokes equation set in a thin cylindrical elastic tube," in Around the Research of Vladimir Maz'ya. II, A. Laptev, Ed., vol. 12 of International Mathematical Series, Springer, New York, NY, USA, 2010.

[5] G. P. Panasenko and R. Stavre, "Asymptotic analysis of the Stokes flow with variable viscosity in a thin elastic channel," Networks and Heterogeneous Media, vol. 5, no. 4, pp. 783-812, 2010.

[6] G. Cardone, G. P. Panasenko, and Y. Sirakov, "Asymptotic analysis and numerical modeling of mass transport in tubular structures," Mathematical Models $\mathcal{E}$ Methods in Applied Sciences, vol. 20, no. 3, pp. 397-421, 2010.

[7] G. P. Panasenko, Multi-Scale Modelling for Structures and Composites, Springer, Dordrecht, The Netherlands, 2005.

[8] B. M. Haines, I. S. Aranson, L. Berlyand, and D. A. Karpeev, "Effective viscosity of dilute bacterial suspensions: a two-dimensional model," Physical Biology, vol. 5, no. 4, Article ID 046003, pp. 1-9, 2008.

[9] B. Desjardins, M. J. Esteban, C. Grandmont, and P. le Tallec, "Weak solutions for a fluid-elastic structure interaction model," Revista Matemática Complutense, vol. 14, no. 2, pp. 523-538, 2001.

[10] B. Desjardins and M. J. Esteban, "Existence of weak solutions for the motion of rigid bodies in a viscous fluid," Archive for Rational Mechanics and Analysis, vol. 146, no. 1, pp. 59-71, 1999.

[11] C. Grandmont and Y. Maday, "Existence for an unsteady fluid-structure interaction problem," Mathematical Modelling and Numerical Analysis, vol. 34, no. 3, pp. 609-636, 2000.

[12] D. Serre, "Chute libre d'un solide dans un fluide visqueux incompressible. Existence," Japan Journal of Applied Mathematics, vol. 4, no. 1, pp. 99-110, 1987.

[13] R. Temam, Navier-Stokes Equations. Theory and Numerical Analysis, North-Holland, Amsterdam, The Netherlands, 1977.

[14] A. M. Ilyin, Matching of Asymptotic Expansions of Solutions of Boundary Value Problems, Translations of Mathematical Monographs, American Mathematical Society, Washington, DC, USA, 1992.

[15] G. P. Galdi, An Introduction to the Mathematical Theory of the Navier-Stokes Equations, Springer, New York, NY, USA, 1994. 


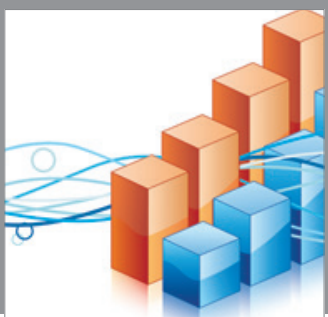

Advances in

Operations Research

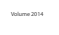

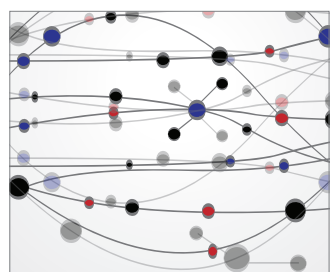

\section{The Scientific} World Journal
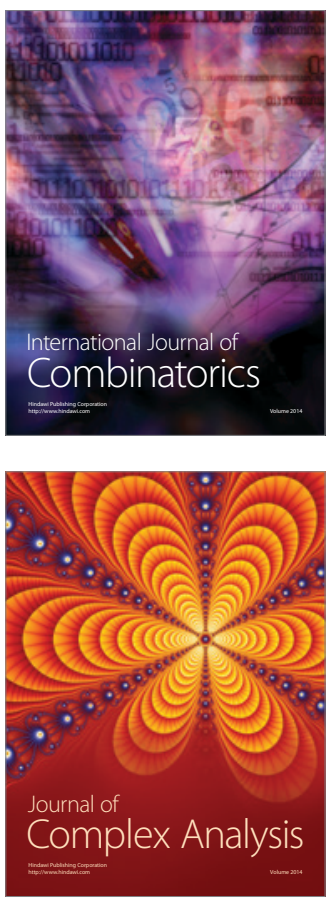

International Journal of

Mathematics and

Mathematical

Sciences
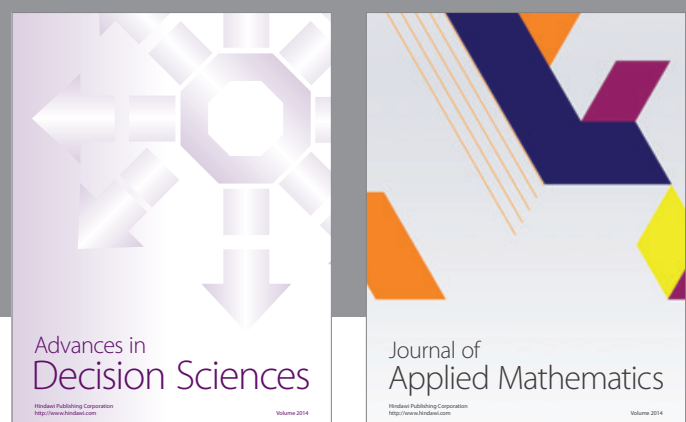

Journal of

Applied Mathematics
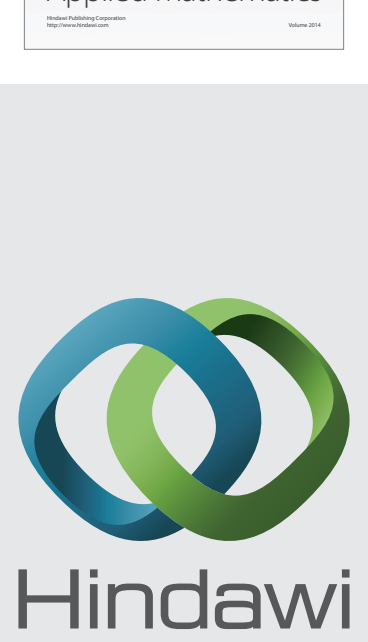

Submit your manuscripts at http://www.hindawi.com
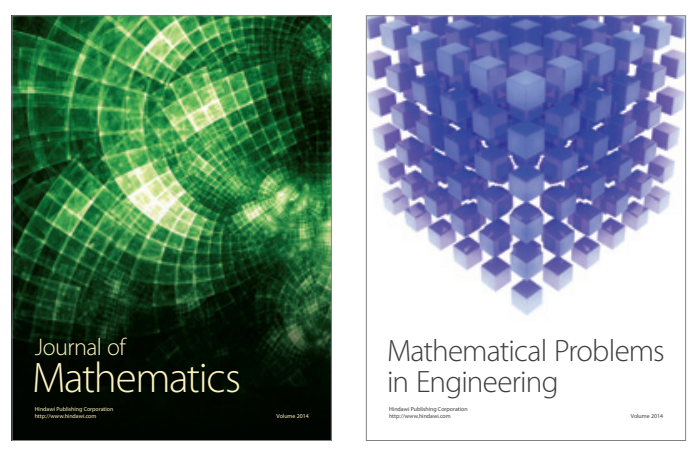

Mathematical Problems in Engineering
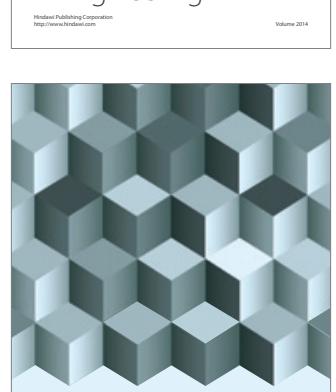

Journal of

Function Spaces
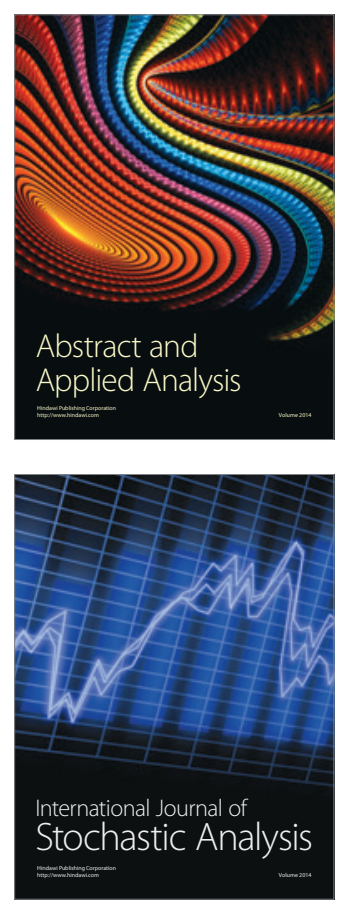

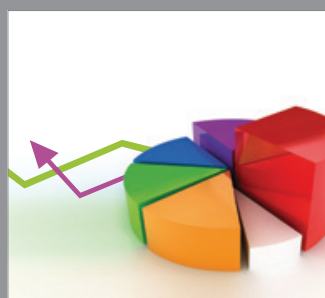

ournal of

Probability and Statistics

Promensencen
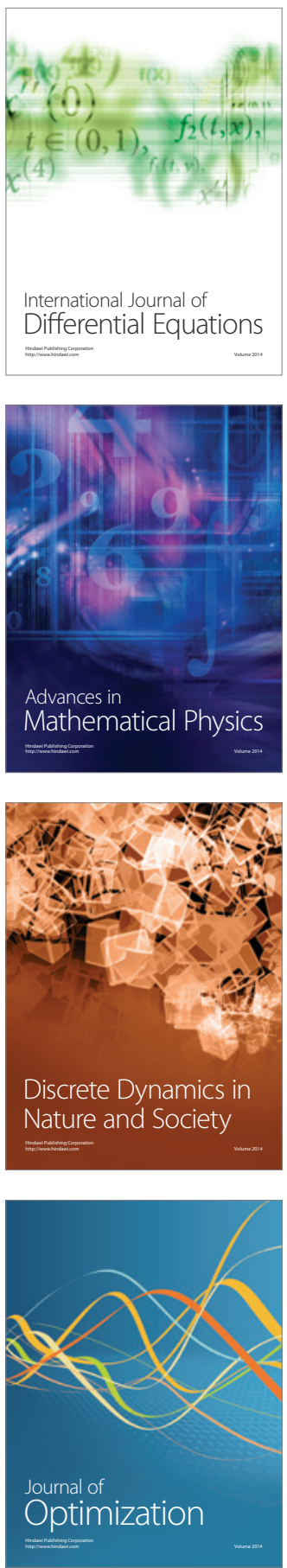